\title{
Solar flares, coronal mass ejections and solar energetic particle event characteristics
}

\author{
Athanasios Papaioannou ${ }^{1, *}$, Ingmar Sandberg ${ }^{1}$, Anastasios Anastasiadis ${ }^{1}$, Athanasios Kouloumvakos ${ }^{2}$, \\ Manolis K. Georgoulis ${ }^{3}$, Kostas Tziotziou ${ }^{1,3}$, Georgia Tsiropoula ${ }^{1}$, Piers Jiggens ${ }^{4}$, and Alain Hilgers ${ }^{4}$ \\ 1 Institute for Astronomy, Astrophysics, Space Applications and Remote Sensing (IAASARS), National Observatory of Athens, \\ I. Metaxa \& Vas. Pavlou St., 15236 Penteli, Greece \\ ${ }^{*}$ Corresponding author: atpapaio@astro.noa.gr \\ 2 Section of Astrogeophysics, Department of Physics, University of Ioannina, 45110 Ioannina, Greece \\ 3 Research Center for Astronomy and Applied Mathematics (RCAAM) of the Academy of Athens, 4 Soranou Efesiou St., \\ 11527 Athens, Greece \\ 4 European Space Research and Technology Centre (ESTEC), Space Environment and Effects Section, Keperlaan 1, \\ 2200AG Noordwijk, The Netherlands
}

Received 17 November 2015 / Accepted 11 October 2016

\begin{abstract}
A new catalogue of 314 solar energetic particle (SEP) events extending over a large time span from 1984 to 2013 has been compiled. The properties as well as the associations of these SEP events with their parent solar sources have been thoroughly examined. The properties of the events include the proton peak integral flux and the fluence for energies above 10, 30, 60 and $100 \mathrm{MeV}$. The associated solar events were parametrized by solar flare (SF) and coronal mass ejection (CME) characteristics, as well as related radio emissions. In particular, for SFs: the soft X-ray (SXR) peak flux, the SXR fluence, the heliographic location, the rise time and the duration were exploited; for CMEs the plane-of-sky velocity as well as the angular width were utilized. For radio emissions, type III, II and IV radio bursts were identified. Furthermore, we utilized element abundances of $\mathrm{Fe}$ and $\mathrm{O}$. We found evidence that most of the SEP events in our catalogue do not conform to a simple two-class paradigm, with the $73 \%$ of them exhibiting both type III and type II radio bursts, and that a continuum of event properties is present. Although, the so-called hybrid or mixed events are found to be present in our catalogue, it was not possible to attribute each SEP event to a mixed/hybrid sub-category. Moreover, it appears that the start of the type III burst most often precedes the maximum of the SF and thus falls within the impulsive phase of the associated SF. At the same time, type III bursts take place within $\approx 5.22$ min, on average, in advance from the time of maximum of the derivative of the SXR flux (Neupert effect). We further performed a statistical analysis and a mapping of the logarithm of the proton peak flux at $E>10 \mathrm{MeV}$, on different pairs of the parent solar source characteristics. This revealed correlations in 3-D space and demonstrated that the gradual SEP events that stem from the central part of the visible solar disk constitute a significant radiation risk. The velocity of the associated CMEs, as well as the SXR peak flux and fluence, are all fairly significantly correlated to both the proton peak flux and the fluence of the SEP events in our catalogue. The strongest correlation to SEP characteristics is manifested by the CME velocity.
\end{abstract}

Key words. Solar flares - Coronal mass ejections - Radio bursts - Solar energetic particles - Element abundances - Neupert effect

\section{Introduction}

Solar energetic particle (SEP) events originate from solar flares (SFs) and/or coronal mass ejections (CMEs). The classical paradigm divides SEP events into two categories: the impulsive and the gradual ones based on their parent solar events (i.e., SFs, CMEs) (Reames 1999, 2013). Impulsive SEP events are considered to be associated with SFs (Anastasiadis 2002; Klein \& Posner 2005), while the gradual ones are considered to be accelerated by CME-driven shocks (Reames 1999; Kahler 2001; Cane \& Lario 2006). Nonetheless, this "two class" picture has proved to be an oversimplification and does not match the diversity and wealth of the observed SEP event properties (Cane et al. 2010). Furthermore, observations have indicated that there is a third category of events, the so-called hybrid or mixed events, during which both SFs and CMEdriven shocks accelerate particles that contribute to large SEP events (Kocharov \& Torsti 2002; Kallenrode 2003). These hybrid or mixed events may look like gradual events but demonstrate properties of impulsive ones (Vainio et al. 2007). Such hybrid SEP events may result from the re-acceleration of remnant ions from previous eruptions by shock waves (Reames 2002) or from the interaction of CMEs (Gopalswamy et al. 2002; Kahler \& Vourlidas 2013). Nevertheless, both the impulsive and gradual SEP events are defined by the magnetic configurations of their parent solar events with the underlying fundamental difference being the acceleration mechanisms: impulsive SEP events stem from resonant stochastic acceleration or magnetic reconnection (Aschwanden 2002), while gradual SEP events result from shock acceleration (Reames 2002).

The most intense SEP events can be observed at a wide range of longitudes. Recent observations by several spacecraft widely distributed in heliolongitude have allowed the observation of SEP events filling a broad region around the Sun (Richardson et al. 2014; Lario et al. 2016). The interpretations offered for such widespread events include: (a) the propagation of CME-driven shocks able to inject SEPs over 
broad angular regions (Rouillard et al. 2012; Lario et al. 2016); (b) the effective propagation of particles perpendicular to the direction of the interplanetary magnetic field (IMF), i.e. cross-field diffusion (Dresing et al. 2012; Dröge et al. 2014); (c) the perpendicular transport of particles through processes other than diffusion (Wiedenbeck et al. 2012). Hence, this wealth of observations from different vantage points within the heliosphere has provided significant insight and further tested the proposed mechanisms for the injection, propagation and acceleration of the SEP events.

A key element for the decoding of the characteristics of SEP events is the establishment of empirical and/or semiempirical statistical relations between their characteristics and the observed properties of the parent solar events (Gopalswamy et al. 2003, 2004; Garcia 2004a, 2004b; Belov et al. 2005; Trottet et al. 2015). Although such relations are not deterministic and thus do not lead to first-principle physical laws, they should be considered as deductive approaches. Therefore, empirical relations attempt to unfold patterns and relationships among the parameters of SEP events and their parent solar events, using all observational evidence at hand, which in turn points to the underlying physical processes of the SEP generation (Balch 2008).

SEP events are one of the most important elements of space weather (Vainio et al. 2009). Given that the complexity of the underlying physical processes of the acceleration and propagation of SEP events is still a very active research area, the prognosis of SEP event occurrence and their corresponding characteristics (e.g., peak flux, duration, fluence) mostly relies on near real-time observations of SFs and CMEs and makes use of the aforementioned empirical relations (Smart \& Shea 1989; Balch 1999; Garcia 2004a, 2004b; Laurenza et al. 2009; Núñez 2011; Papaioannou et al. 2015). In addition, the work from Posner (2007) has proven the concept of short-term forecasting of the appearance and intensity of solar ion events by means of relativistic electrons, making use of the higher speed of these electrons propagating from the Sun to 1 AU. Hence, it appears that catalogues of SEP events - such as the one presented in this work, with updated information concerning the associated parent solar events - constitute an important resource for the studies of SEP events (Vainio et al. 2009; Papaioannou et al. 2014a) and the derivation of empirical relations, which can be foremost used for the forecasting of solar radiation storms.

At this point, it should also be noted that the warning time for the SEP events forecasting, after observation of the parent solar phenomena, is fairly short and cannot be compared to the one that is achieved for the forecasting of solar wind (Rotter et al. 2015) or interplanetary CMEs (Owens \& Cargill 2004) which falls within a timescale of $\approx$ a day(s). This is evident for forecasting systems of SEP events that rely either on remote-sensing observations of the Sun (Laurenza et al. 2009; Núñez 2011; Papaioannou et al. 2015) or on in situ measurements (Posner 2007). In particular, the achieved warning time at a subset of common SEP events between the technique furnished by Laurenza et al. (2009) and the concept of Posner (2007) has shown that the former leads to a median warning time of $55 \mathrm{~min}$ and the latter of $50 \mathrm{~min}$ in advance of the National Oceanic and Atmospheric Administration (NOAA) event threshold crossing (10 pfu at $>10 \mathrm{MeV})$.

In this work, a new catalogue of SEP events observed within a wide range of intensities onboard the Geostationary
Operational Enviromental Satellite (GOES) spacecraft during 1984-2013 has been compiled. This part of our analysis is detailed in Section 2. We have, further, carefully examined the properties and associations of the resulting 314 SEP events with their parent solar events and have established statistical relations between key ingredients of SFs (longitude, maximum soft X-ray (SXR) flux, SXR fluence, rise time, duration), CMEs (velocity, width) and SEP event characteristics (peak flux, fluence). This part of our work is thoroughly presented in Section 3. In addition, Section 4.6.1 presents correlations between the characteristics of SEP events and the parameters of the associated solar events, in an attempt to identify the strongest relationships for use in SEP forecasting. As a next step, Section 4.6.2 proceeds with the mapping of the logarithm of the proton peak flux at $E>10 \mathrm{MeV}$ (a standard SEP metric) over a 2-D space, defined by different pairs of the parameters of the associated solar events to the SEP ones. Finally, the results of our work are summarized and discussed in Section 5.

\section{Data used in the analysis and event associations}

We have built a new comprehensive database that brings together data of SEP events, SFs and CMEs. The database, extending from 1984 to 2013 and covering roughly three solar cycles, is the result of the synthesis of three independent datasets. The procedure for the construction of the database is presented in detail in Sections 2.1-2.3. The database incorporates 314 SEP events - comprising an SEP part, of in situ observations of SEPs, and a "solar" part, of related solar observations, composed by 3693 CMEs-SFs pairs and 20429 SFs. Hence, the database provides a solid basis for the identification of empirical and semi-empirical relations between SEP events and their parent solar event characteristics.

\subsection{Solar data}

Since the drivers of SEP events are SFs and CMEs, the "solar" part of our database is based on two distinct solar datasets; that is, the CME and the SF datasets.

\subsubsection{CME dataset}

The initial CME sample consists of 22143 events observed in the range $2-30$ solar radii $\left(R_{\mathrm{s}}{ }^{1}\right)$ by the Large Angle and Spectrometric Coronagraph (LASCO) (Brueckner et al. 1995) onboard the Solar and Heliospheric Observatory (SOHO) in the period 1996-2013. LASCO carries three telescopes, namely $\mathrm{C} 1, \mathrm{C} 2$ and $\mathrm{C} 3$. However, only $\mathrm{C} 2$ and C3 data are being used since C1 was disabled in June 1998. The CME data utilized in this study are primarily derived by the LASCO CME Catalog at http://cdaw.gsfc.nasa.gov/ CME_list/ (Gopalswamy et al. 2009). The basic CME characteristics employed in our study are:

$-t_{\mathrm{CME}}$ : first appearance of the CME in the $\mathrm{C} 2$ field of view,

$-v_{\mathrm{CME}}$ : mean velocity determined from the linear fit to the height-time measurements,

\footnotetext{
${ }^{1} R_{\mathrm{s}}=695700 \mathrm{~km}$.

2 One should note that the parameters of the LASCO CME catalog are constantly subject to revision; in this study, we have used values which were then current in August 2014.
} 
- $\phi$ : angular width measured at the height beyond which it remains roughly constant and

- $\psi_{\mathrm{CME}}$ : position angle of the CME motion, which is measured from the north pole in the anti-clockwise direction. ${ }^{3}$

For the time period 1984-1995, further efforts were made in order to identify CME events recorded by different instruments (for details see Sect. 2.1.3).

\subsubsection{SFs dataset}

Solar SXR flux measurements, provided by GOES (Unzicker \& Donnelly 1974), recorded in the same period $35306 \mathrm{C}, \mathrm{M}$ and $\mathrm{X}$ class flare events in the 1-8 $\AA$ GOES channel (ftp:// ftp.ngdc.noaa.gov/STP/space-weather/solar-data/solar-features/ solar-flares/X-rays/goes/). From the initial sample of SXR flares we excluded 14818 events for which the location was not available, leading to a sample of $20429 \mathrm{C}, \mathrm{M}$ and X class flares. The following flare characteristics were used in our study:

- $\psi_{\mathrm{F}}$ : position angle of the SXR flare, as inferred by its heliographic coordinates

- $t_{\text {start }}:$ start time of the SXR flare,

$-t_{\mathrm{dec}}$ : fixed decay time of the flare,

$-t_{N}$ : the time of maximum of the derivative of the SXR flux,

$-I_{\mathrm{p}}$ : SXR peak flux of the flare,

- $t_{\mathrm{p}}$ : time of the the SXR peak flux,

$-I_{\text {SXR }}$ : SXR fluence of the flare,

- $I_{10}$ : the SXR flux of the flare, 10 minutes after $t_{\mathrm{p}}$ and

$-I_{N}$ : the maximum of the derivative of the SXR flux that corresponds to $t_{N}$.

For the calculation of $\psi_{\mathrm{F}}$ we used the simplified expression: $\psi_{\mathrm{F}}=\tan ^{-1}[\sin \lambda / \tan \beta]$ using as input the central meridian distance $\lambda$ and the heliographic latitude $\beta$ of the flare; for details, see Youssef (2012).

For the calculation of $I_{\mathrm{SXR}}$ we have applied the following approach: the start of the integration is, naturally, the onset time of the SF, however for the end time we employed a more sophisticated approach by separating SFs into "brief-duration" and "extended-duration". For the cases where the SXR peak flux falls to the $1 / 3$ of its power within 10 minutes ("briefduration", $\left.I_{10} / I_{\mathrm{p}}<1 / 3\right)$, the integration ends at the time when the SXR flux had dropped by one order of magnitude. For the "extended-duration" flares, depending on their $I_{10} / I_{\mathrm{p}}$ ratio, we have used a fixed decay time $\left(t_{\mathrm{dec}}\right)$. In particular, the decay times, presented in Table 2 (supplementary online material) of Laurenza et al. (2009), were utilized. For the cases where $I_{10} / I_{\mathrm{p}}>1.0$, the $t_{\mathrm{dec}}=38 \mathrm{~min} ;$ in case $0.85<I_{10} /$ $I_{\mathrm{p}}<0.90, \quad t_{\mathrm{dec}}=68 \mathrm{~min}$; when $0.90<I_{10} / I_{\mathrm{p}}<0.95$, $t_{\mathrm{dec}}=104 \mathrm{~min}$, and for $0.95<I_{10} / I_{\mathrm{p}}<1.00, t_{\mathrm{dec}}=214 \mathrm{~min}$. Finally, for intermediate cases, where $1 / 3<I_{10} / I_{\mathrm{p}}<0.85$, the integration ends when the SXR peak flux had dropped by $1 / 3$. This approach differs from the one presented in Laurenza et al. (2009) who, instead of the flare start, used as the start time of the integration the $1 / 3$ power point of the rise, under the argument that the SXR maximum is immediately obtained for real-time space weather forecasts and hence, the computed parameters are useful for such purposes. On the other hand,

\footnotetext{
${ }^{3}$ This corresponds to the Measured Position Angle (MPA), included in the SOHO/LASCO CME Catalog.
}

we applied a different criterion since we noticed that in some "brief-duration" cases the integration with the use of the $1 / 3$ rd criterion stopped at an earlier time that was not representative of the overall radio characteristics of these events. In particular, for those "brief-duration" cases the calculated end of the SF was well before the apparent SF end and additionally it was before the end time of the type III radio burst (usually a group of type IIIs). Hence, with the use of the $1 / 3$ rd criterion we would lead to an underestimation of the integration time interval of SXR flux compared to the prolonged integration time for the radio flux. We note that the radio flux start/end integration time does not depend on the SXR maximum, e.g. it is not fixed with respect to the SXR maximum. Despite the fact that our criterion may produce slightly higher fluencies compared to the criterion of Laurenza et al. (2009), with this selection the SXR fluence is more comparable to the radio fluence for the "brief-duration" cases in our sample.

As it is known, observations of SFs in X-rays frequently show that the shape of the rising part of the SXR light curve closely resembles the time integral of the hard X-ray (HXR) light curve. This fact led to the idea that there is a causal relationship between the non-thermal (HXR) and thermal (SXR) emission of a SF, which has become known as the Neupert effect (Neupert 1968; Dennis \& Zarro 1993). In particular, Neupert (1968) proposed the hypothesis that the SXR emission is related to the energy $E$ deposed by non-thermal electrons and that the HXR emission is related to the power $d E / d t$, and correspondingly to the instantaneous number of electrons. Therefore:

$$
F_{\mathrm{HXR}}(t) \propto \frac{\mathrm{d}}{\mathrm{d} t} F_{\mathrm{SXR}}(t)
$$

Hence, the derivative of the SXR flux $\left(F_{\mathrm{SXR}}(t)\right)$ is commonly used as a proxy of the HXR flux $\left(F_{\mathrm{HXR}}(t)\right)$ (Pick \& Vilmer 2008). However, it should be remarked that equation (1) is only an approximation and that the correspondence with theory works only if cooling (by conduction or radiation) is negligible (Benz 2008).

\subsubsection{Associating solar events (CMEs-SFs)}

In order to associate SFs with CMEs we applied both temporal and spatial criteria. Although SFs are being continuously monitored by the solar SXR flux measurements of the GOES from 1976 until today, the unobstructed monitoring of CMEs practically started with the launch of the $\mathrm{SOHO}$ and in particular with the use of LASCO in the end of 1996 with the data flow stabilized in the start of 1997. This leads to a gap of continuous CME data between 1984 and 1997. As a result, we have divided our analysis into two time periods: the SOHO era and the pre-SOHO era.

\section{- SOHO era: 1996-2013}

Temporal criterion: For each CME we used the linear backextrapolation of its trajectory to the surface of the Sun in order to roughly estimate the lift-off time $t_{0}$ (Vršnak et al. 2005). Since CMEs are first observed at C2, there is a time difference $\Delta t$ that stems from the time of the CME's lift-off to its first appearance within the field of view (FOV) of C2. This time difference can be calculated as: $\Delta t=R / v_{\mathrm{CME}}$, assuming that distance $R=3 R_{\mathrm{S}}$ corresponds to the average radial distance of the first measurement in the LASCO C2 FOV 
(Vršnak et al. 2005). Once $t_{0}$ is identified we assume that SFs not occurring within the temporal window $t_{0} \pm 0.06$ days $(0.06$ days $\approx 1.5 \mathrm{~h})$ are most likely not associated with the given CME (Dougherty et al. 2002; Vršnak et al. 2004). In this way, we established a set of 3693 CMEs-SFs pairs whose relative timing was indicative of a causal relationship.

Out of the varying number of SFs that were identified within the temporal window of a CME, we attributed each CME event to a single SF under the so-called "big flare" syndrome (Kahler 1982) but at the same time an attempt was made to pick not only the largest flare, in terms of its maximum magnitude (peak flux) but also the one having the location on the solar disk (longitude) best magnetically connected with the Earth.

Spatial criterion: We then required that the position angle of the flare is within the position angle interval spanned by the CME: $\left|\psi_{\mathrm{CME}}-\psi_{\mathrm{F}}\right|<\phi$.

The combination of both criteria led to the establishment of a sample of 888 CMEs-SFs pairs that fulfilled both criteria (out of which $68 \mathrm{X}, 235 \mathrm{M}$ and $585 \mathrm{C}$ class flares).

\section{- pre-SOHO era: 1984-1995}

An attempt has been made to identify CME events recorded by different instruments and reported in the literature. We have used two primary sources: the catalogue ${ }^{4}$ from the Coronagraph/Polarimeter (C/P) onboard the Solar Maximum Mission (SMM) spacecraft (time span: 1980-1989) ${ }^{5}$ and the white light observations of the solar corona from the Mauna Loa Solar Observatory (MLSO) (time span: 1980-today), which employs three corona meters: MK3, MK4 and recently with the COSMO K-cor. ${ }^{6}$

All of the corona meters at MLSO record CMEs at 1.5-3 $R_{\mathrm{s}}$. SMM C/P reports that the recordings took place up to $4 R_{\mathrm{s}}$. As a result, observations reported in MLSO and SMM, spot CMEs still in the acceleration phase. Furthermore, taking into account that LASCO C2 operates at 2-6 $R_{\mathrm{s}}$, it was not possible to homogenize all datasets (e.g., SMM/C/P; MLSO; SOHO/ LASCO) and make use of their reported characteristics (velocity, width). In addition, we could not apply the spatial criterion to these data. However, given the timing of the observed CMEs an attempt was made to identify whether the reported CMEs were at least temporarily associated with SFs of our catalogue. This led to the identification of 67 CMEs-SFs pairs. Therefore, the reported associations of SFs and CMEs prior to 1996 stem solely from temporal associations.

\subsection{Radio data}

Radio emissions have a rich diagnostic potential about the acceleration and propagation of solar energetic particles (Kouloumvakos et al. 2015). Additionally, they provide important information on the location and temporal evolution of the particle release processes from the corona into the IP medium (Agueda et al. 2014). Moreover, transient solar radio emissions from the Sun indicate electron acceleration, primarily to energies of tens of keVs. Such electrons are likely to be accelerated at any time that high-energy ions are accelerated (Cane et al. 2002). The different types of radio bursts are usually interpreted as signatures of electron beams

\footnotetext{
${ }^{4}$ http://nldr.library.ucar.edu/repository/collections/TECH-NOTE000-000-000-180

5 http://umbra.nascom.nasa.gov/smm/

${ }^{6}$ https://www2.hao.ucar.edu/mlso/mlso-data-and-movies
}

(type IIIs), electrons accelerated in shock waves (type IIs) or as electrons confined in closed-loop structures (type IVs). Of particular interest are type III bursts which are a secondary product of Langmuir waves produced by electron beams and stand as a classic signature of the so-called "impulsive phase" of SFs when the radiation is primarily non-thermal. Such bursts signify the release of electrons into open magnetic field lines (Klein et al. 2005).

In this study, we assembled dynamic radio spectra using data from the WAVES experiment onboard the Wind spacecraft (Bougeret et al. 1995). The Wind/WAVES data derived from http://lep694.gsfc.nasa.gov/waves/data_products.html consist of one-minute resolution radio recordings from $20 \mathrm{kHz}$ to $13.825 \mathrm{MHz}$, from the two WAVES receivers known as RAD1 $(20 \mathrm{kHz}-1.04 \mathrm{MHz})$ and RAD2 (1.075-13.825 MHz). For every SEP event we identified the related type III radio burst based on the duration (start-peak-end time) of the SFs that had been associated with the SEP events - see details in Section 2.4. Additionally, we determined the start and end times of the type III radio emission. In particular, the start of a type III radio burst was defined as the time where the radio emission was above the background level by three standard deviations, preferably at $\sim 10 \mathrm{MHz}$, given that the type III would extend to that frequency. In the cases where the radio emission was partially occulted and was not extended up to $\sim 10 \mathrm{MHz}$ we alternately used $\sim 1 \mathrm{MHz}$ to determine the start of the type III burst. The end time of the type III radio bursts was determined at the time where the radio emission had returned to the pre-event background level by three standard deviations. In cases of complex events in the radio spectra that resulted in ambiguous determination of the type III start and end, we determined those times by visual inspection.

Additionally, for every SEP event in the SOHO era, we identified their related type II radio bursts based on the duration (start-peak-end time) of the SFs that had been associated with the SEP events. Occasionally, we used the available quick-look radio spectrograph plots from http:// secchirh.obspm.fr/ and http://www.sws.bom.gov.au/World Data_Centre/1/9 to identify type II radio bursts lower in the corona. From 2006, onwards, we further used composite radio spectra from STEREO/SWAVES (Bougeret et al. 2008), to achieve better identification of events behind the limb. Finally, we also identified type IV radio bursts, associated with the SEP events of our catalogue.

\subsection{Particle data}

In our analysis, we have used differential proton fluxes from the Energetic Particle Sensor (EPS) - one of the four instruments of the Space Environment Monitor (SEM) - onboard the Geostationary Operational Environmental Satellites (GOES) (Rodriguez et al. 2014). EPS records proton fluxes in seven differential channels (P1-P7) with characteristic energies spanning from a few up to several hundreds of MeVs (Sandberg et al. 2014). In particular, the nominal energy ranges covered by EPS-2 onboard GOES8-12 are: P1: 0.6-4 MeV; P2: 4-9 MeV; P3: $8.7-14.5 \mathrm{MeV}$; P4: 15-40 MeV; P5: 40-80 MeV; P6: $80-165 \mathrm{MeV}$ and P7: $165-500 \mathrm{MeV}$. Furthermore, SEM has been onboard multiple GOES missions ${ }^{7}$ from

\footnotetext{
7 The actual energy ranges may vary from mission to mission. Details on the GOES/EPS energy ranges per mission may be retrieved at http://dev.sepem.oma.be/help/data_pref.html.
} 
GOES05 (1981-1990) to current GOES13 (2010-2016), which implies that the derived continuous dataset spans almost three solar cycles (Onsager et al. 1996; Rodriguez et al. 2014).

At this point it should be noted that the European Space Agency (ESA) Solar Energetic Particle Environment Modelling (SEPEM) (http://dev.sepem.oma.be/) Team (Crosby et al. 2015) has put a lot of effort into the cleaning of the derived datasets from the multiple GOES missions. In practice, all SEP datasets used in the construction of the SEPEM Reference Data Set (RDS) were processed by removing data spikes and correcting (or otherwise removing episodes) where problems occur. GOES/EPS data are used exclusively as the output SEPEM RDS, although the data is cross-calibrated using science-class IMP-8/Goddard Medium Energy Experiment (GME) data. Relatively few gaps and bad points were found in the GOES/EPS data. None of these impacted the peak of an event nor continued for a longer period without there being a back-up EPS instrument which could be used in its place. The short data gaps were filled using a linear interpolation (Crosby et al. 2015) to provide the best estimation of SEP fluences.

In this work, we utilized these cleaned differential proton fluxes from EPS from P2 to P7, made available to us at the time of the study, directly by the SEPEM Team; but currently freely available from ESA through an FTP. ${ }^{8}$

\subsubsection{Identifying SEP events}

The first step of the identification of the SEP events was a threefold procedure:

- First we produced a continuous GOES dataset applying an approach similar to the one used for the implementation of SEPEM, utilizing also the same start/end times, see also Table 1 of Sandberg et al. (2014), for the conjunction of the data from several GOES. In particular, differences in the energy channels between different instruments arose, which were treated so that the data could easily be combined. This required additional processing of the data and merging of the individual datasets without overlaps in time. The best data was selected based on visual inspection, to minimize caveats and favouring more reliable instruments. This procedure was introduced to the GOES/ SEM data by the SEPEM Team (see details on this procedure, here: http://dev.sepem.oma.be/help/data_pref.html) and has led to a continuous proton dataset, which was constructed using GOES/SEM data, covering a time span from 1973 to 2013 (Crosby et al. 2015).

- Next, we re-binned the data point-by-point using a piecewise power law to interpolate between the two closest energies in the raw data. For the re-binning we used the recent-improved re-defined $E_{\text {effective }}$ for each of the P2-P7 GOES energy channels that have been obtained by the cross-calibration of GOES/SEM with IMP-8/GME data (Sandberg et al. 2014, their Tabs. 2 and 3). Therefore, from the six differential energy channels (e.g., P2-P7) made available by the GOES/SEM instrument onboard different versions of the spacecraft throughout its long-lasting operation, we were led to 10 artificial energy channels (F1-F10), as in SEPEM.

\footnotetext{
${ }^{8} \mathrm{ftp}: / / \mathrm{ftp}$. estec.esa.int/private/pjiggens/anonymous/SEPEM_RDS_ v2-00.zip
}

- Finally, we have developed a code and applied an automated procedure to the whole unified re-binned GOES dataset at the energy range $7.23-10.46 \mathrm{MeV}$ (e.g., the respective F2 of the SEPEM energy channels), in order to create an SEP event catalogue comparable to SEPEM.

The code used the following threshold parameters for the determination of SEP enhancements: (a) the differential flux value above which a possible enhancement was marked, (b) the minimum peak flux (intensity threshold to surpass) of the candidate event, (c) the waiting time between two consecutive candidate events and (d) the minimum event duration. A threshold of 0.01 particles $/ \mathrm{cm}^{2} \mathrm{sr} \mathrm{s} \mathrm{MeV} \mathrm{was} \mathrm{exceeded} \mathrm{by}$ 62919 candidate events; a minimum peak of 0.5 pfu led to 345 events exceeding this limit, while setting both the waiting time between events and the duration of the event at $2 \mathrm{~h}$ our sample led to a total number of 291 candidate SEP events. Hence, the automated code returned the time blocks of 291 possible SEP enhancements. The criteria that were set through our code differ from the criteria that were introduced by the SEPEM Team for the implementation of the SEPEM reference event list ${ }^{9}$ which was created for the purpose of generating models for the specification of the SEP enviroment and not for forecasting.

In the second step of our identification, we utilized the differential GOES proton fluxes from 4 to $500 \mathrm{MeV}$, i.e., P2-P7. The candidate SEP enhancements obtained at the previous step resulted in many compound structures that clearly included more than one SEP event. Therefore, we performed a manual separation of these candidate enhancements. This procedure resulted in 390 candidate SEP events. Finally, we filtered out enhancements that we were unable to further analyse which could endanger our statistics (e.g., cases that were registered only at $\mathrm{P} 2$ and/or were within background fluctuations). As a result, we obtained a total of 314 well-defined SEP events. For each of the 314 SEP events we used the differential GOES/SEM proton data (P2-P7) and calculated the onset times per event and per channel using the so-called $\sigma$ method; for details, see Papaioannou et al. (2014b). Figure 1 exemplifies how the manual separation was performed for the SEP events. Figure 1A presents the outcome of the automated procedure for the identification of SEP events for the October 2003 period. Figures 1B-1F present the outputs of the manual break. Vertical lines mark the start and end times of the event as this was identified in this work; titles in each sub-panels provide the number of the event as well as the start and end times; the ordinate in all panels presents the proton intensities (in particles $/ \mathrm{cm}^{2} \mathrm{sr} \mathrm{s} \mathrm{MeV}$ ) and the abscissa presents the time in hours from the onset time of the SEP event, the latter labelled with the first vertical line in each sub-panel. The time resolution of the data is $5 \mathrm{~min}$.

\subsubsection{Calculation of the SEP peak flux and fuence}

For the calculation of the proton integral fluxes we integrate over the differential flux spectra using the following method. First, we re-bin the SEPEM RDS differential spectra using a piecewise power law interpolation scheme in a numerical grid that has 100 bins - logarithmically spaced - within the energy range of $5-500 \mathrm{MeV}$. Then, we perform a numerical integration and calculate the integral flux at the selected energies, $E>10,30,60$ and $100 \mathrm{MeV}$. The calculation of the proton peak flux and fluence values for each event becomes

\footnotetext{
${ }^{9}$ http://dev.sepem.oma.be/help/event_ref.html
} 
(A)

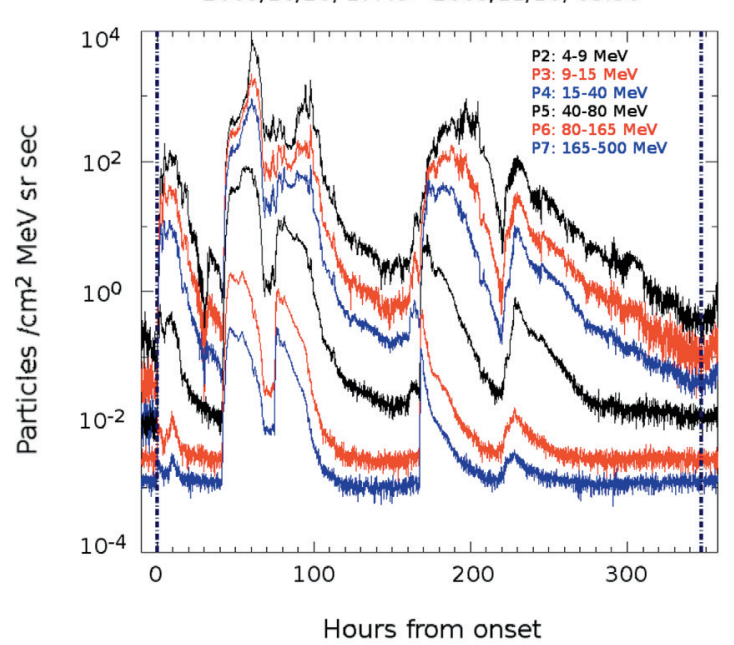

(C)

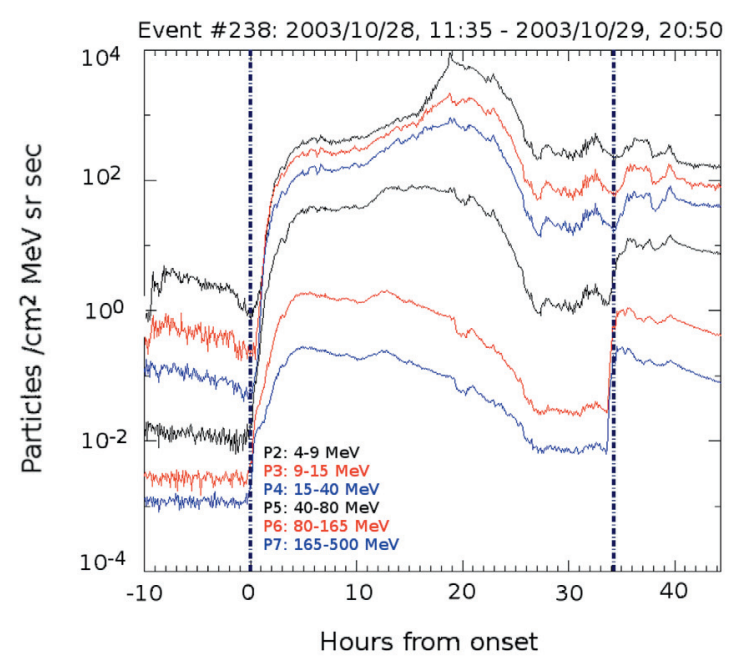

(E)

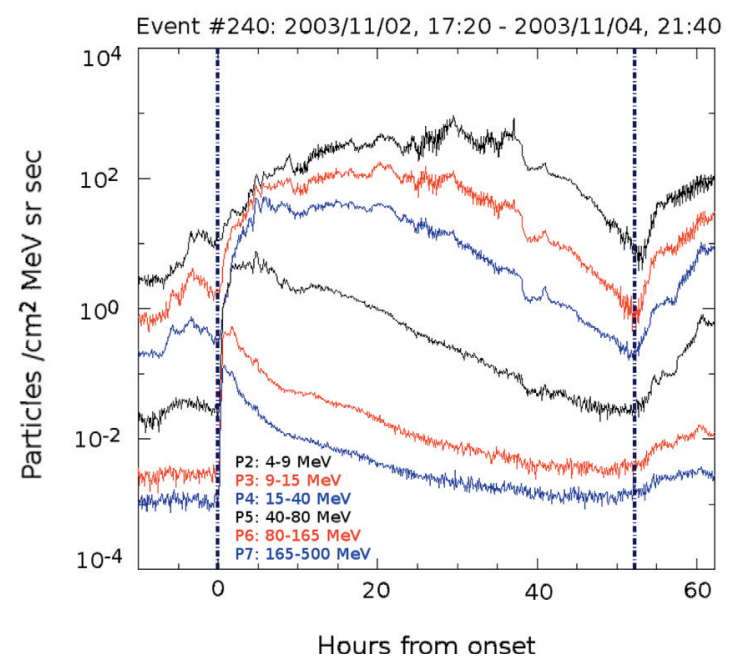

(B)

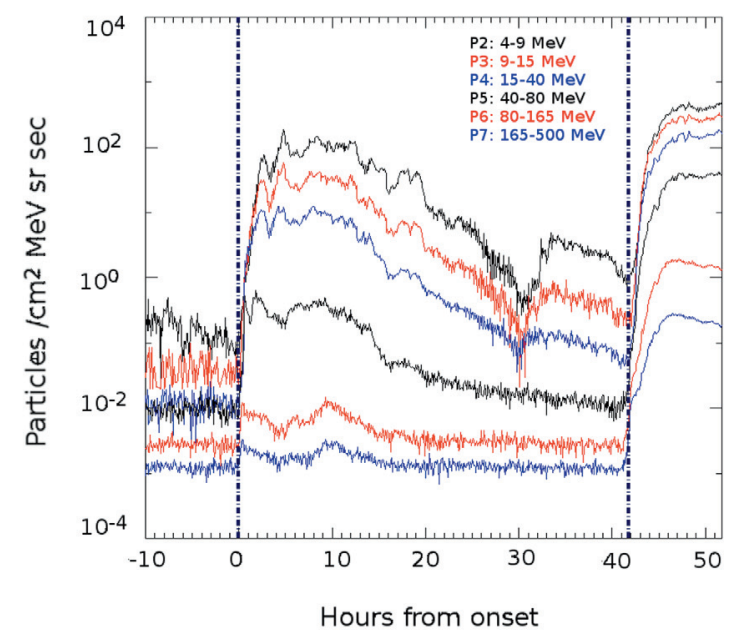

(D)

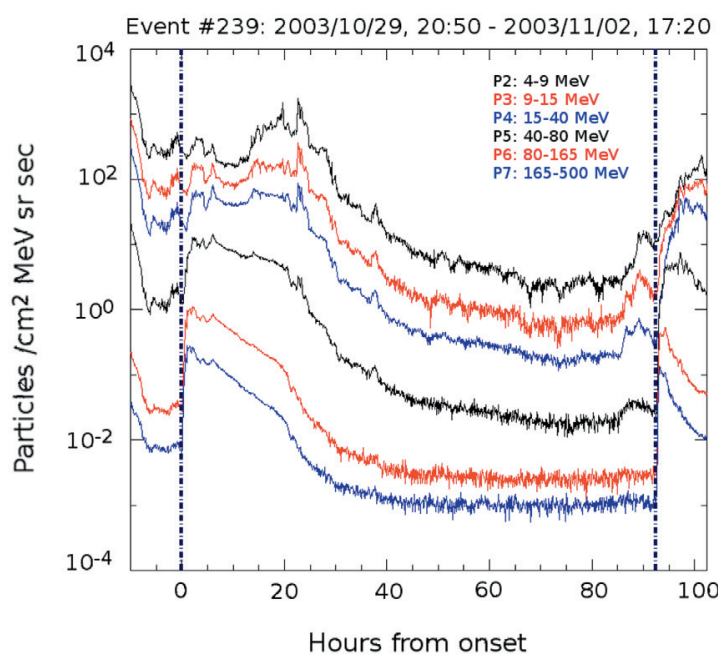

$(\mathrm{F})$

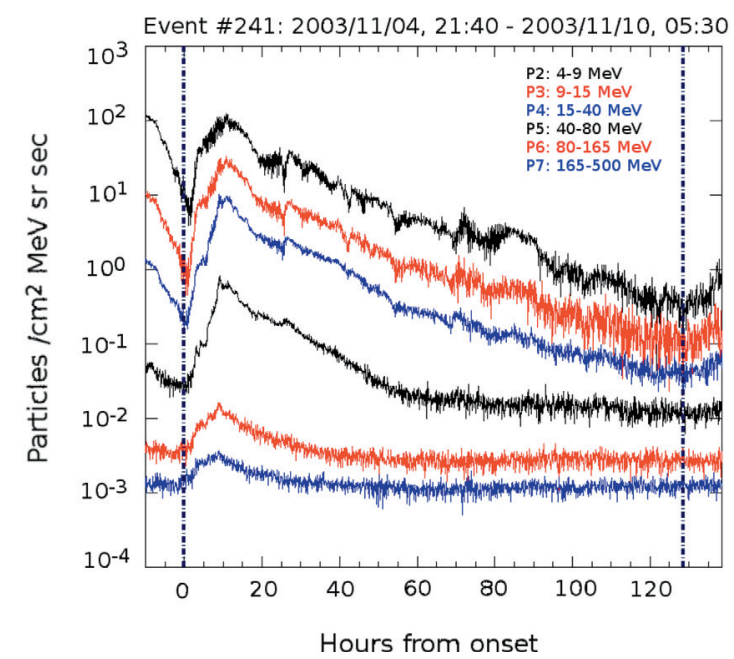

Fig. 1. An illustration of the work scheme for the identification of the SEP events. We present particle recordings, reported onboard GOES/EPS at a wide energy range from 4-500 MeV (i.e. P2-P7; see legends in each panel for details). (A) The outcome of the automated procedure for the identification of SEP events for the October 2003 period. (B-F) The outputs of the manual break. Vertical lines mark the start and end time of the event as identified in this work; titles in each sub-panels provide the number of the event as well the start and end time; the ordinate in all panels presents the proton intensities (in particles/ $\mathrm{cm}^{2} \mathrm{sr} \mathrm{sec} \mathrm{MeV)} \mathrm{and} \mathrm{the} \mathrm{abscissa} \mathrm{presents} \mathrm{the} \mathrm{time} \mathrm{in} \mathrm{hours} \mathrm{from} \mathrm{the} \mathrm{onset} \mathrm{time,} \mathrm{the} \mathrm{latter}$ labelled with the first vertical line in each sub-panel. 

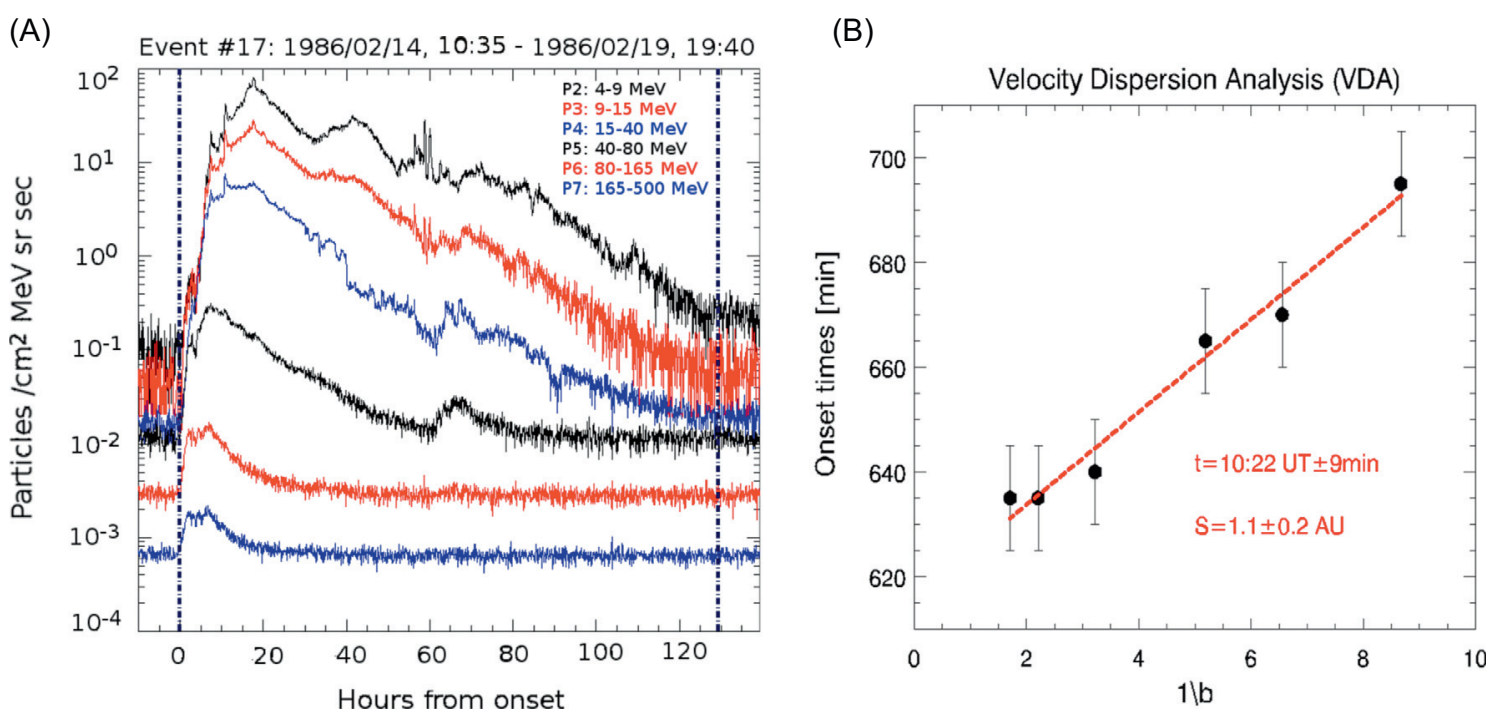

Fig. 2. An illustration of the velocity dispersion analysis (VDA) method applied onto GOES particle measurements. (A) The event no. 17 from Table 2 (supplementary online material) as this was recorded onboard GOES/EPS at a wide energy range from 4-500 MeV (i.e. P2-P7; see the legend of the figure for details). Vertical lines mark the start and end time of the event as identified in this work; the title provides the number of the event as well the start and end time; the ordinate presents the proton intensities (in particles $/ \mathrm{cm}^{2} \mathrm{sr} \mathrm{sec} \mathrm{MeV)} \mathrm{and} \mathrm{the} \mathrm{abscissa} \mathrm{presents} \mathrm{the}$ time in hours from the onset time, the latter labelled with the first vertical line. (B) The application of the VDA method, i.e. start times of the proton event observed by GOES/EPS, as a function of the inverse velocity $(1 / \mathrm{b})$. The straight line shows a linear regression to the observations, where the slope corresponds to the apparent path length $(S)$, and the intersection with the $X$-axis gives the solar release time $(t)$.

evident afterwards. This numerical approach has been validated using GOES NOAA products. In particular, we applied it to GOES differential data (provided by the NOAA server) and re-produced successfully the corresponding integral data. ${ }^{10}$

\subsection{Associating SEPs to their parent SFs}

The next step was to associate SEP events with their parent SFs. In making such associations we aimed to separate the proton enhancements from different SFs to the best of our ability. The attribution of the SEP events to their solar sources was a critical part of the study and was implemented on three steps:

- The first iteration of the SEP association with their parent solar sources was based on the velocity dispersion analysis (VDA) method. VDA of an SEP event is based on determining the onset times of the event at various energies and presenting these onset times as a function of inverse velocity of the particles at respective energies (Vainio et al. 2013). The velocity dispersion equation at $1 \mathrm{AU}$ can be written as:

$$
t_{\text {onset }}(E)=t_{0}+8.33 \frac{\min }{\mathrm{AU}} \times S \times \beta^{-1}(E),
$$

- where $t_{\text {onset }}(E)$ is the observed onset time in minutes at proton kinetic energy $E, t_{0}$ is the release time (min) from the acceleration site, $S$ is the apparent path length (AU) travelled by the particles and $\beta^{-1}(E)=\frac{c}{v}(E)$ is the inverse velocity of the particles. Thus, by linear fitting of the onset times as a function of the corresponding inverse velocity, estimates for both the solar release time (SRT) and the apparent path length of the particles can be obtained. The validity of the VDA method has been an item of active research by several authors (Lintunen \& Vainio

\footnotetext{
${ }^{10} \mathrm{http}: / /$ www.stce.be/esww 11/contributions/public/Session5/S5-P4-SandbergI/poster_ESWW_GOES.pdf
}

2004; Sáiz et al. 2005; Rouillard et al. 2012; Vainio et al. 2013), with the VDA method typically used to estimate the SRT of SEP events (Lario et al. 2014, 2016). For the first time, in our analysis, we have incorporated in the VDA the new and improved $E_{\text {effective }}$ for each of the P2-P7 GOES energy channels that have been obtained by the cross-calibration of GOES/SEM with IMP-8/GME data (Sandberg et al. 2014, their Tabs. 2 and 3 ).

- An illustration of this procedure is presented in Figure 2. Figure 2A shows the SEP event of 14 February, 1986 (event no. 17 in Tab. 2; Supplementary online material) as this was recorded onboard GOES/EPS over a wide energy range from 4 to $500 \mathrm{MeV}$ (i.e., P2-P7; see the legend of the figure for details). Vertical lines mark the start and end times of the event as this was identified in this work; the title provides the number of the event as well as the start and end times; the ordinate presents the proton intensities (in particles/ $\mathrm{cm}^{2}$ sr sec $\mathrm{MeV}$ ) and the abscissa presents the time in hours from the onset time, the latter labelled with the first vertical line. Figure $2 \mathrm{~B}$ presents the application of the VDA method, i.e. start times of the proton event observed by GOES/EPS, as a function of the inverse velocity. The straight line shows a linear regression to the observations, where the slope corresponds to the apparent path length, and the intersection with the $y$-axis gives the solar release time. At this point, one should note that, given the small number of channels made available from the SEM instrument (i.e. six channels) and that the smallest in magnitude SEP events did not present signatures at high energies, an average of four channels per event were left to be used as a seeder for the VDA.

- As a second step, other published SEP event catalogues were used in parallel (cross-evaluated) in an attempt to identify as objectively as possible the parent solar events (e.g., SF) related to a single SEP event. In particular, 
Table 1. References for SEP events catalogues.

\begin{tabular}{|c|c|c|}
\hline $\begin{array}{c}\text { No of } \\
\text { catalogue }\end{array}$ & Paper/List & Reference \\
\hline 1 & A study of solar energetic particle events of 1997-2006: their composition and associations & Cane et al. (2010) \\
\hline 2 & $\begin{array}{l}\text { A technique for short-term warning of solar energetic particle events based on flare location, } \\
\text { flare size and evidence of particle escape }\end{array}$ & Laurenza et al. (2009) \\
\hline 3 & $\begin{array}{l}\text { The first SEPServer event catalogue at } \approx 68-\mathrm{MeV} \text { solar proton events observed at } 1 \text { AU in } 1996 \text { - } \\
2010\end{array}$ & Vainio et al. (2013) \\
\hline 4 & Dr A. Aran, University of Barcelona, SEP list & $\begin{array}{l}\text { Private communication, } \\
2014\end{array}$ \\
\hline 5 & NOAA Space Weather SEP list & $\begin{array}{l}\text { ftp://ftp.swpc.noaa.gov/ } \\
\text { pub/indices/SPE.txt }\end{array}$ \\
\hline 6 & Statistical analysis of solar proton events & Kurt et al. (2004) \\
\hline 7 & Energetic particle and other space weather events of solar cycle 24 & Gopalswamy (2012) \\
\hline 8 & $\begin{array}{l}\text { Relationship between solar energetic particles and properties of flares and CMEs: statistical } \\
\text { analysis of solar cycle } 23 \text { events }\end{array}$ & Dierckxsens et al. (2015) \\
\hline 9 & $\begin{array}{l}\text { Characteristic times of gradual solar energetic particle and their dependence on associated } \\
\text { coronal mass ejection properties }\end{array}$ & Kahler (2005) \\
\hline
\end{tabular}

Table 1 summarizes the SEP catalogues/lists that have been used in this part of our analysis. In practice, when the association stems from literature, we make a note in Table 2 (supplementary online material).

- Furthermore, as a third step, cross-checks for every SEP event in our catalogue from the inspection of multi-plots of SXRs, CME height-time and GOES particle time profiles were applied, in an attempt to validate our derived results and to identify as accurate as possible the parent solar events of every single SEP event. An illustration of such a multi-plot is provided in Figure 3, for the event no. 169 in Table 2 (14 July, 2000; see supplementary online material). (Fig. 3A) The GOES proton flux at $E>10,>30$ and $>60 \mathrm{MeV}$ (red, blue and orange lines, respectively). The black dashed vertical line corresponds to the anticipated solar release time for this event (shifted by $500 \mathrm{~s}$ to allow the comparison with the EM emissions). (Fig. 3B) The SXR flux observed by GOES, denoting an X5 solar flare at N22W07 (red curve; left axis). The black dashed vertical line corresponds to the start time of the solar flare. The dashed blue line provides the height-time plot of the CME leading edge observed by SOHO/LASCO (blue line; right axis), extrapolated back to the surface of the Sun. (Fig. 3C) Radio flux observed by Wind/WAVES. The dashed black line corresponds to the start time of the identified type III burst.

One should note that, between 1984 and 1997 it was possible to associate 67 CMEs-SFs pairs to SEP events. This corresponds to $\sim 50 \%$ of the SEP events marked within this period (67/140). Apart from the importance of including and presenting this information in our database the CME characteristics of this period could not be included in our statistics (Sect. 3) due to lack of homogenization, compared to the entire time span of the study.

\subsubsection{The SEP part of the database}

The SEP part of the database is presented in Tables 2 and 3 (supplementary online material), re-produced as additional online material to this article. Table 2 column 1 (supplementary online material) provides the number of the event,

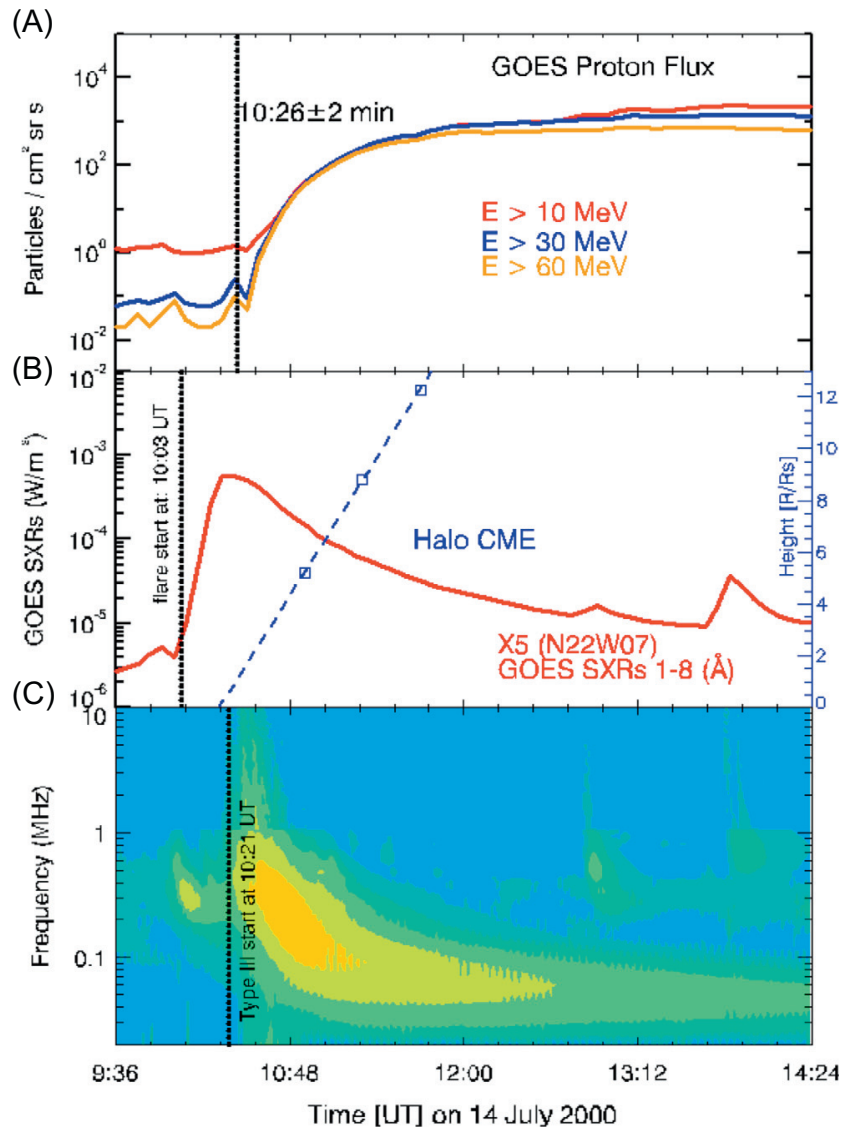

Fig. 3. Event no. 169 of Table 2 (supplementary online material). (A) The GOES proton flux at $E>10,>30$ and $>60 \mathrm{MeV}$ (red, blue and orange lines, respectively). The black dashed vertical line corresponds to the anticipated solar release time for this event (shifted by $500 \mathrm{~s}$ to allow the comparison with the EM emissions). (B) The SXRs flux observed by GOES, denoting an X5 solar flare at N22W07 (red curve; left axis). The black dashed vertical line corresponds to the start time of the solar flare. The dashed blue line provides the height-time plot of the CME leading edge observed by $\mathrm{SOHO} / \mathrm{LASCO}$ (blue line; right axis), extrapolated back to the surface of the Sun. (C) Radio flux observed by Wind/WAVES. The dashed black line corresponds to the start time of the identified type III burst. 
columns 2-4 provide the date of the SEP event occurrence (including the year, date and the start time of the SEP event at 4-8 MeV (P2) differential energy channel), column 5 presents the SEPEM index, resulting from the comparison of the SEPEM reference list to the events included in the SEP part of the database. A zero marks an SEP event that is present in both lists, 1 to $n$ indicates the successive manually broken SEP events resulting from the analysis illustrated in Figure 1; there are two events that although are present in the SEP part of the database are not included in the SEPEM reference list, namely events no. 45 (20 June, 1989) and no. 198 (15 September, 2001). Columns 6-14 display the relevant SF information in terms of flare start time (date and time), rise time, position (longitude, latitude), magnitude (SXR peak flux) and fluence (in $\mathrm{J} / \mathrm{m}^{2}$ ), time of maximum $\left(t_{N}\right)$ and maximum value $\left(I_{N}\right)$ of the derivative of the SXRs (e.g., HXR's proxy; "Neupert effect"). Columns 15 and 16 provide the timing (start and end times) of the type III burst, columns 17 and 18 mark the occurrence (or not) of a type II and/or type IV radio burst, column 19 shows the occurrence (or not) of an SEP event in the electron recordings of Advanced Composition Explorer (ACE)/Electron Proton and Alpha Monitor (EPAM)/Deflected Electrons (DEs), columns 20-22 show the CME information, in terms of a CME index ( 0 in case no. CME was reported and 1 in case a CME was identified), the plane-of-sky CME velocity and the $\mathrm{CME}$ width. Column 23 provides the corresponding reference number to the reference SEP list of Table 1, which is the output of the cross-evaluation of all other available SEP lists. Finally, column 24 provides comments relevant to each of the SEP events (if any). Numbers in this column point to the reported associations which have been used during the identification of the parent solar events and refer also to Table 1. Vertical lines distinguish the information on the SEP event, the associated SF and the CME events. Table 2 (supplementary online material) provides also four indicators concerning data quality: (a) spikes, (b) brief data gap(s), (c) large data gap and (d) missing recordings of a channel. Table 4 presents the calculated proton peak flux (in pfu) and fluence (in $\mathrm{cm}^{-2}$ $\mathrm{sr}^{-1}$ ) at the respective integral energies of $E>10 ; 30 ; 60$ and $100 \mathrm{MeV}$ for each of the $314 \mathrm{SEP}$ events in our catalogue.

\section{Statistical analysis}

\subsection{Dependence of the occurrence of SEP events on their parent} solar sources

\subsubsection{Solar flares - SFs}

The dependence of the expected probability for the SEP occurrence on the heliolongitude of the parent SF has been extensively reported and documented (Belov et al. 2005; Laurenza et al. 2009; Cane et al. 2010; Dierckxsens et al. 2015; Trottet et al. 2015). Figure 4 presents a scatter plot of the SXR peak flux (in $\mathrm{W} / \mathrm{m}^{2}$ ) as a function of the heliolongitude of the identified sources (in degrees). The figure includes all $20429 \geq$ C1.0 flares in the database (see details in Sects. 2.4 and 2.1) covering roughly more than three solar cycles from 1984 to 2013. Red open circles present all flares, whereas black filled circles stand for those SFs associated with the 314 identified SEP events. One may notice the relative lack of SEP-associated SFs in the case of eastern and relatively weak flares (e.g., lower left corner of Fig. 4) whereas the majority of strong western flares are SEP related (e.g., top right corner of Fig. 4). In particular, 42\% (39/93) of all X1.0 SFs west from

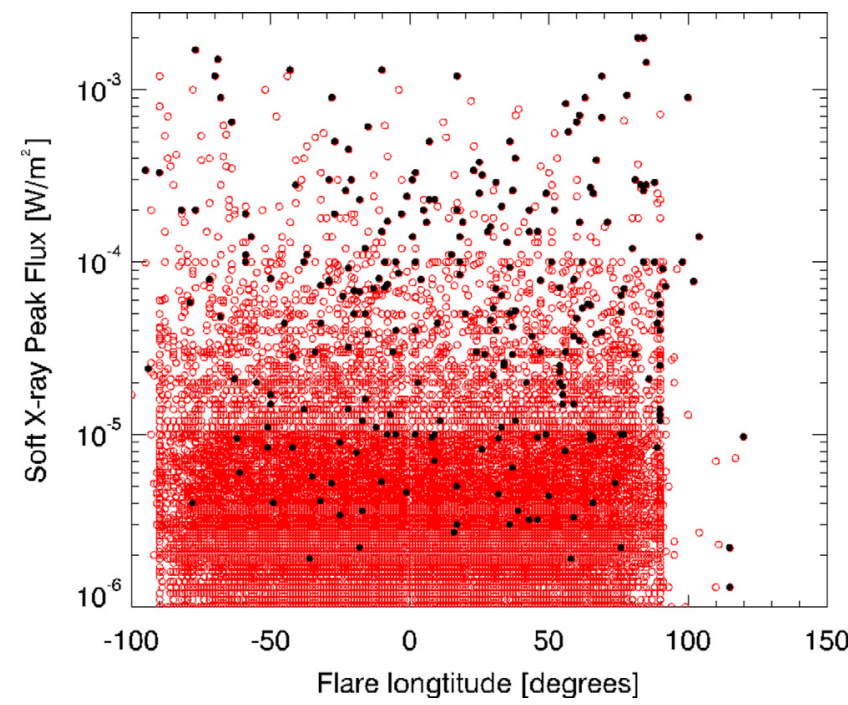

Fig. 4. Distribution of SXR flux (in $\mathrm{W} / \mathrm{m}^{2}$ ) as a function of the flare longitude (in degrees) for all $20429>\mathrm{C} 1.0$ flares in our database from 1984-2013 (red open circles) and for the 314 SEP events (black filled circles).

$30^{\circ}$ result in an SEP event, whereas this percentage falls down to $\approx 0.2 \%(11 / 6234)$ for all $\mathrm{C}$-class $(<\mathrm{M} 1.0)$ SFs east from $-20^{\circ}$. A closer look at Figure 4 reveals more details: the region under the threshold of M-class flares (i.e. $<10^{-5}$ in $\mathrm{W} / \mathrm{m}^{2}$, for the flare flux) is populated with a vast number of non-SEPrelated SFs and with a small number of SEP-related SFs. That means that a small percentage of SEP-related flares is expected to appear in association with C-class SFs. In addition, the percentage of SEP-related flares when the flare flux is $>10^{-5}$ in $\mathrm{W} / \mathrm{m}^{2}$ and corresponds to $\geq \mathrm{M}$-class flares, extends from the centre of the solar disk both to the east and west with a clear preference to the west, where the percentage of SEP-related flares is significantly higher. This is especially prevalent for SFs characterized by a magnitude of $\geq \mathrm{M} 2.0$ west from $45^{\circ}$. The percentage of SEP-related flares is gradually reduced when moving from the centre of the solar disk to the east. It should also be noted that in this case mainly particular strong flares (on average with X1.0 class) are characterized as SEP related. Finally, the majority of SFs with a class of $\geq X 7.0$ are SEP-related even for longitudes west of $-70^{\circ}$.

Figure 5 shows the distribution of the SFs as a function of their longitude (in degrees), in $10^{\circ}$ bins with error bars given by the square root of the number of entries in each bin, for all $20429>\mathrm{C} 1.0$ flares in our sample (Fig. 5A) and for the 314 SEP events (Fig. 5B) and as a function of their position (in both latitude and longitude) of the non-SEP-related SFs for the whole SEP part of the database (open red circles - Fig. 5C) and the SEP-related ones (black filled circles - Fig. 5D). First of all, one can see that there is a zone between -40 and $40^{\circ}$ in latitude that holds all SFs, together with the SEP-related ones - which is typical for source regions (Belov et al. 2005). Since $\mathrm{SFs}$ are mainly coming from active regions, Figure $5 \mathrm{C}$ essentially outlines the active-region belt of the Sun (Maunder 1904). Furthermore, it is clearly visible that a number of SEP events are associated with SFs located very close to the limb of the solar disk $\left(\geq 70^{\circ}\right.$ and $\left.\leq-70^{\circ}\right)$ and on the nonvisible part of it, i.e., back side of the Sun. This means that even at such remote locations SFs can contribute to SEP events that are registered at Earth. In contrast to the RelEASE system (Posner 2007), this is also a factor of large uncertainty for forecasting 
(A)

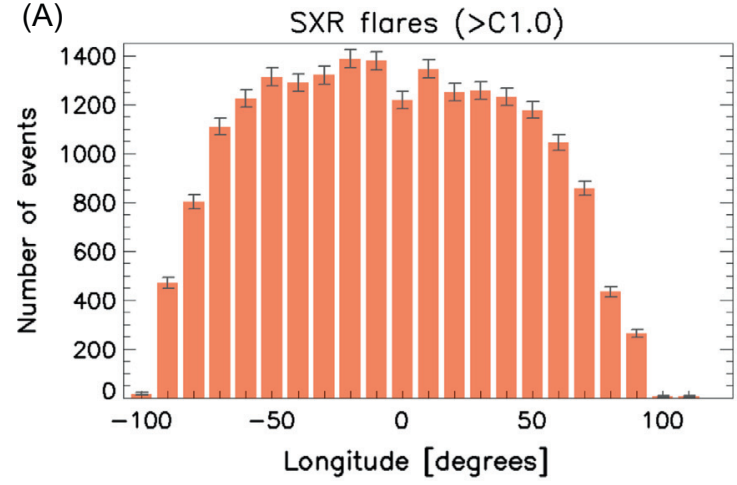

(C)

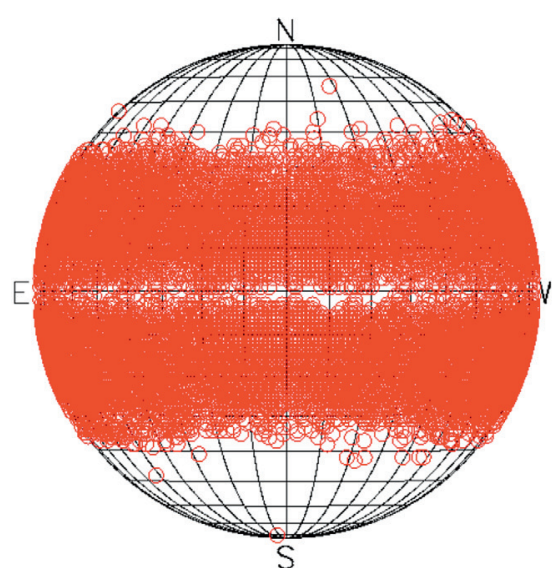

(B)

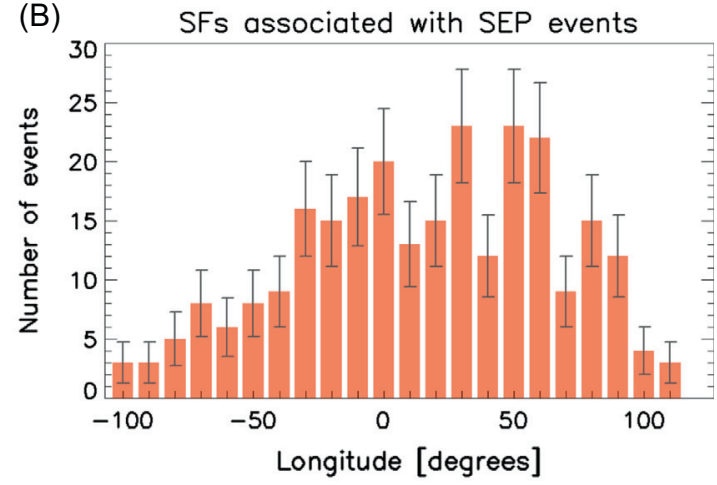

(D)

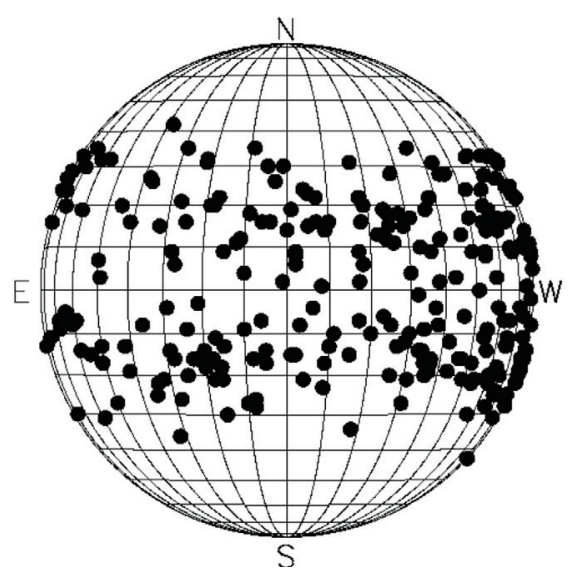

Fig. 5. Distribution of the solar flare heliolongitudes (in $10^{\circ}$ bins) for all $20429>$ C1.0 flares in our sample from 1984-2013 (A) and for the 314 SEP events (B). The error bars denote the statistical error. Solar flare latitude (in degrees) as a function of the flare longitude (in degrees) for all $20429>$ C1.0 flares in our database from 1984-2013 (red open circles) (C) and for the 314 SEP events (black filled circles) (D).

systems of SEP events that rely on near real-time data of SFs as they are either unable to identify an event (e.g., SF) at the back side of the Sun or underestimate its magnitude once its location exceeds the limit of $70^{\circ}$. Finally, when comparing between Figures $5 \mathrm{~A}$ and $5 \mathrm{~B}$, a lower number of SEP events arising from SFs located in the eastern hemisphere, with respect to those in the western hemisphere, are observed. There is a large spread of SEP events from the $\mathrm{E} 30^{\circ}-\mathrm{W} 30^{\circ}$ region. As expected (Belov et al. 2005), the western hemisphere includes a second peak at $\mathrm{W} 50^{\circ}-\mathrm{W} 60^{\circ}$, where the SEP occurrence is prevalent, which is not seen in the eastern hemisphere. The disparity is maintained until the limb. As a matter of fact, the footpoints of the interplanetary magnetic field (IMF) lines, connecting the Sun to the Earth, fall into the longitude interval $\mathrm{W} 45^{\circ}-\mathrm{W} 65^{\circ}$ (Parker 1965) for nominal solar-wind (SW) conditions (e.g., $400 \mathrm{Km} / \mathrm{s}$ ), whereas for different SW speeds (250-800 $\left.\mathrm{km} \mathrm{s}^{-1}\right)$, they span a wider longitudinal range $\left(\mathrm{W} 25^{\circ}-\mathrm{W} 85^{\circ}\right)$. As a result, SEP events have a significant probability of being recorded near Earth following SFs located in the western hemisphere of the Sun. Figure 5B demonstrates that SEP events associated with the western SFs are more frequent by a factor of $\approx 4$ than the eastern ones in the case of the W70-W90 bin and the E70-E90 one. Indeed, when comparing the number of events within each bin the relevant numbers are 48 over 13 which lead to a factor of 3.96. These results are in line with results reported, for instance, in Belov et al. (2005), Laurenza et al. (2009), Park et al. (2010) and Dierckxsens et al. (2015), among others.

\subsubsection{Coronal mass ejections - CMEs}

Figure 6A presents the distribution of the non-SEP-related CME events and that of the SEP-related CMEs (Fig. 6B) observed in the SOHO era, in the database, as a function of their speed (in $\mathrm{km} \mathrm{s}^{-1}$ ). It can be seen that most of the nonSEP-related CMEs present an average velocity of $500 \mathrm{~km} \mathrm{~s}^{-1}$, whereas the SEP-related CMEs are faster with the majority of the events $\left(111 / 158^{11}\right.$ events $)$ having a velocity $\geq 1000 \mathrm{~km} \mathrm{~s}^{-1}$, the average velocity being $\sim 1390 \mathrm{~km} \mathrm{~s}^{-1}$, see Figure 6B. Moreover, this finding conveys that typical SEP-associated CMEs stem almost exclusively from solar active regions (hence the association with SFs), where CME velocities exceed $750 \mathrm{~km} \mathrm{~s}^{-1}$ (Sheeley et al. 1999) (135/158 events of this work) or $\sim 900 \mathrm{~km} \mathrm{~s}^{-1}$ (Georgoulis 2008) (126/ 158 events). Nonetheless, as it is shown in the left part of Figure 6B, several slow CMEs are also associated with SEP events, although the association is low. Next the CME size, as this is expressed by the width of the CME, is considered. Figure 6 presents the distribution of the non-SEP-related CME events (Fig. 6C) and the corresponding distribution for the SEP-related CME events (Fig. 6D) observed in the SOHO era, in the database. Evidently, most SEP events are associated with halo CMEs (i.e., a width of $\left.360^{\circ}\right)(106 / 158$ events) (Park et al. 2012). As a result, both fast and halo CMEs form a favourable condition for the acceleration of particles that will result in SEP events since this is a prerequisite for the

\footnotetext{
${ }^{11} 158$ represents the total number of SEP events in our catalogue for which complete CME information was available.
} 
A. Papaioannou et al.: SEP events and their parent solar sources

(A)

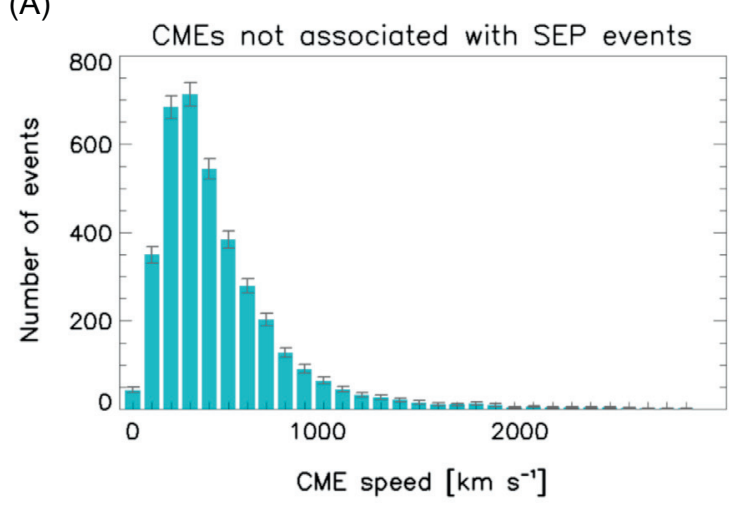

(C)

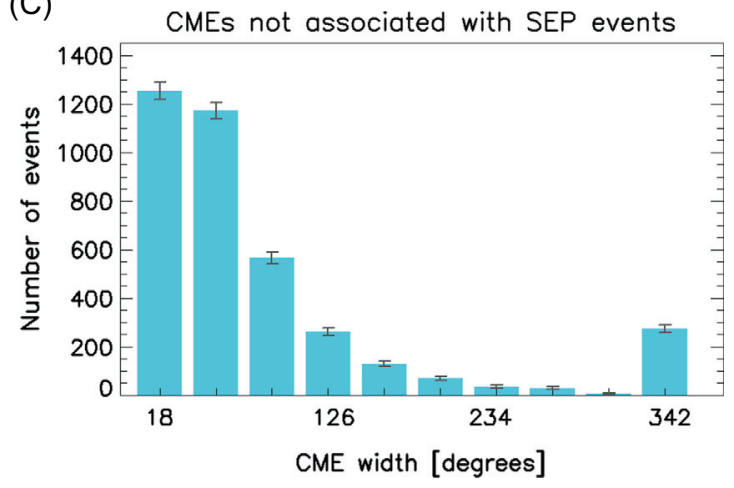

(B)

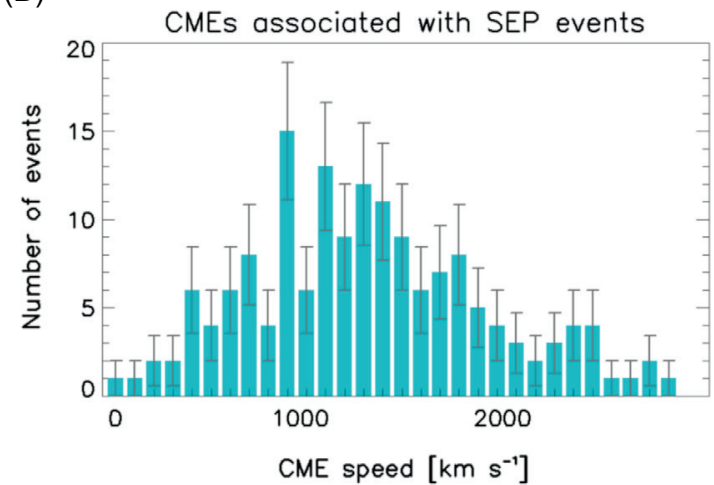

(D)

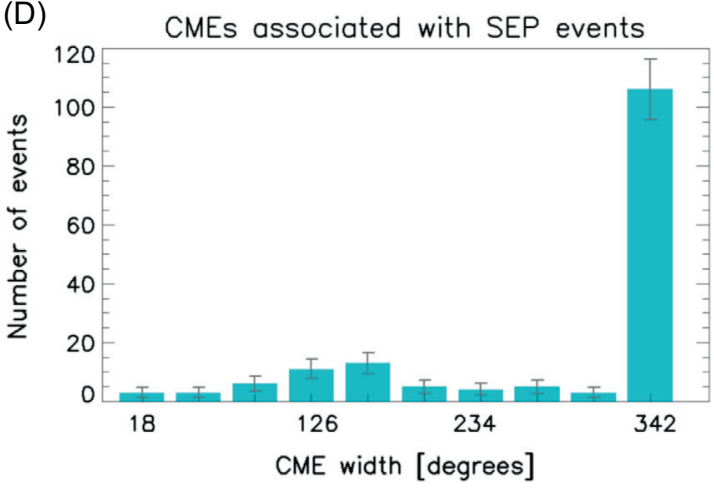

Fig. 6. Distribution of the CME velocity (in $\mathrm{km} \mathrm{s}^{-1}$ ) for all 3535 CMEs not associated with SEP events in our sample from 1997-2013 (A) and for the 158 SEP events, within the same time period (B). Distribution of the CME width (in degrees) for all 3535 CMEs not associated with SEP events in our sample from 1997-2013 (C) and for the 158 SEP events, within the same time period (D). The error bars denote the statistical error.

establishment of a shock, driven by the corresponding CME event (Kahler 2001; Park et al. 2012). At this point it is important to note that all CME observations are the projection of the three-dimensional erupting structure on the plane of the sky. Therefore, the measured (width, height and brightness) and derived (speed, mass and energy) quantities are also projected on the plane and represent lower limits of the true, unprojected CME properties (Colaninno \& Vourlidas 2009).

\subsection{Dependence of the proton peak flux}

\subsubsection{SF characteristics}

Figure 7 illustrates the proton peak flux at various integral energies, i.e., $E>10$ (presented as red triangles), 30 (presented as blue triangles), 60 (presented as orange triangles) and 100 (presented as magenta triangles) $\mathrm{MeV}$ as a function of: the SXR peak flux (in $\mathrm{W} / \mathrm{m}^{2}$ ) (Fig. 7A); the duration of the associated SF (in minutes) (Fig. 7B); the rise time of the SF (Fig. 7C) and the longitude (in degrees) of the SF (Fig. 7D). The NOAA classification of solar radiation storms, ${ }^{12}$ which is based on the $E>10 \mathrm{MeV}$ proton flux level, has also been considered, with the corresponding storm levels (e.g., S1-S4) indicated in Figure 7A. The rise time of the solar flare, that is the time that results from the subtraction of the SF peak time minus its onset time, is a measure of its impulsiveness.

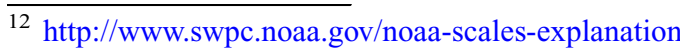

In particular, Park et al. (2010) defined the time threshold for the characterization of the impulsive time of SFs to $<0.3 \mathrm{~h}=18 \mathrm{~min}$.

Our results can be summarized as follows:

- A wide range of proton peak fluxes are present in the database from 0.1 to $2 \times 10^{4}$ pfu. Stronger flares (with class $\geq$ M1.0) tend to lead to higher peak fluxes at all energies with the strongest of all flares (with class $\geq X 1.0$ ) marking the highest peak fluxes at each integral energy channel. This means that there is a gradual rise in the expected proton peak flux of each integral energy channel with respect to the class of the parent solar flare. In other words, stronger flares result in more significant solar radiation threats. This is illustrated in Figure 8 which depicts the mean values $(P \pm \delta P)$ of the proton peak fluxes (in pfu) at several integral energies $(E>10 ;>30 ;>60$ and $>100 \mathrm{MeV}$ ) and their respective standard deviation of the mean, as a function of the mean SXR peak flux (in $\mathrm{W} / \mathrm{m}^{2}$ ) for the solar flares associated with SEP events in the SEP part of the database.

- Figure 9 shows the proton peak flux (in pfu) of the SEP events in the SEP part of the database for $E>10 \mathrm{MeV}$ (Fig. 9A) and $E>100 \mathrm{MeV}$ (Fig. 9B), as a function of the rise time of the solar flare (in minutes). The vertical dashed black line, in both panels, indicates a rise time of $18 \mathrm{~min}$ - the limit suggested by Park et al. (2010). It is important to note that although the NOAA definition that results in the definition of S1-S5 levels is based on the $E>10 \mathrm{MeV}$ proton peak flux surpassing the limit of 
(A)

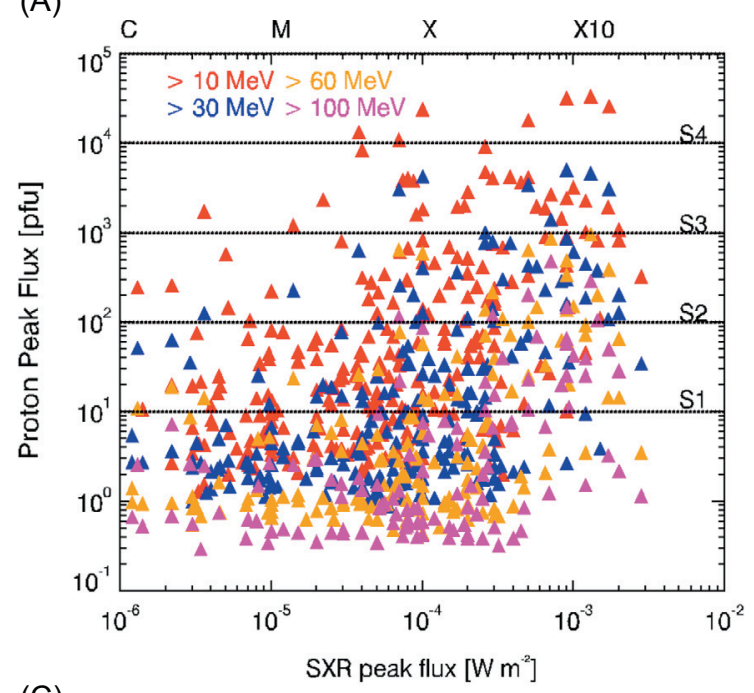

(C)

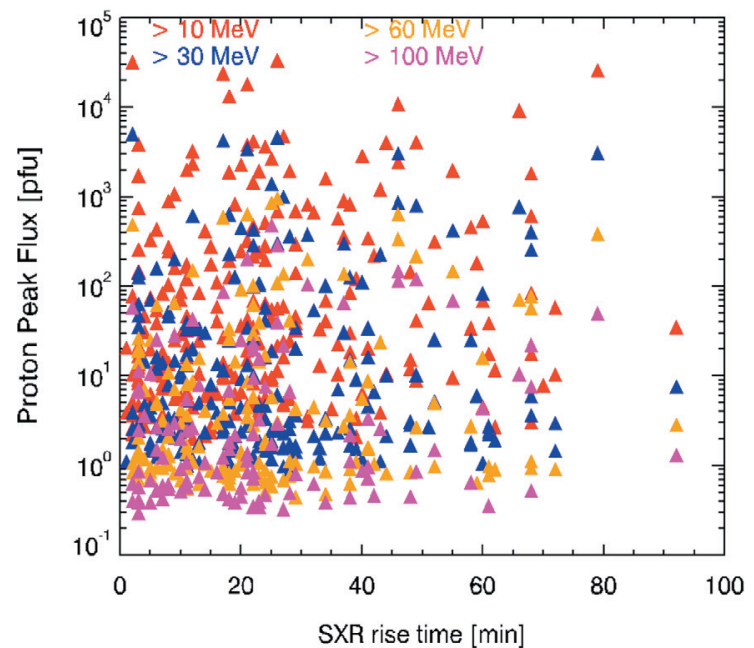

(B)

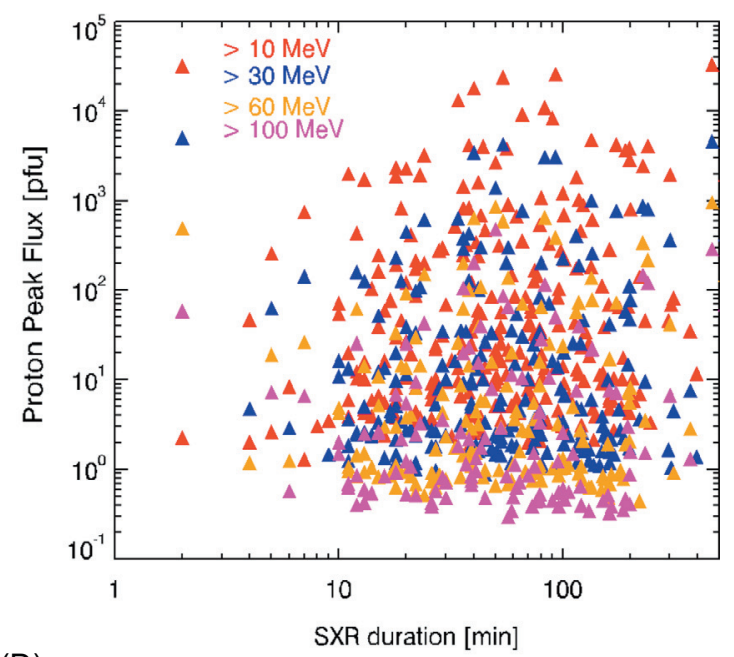

(D)

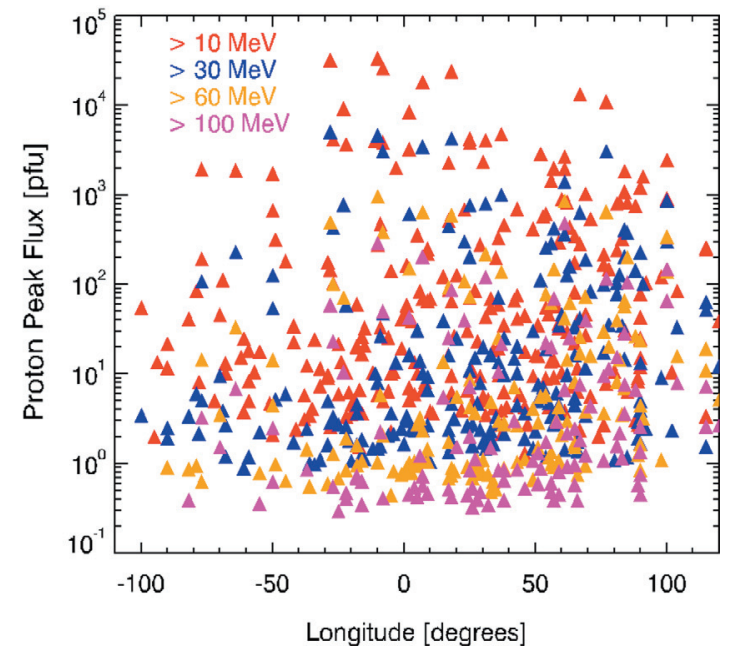

Fig. 7. The proton peak fluxes of the SEP events in the SEP part of the database for various integral energies $(>10 ; 30 ; 60$ and $100 \mathrm{MeV})$ as a function of the SXR peak flux (in $\mathrm{W} / \mathrm{m}^{2}$ ) (A); the duration of the associated parent solar flares (in minutes) (B); the rise time of the associated parent solar flares (in minutes) (C) and the longitude of the parent solar flare (in degrees) (D). (A) Incorporates the NOAA Space Weather Scales for Solar Radiation Storms (S1-S4), as horizontal dotted lines labelled with each scale, i.e. S1-S4; see http://www.swpc.noaa.gov/noaascales-explanation for details. Furthermore, it incorporates the class, i.e. C, M, X and X10, of solar flares on its top part.

$10 \mathrm{pfu}$, we scaled this limit for the $E>100 \mathrm{MeV}$, by one order of magnitude in accordance with the alert issued by the NOAA Space Weather SEP Alert system, ${ }^{13}$ that is triggered, for $E>100 \mathrm{MeV}$ SEP events that surpass the limit of 1 pfu. The horizontal black line at each of the two panels highlights a threshold of 10 and $1 \mathrm{pfu}$ for $E>10 \mathrm{MeV}$ and $E>100 \mathrm{MeV}$, respectively. At an integral energy of $E>10 \mathrm{MeV}$, there are $127 \mathrm{SEP}$ events in the database associated with impulsive solar flares, as well as, 152 SEP events associated with gradual solar flares, based on the classification introduced by Park et al. (2010) (Fig. 9A). Thirty-five SEP events are lacking an associated SXR rise time (see Tab. 2, online supplementary material). Fifty SEP events associated with impulsive SFs, i.e. 50/127 ( $\approx 39.3 \%$ ) (orange triangles pointing down) and 38 SEP events associated with gradual SFs, i.e. $38 / 152(\approx 25 \%)$ (purple diamonds), result in relatively low proton peak fluxes $(\leq 10 \mathrm{pfu}$ for $>10 \mathrm{MeV}$ proton peak flux). On the other hand, $77 / 127(\approx 60.7 \%)$ SEP

\footnotetext{
${ }^{13} \mathrm{http} / / /$ legacy-www.swpc.noaa.gov/alerts/AlertsTable.html
}

events associated with impulsive SFs (red circles) and $114 / 152(\approx 75 \%)$ associated with gradual SFs (blue triangles pointing up) result in proton peak fluxes that surpass the limit of $>10 \mathrm{pfu}$. The situation is similar for an integral energy of $E>100 \mathrm{MeV}$ (Fig. 9B).

- Figures 9C and 9D shows the percentage $P_{i}$ of $E>10 \mathrm{MeV}(E>100 \mathrm{MeV})$ SEP events associated with gradual and impulsive SFs, computed for all four radiation levels $S_{i}(i=1$ to 4$)$ as follows: $P_{i}=N_{i \mathrm{SEP}} / N_{i}$, where $N_{i}$ is the total number of solar eruptive events in the subset $i$ and $N_{i_{\text {SEP }}}$ is the number of those events that resulted in an observed SEP event. The respective error is $\pm \sqrt{P_{i}\left(1-P_{i}\right) / N_{i}}$, corresponding to a $68 \%$ confidence level when the sample is described by a normal distribution (Dierckxsens et al. 2015). Our analysis shows that the percentage of SEP events associated with gradual SFs at all four radiation levels transcend the relevant percentage of the SEP events associated with impulsive SFs by an average factor of 1.66 for $E>10 \mathrm{MeV}$ and $1.38 E>100 \mathrm{MeV}$, respectively. It should be noted, 


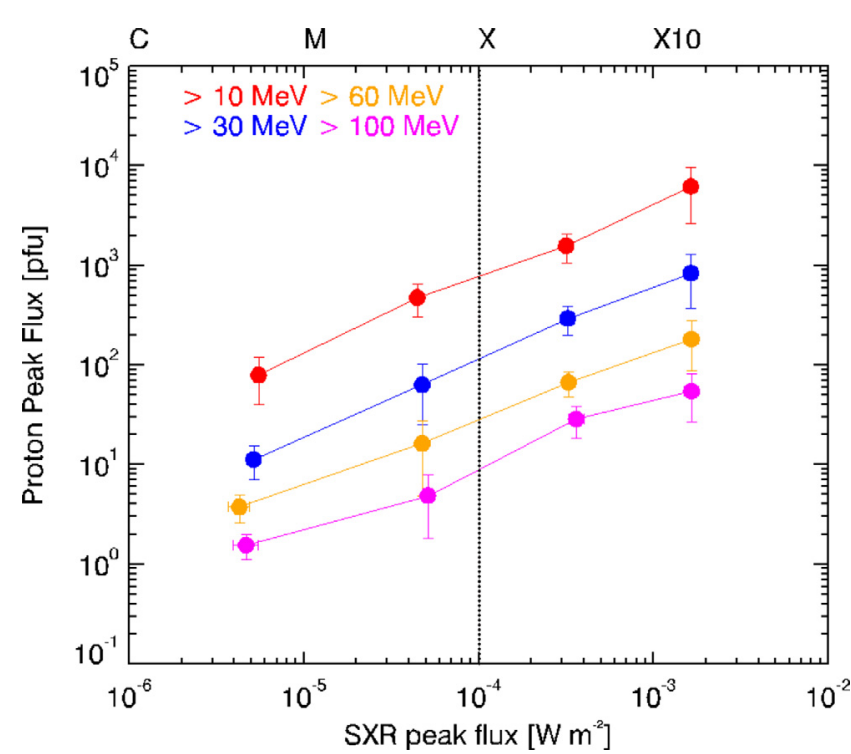

Fig. 8. Mean values of the proton peak fluxes (in pfu) at several integral energies $(E>10 ;>30 ;>60$ and $>100 \mathrm{MeV})$ and their respective standard deviation of the mean, as a function of the mean SXR peak flux (in $\mathrm{W} / \mathrm{m}^{2}$ ) for the solar flares associated with SEP events in the SEP part of the database. Each integral energy is color coded as follows: $E>10 \mathrm{MeV}$ with red, $E>30 \mathrm{MeV}$ with blue, $E>60 \mathrm{MeV}$ with orange and $E>100 \mathrm{MeV}$ with magenta color. The class of the solar flares is indicated at the top part of the figure, labelled as $\mathrm{C}, \mathrm{M}, \mathrm{X}$ and $\mathrm{X} 10$. The dashed vertical black line indicates the lower limit of the X-class flares.

though that impulsive SFs seem to be dominant for $E>100 \mathrm{MeV}$ for $\mathrm{S} 1$ and $\mathrm{S} 2$. However, there is a clear transition of this situation in the S3 level that leads to an average outrun of gradual SFs. As a result, SFs that exhibit a long rise time $(>18 \mathrm{~min})$ are slightly more associated with higher proton peak fluxes, to a greater extent. However, both categories have a strong presence at all radiation levels.

- Figure 10 presents the mean values of the proton peak fluxes (in pfu) at several integral energies and their respective standard deviation of the mean, as a function of the mean SXR duration (in minutes), similar to Figure 8. We have selected four bins for the SXR duration $\left(t_{\text {dur }}\right)$, that is: $t_{\text {dur }}<30, \quad 30 \leq t_{\text {dur }}<60,60 \leq t_{\text {dur }}<90$ and $t_{\text {dur }} \geq 90 \mathrm{~min}$ (Belov et al. 2005). The dependence (or not) of the proton peak flux on the SXR duration of the associated SF seems to be inconclusive. This result highlights the fact that the duration of SFs is a particularly uncertain parameter, as concerns SEP events, since it almost certainly - includes non-flare contribution with the cooling time of the flaring loops affecting the duration of the SF event (Benz 2008).

- Figure 11 presents histograms of the proton peak fluxes (in pfu) at several integral energies $(E>10 ;>30 ;>60$ and $>100 \mathrm{MeV})$, as a function of the longitude of the associated SF, in $10^{\circ}$ bins from -100 to $120^{\circ}$. The proton peak flux of an SEP event is not controlled by the longitude of the associated SF. Although, a preferable location of a SF on the western hemisphere of the solar disk enhances the probability of occurrence for an SEP event, it seems that the longitude of the SF is less critical in determining the proton peak flux of the SEP event.

\subsubsection{CME characteristics}

Figure 12 presents the logarithm of the SEP proton peak fluxes in different integral energies with respect to the speed of the associated CME. The correlation of proton peak fluxes with CME speed has been established in several studies of gradual SEP events (Reames 1999; Kahler 2001). Each panel of Figure 12 provides the calculated Pearson's correlation coefficient $(c c)$, the standard error $\left(S E_{c c}\right)$ and the obtained linear fit. $S E_{c c}=\sqrt{\left(1-c c^{2}\right) /(n-2)}$ with $n$ being the number of pairs used for the calculation of $c c$. From Figure 12 one may notice that for a given CME speed the range of the associated logarithm of the proton peak fluxes at all energies varies significantly especially when moving from lower to higher energies, suggesting that factors other than the CME speed are important for the proton peak fluxes of SEP events. Several of these factors have been discussed in detail by Kahler (2001). In Figure 13A the calculated proton peak flux as a function of the CME width (in degrees) for several integral energies $(>10 ; 30 ; 60$ and $100 \mathrm{MeV})$ is shown. As it has already been noted, most SEP events are associated with halo CMEs. Figure 13A shows that the majority of the large proton peak fluxes (e.g., $>500 \mathrm{pfu}$ ) at any integral energy level are attributed almost solely to such (halo) CMEs. Moreover, Figure 13B presents the mean values of the proton peak fluxes (in pfu) at several integral energies and their respective standard deviation of the mean, as a function of the CME width (in degrees), similar to Figure 8 . We have applied three bins on the CME width, $(w)$, i.e. $w<120^{\circ}, 120^{\circ} \leq w \leq 359^{\circ}$ and $w=360^{\circ}$. It seems that the highest mean proton peak flux at any integral energy is associated to a CME with $w=360^{\circ}$, with the trend decreasing when moving from lower $(E>10 \mathrm{MeV})$ to higher $(E>100 \mathrm{MeV})$ energies. However, the spreading is significant as denoted by the respective errors. In particular, comparing the mean proton peak flux between the two outlier bins (i.e. mean values at $w=360^{\circ}$ divided by the mean values at $w<120^{\circ}$ ) at each integral energy we derived a factor and a propagating error of: $3.34 \pm 2.73(E>10 \mathrm{MeV}), 2.19 \pm 1.45$ $(E>30 \mathrm{MeV}), 2.78 \pm 1.85(E>60 \mathrm{MeV})$ and $1.24 \pm 0.86$ $(E>100 \mathrm{MeV})$.

\subsection{Dependence of the SEP fluence}

The proton fluence that is measured at a near-Earth spacecraft is the outcome of the integration of the integral energy time profile over the time interval of the SEP event, i.e., complete duration of the event from its starting point (onset time) to its end (end of the proton increase) (Smart et al. 2006). In the course of the implementation of the SEP catalogue presented in this work, every effort has been made in order to clearly distinguish between multiple SEP events. However, in such cases it is possible that a further enhancement is present only at low energies, right after a considerably large SEP event (e.g., Fig. 1B). Nonetheless, in all cases, the integration was performed from the onset time of the considered SEP event to the point before the successive identified SEP event (for the case illustrated in Fig. 1B the fluence for the SEP event no. 237 was computed over the time period delimited by the dashed lines).

\subsubsection{SF characteristics}

Figure 14 presents the calculated fluence for each of the events in the SEP part of the database at different energy 
(A)

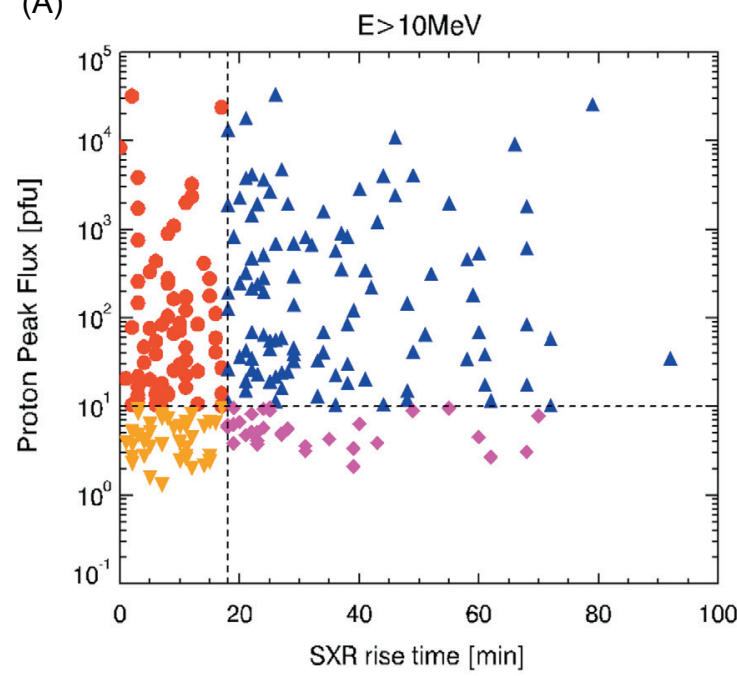

(C)

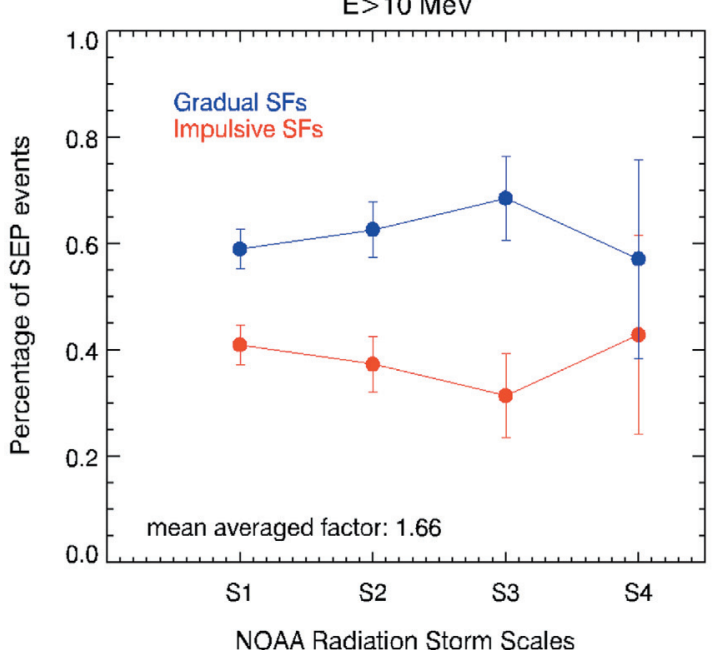

(B)

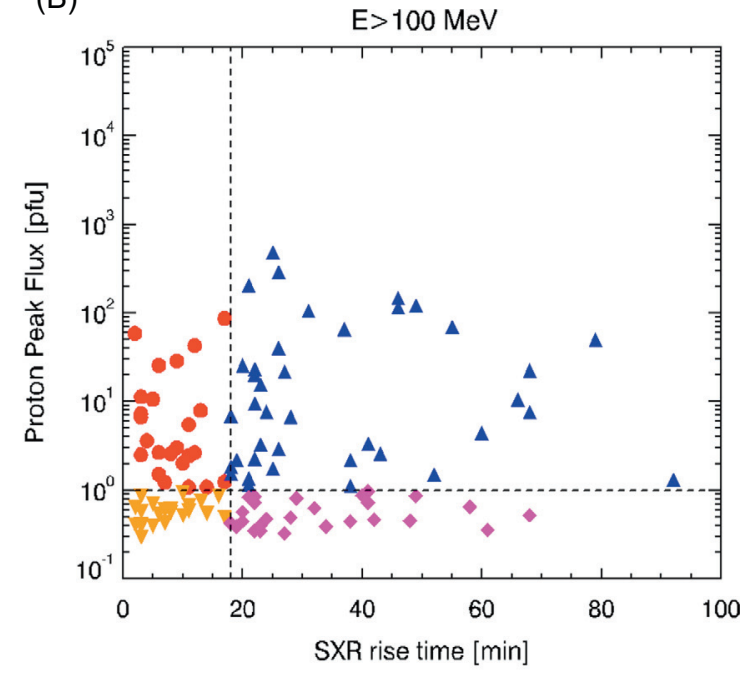

(D)

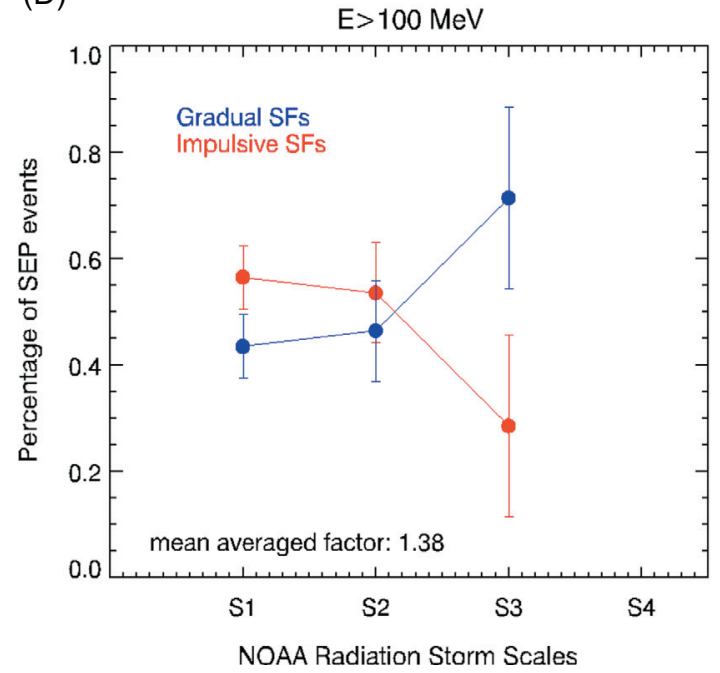

Fig. 9. The proton peak flux (in pfu) of the SEP events in the SEP part of the database for $E>10 \mathrm{MeV}$ (A) and $E>100 \mathrm{MeV}$ (B), as a function of the SXR rise time (in minutes). The vertical dashed black line, in both (A) and (B), indicates a rise time of 18 min. The horizontal black line highlights a threshold of 10 and 1 pfu for $E>10 \mathrm{MeV}$ and $E>100 \mathrm{MeV}$, respectively. (C), (D) SEP occurrence probabilities and their respective errors as a function of the NOAA radiation storm scales. Different symbols are used for each quadrant in (A) and (B), (see text for details).

thresholds: $E>10$ (red triangles), 30 (blue triangles), 60 (orange triangles) and 100 (magenta triangles) $\mathrm{MeV}$ with respect to the X-ray flux, which in turn is a measure of the strength of the parent SFs (Fig. 14A); the rise time, i.e. the impulsiveness of the associated SF (Fig. 14B); the duration of the associated SF (in minutes) (Fig. 14C) and the location (longitude) of the parent SF (Fig. 14D).

Our results indicate that:

- The majority of the events that extend over a threshold of significant fluence (e.g., $>10^{6} \mathrm{~cm}^{-2} \mathrm{sr}^{-1}$ ) are associated with SFs with a rise time larger than the impulsive time (i.e. $>18 \mathrm{~min}$ ). For example, there are 176 SEP events at $E>10 \mathrm{MeV}$ that surpass this threshold. SEP events (158/176) also have a valid rise time in the database, out of which $97(97 / 158,61 \%)$ are associated with gradual solar flares. When an SEP event is observed, more enhanced levels of radiation are expected for gradually rising SFs, than for impulsive ones.
- The highest fluence at all energies is achieved for longlasting flares (SF rise time $>18 \mathrm{~min}$ ) (Park et al. 2010). This implies that, long-lasting SFs that are associated with strong shocks which, in turn, are usually associated with CMEs (Cane et al. 2010), lead more often to enhanced fluence levels.

- Significant fluence is achieved for relatively strong flares ( $\geq \mathrm{M} 1.0$ ), which suggests that the higher the flare flux the higher the resulted fluence of the SEP event, especially in very high proton energy integral thresholds.

Figure 15A depicts the fluence of all SEP events in our database at $E>30 \mathrm{MeV}$ as a function of time, from 1984 to 2013. It appears that the majority of the events in the $E>30 \mathrm{MeV}$ energy range have fluences within $10^{5}-10^{6} \mathrm{~cm}^{-2} \mathrm{sr}^{-1}(146 / 227,64 \%)$ (denoted by the dashed horizontal black lines in Fig. 15A). The most powerful and large SEP events, with energies $>30 \mathrm{MeV}$, that constitute a direct space weather risk, have a fluence that is at least 2 orders 


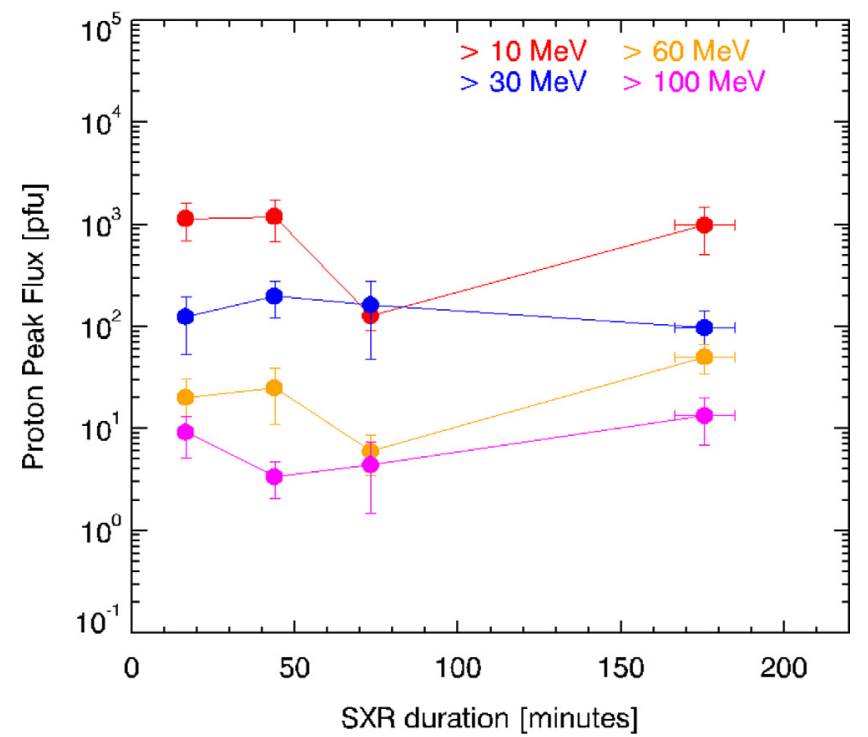

Fig. 10. Mean values of the proton peak fluxes (in pfu) at several integral energies $(E>10 ;>30 ;>60$ and $>100 \mathrm{MeV})$ and their respective standard deviation of the mean, as a function of the mean SXR duration (in minutes) for the solar flares associated with SEP events in the SEP part of the database. Each integral energy is color coded as in Figure 8.

of magnitude larger than the fluence achieved by the majority of the events, i.e., $>10^{8} \mathrm{~cm}^{-2} \mathrm{sr}^{-1}$ (presented with a solid black line in Fig. 15A) (Smart et al. 2006). The evolution of the solar cycle (SC) is also presented in Figure 15A, with the black filled circles that stand for the monthly averaged sunspots and their corresponding smoothed curve (in red). Each $\mathrm{SC}$ is also marked in the figure. There are only a handful of events that exceed this high threshold in the SEP part of our database and thus such events are very rare. These very large events only occur a few times in a SC (i.e. SC22 and SC23), or (in the case of SC21 and SC24) may not occur, at all, in a SC. Quite remarkably, most of these events $(4 / 5=80 \%)$ have an associated solar source that arises from the central part of the solar disk, while the parent solar event of only one event $(1 / 5=20 \%)$ is located in the western hemisphere. Figure $15 \mathrm{~B}$ presents a distribution of the events exceeding the limit of $>10^{8} \mathrm{~cm}^{-2} \mathrm{sr}^{-1}$ for $>30 \mathrm{MeV}$ (in blue) and $>10 \mathrm{MeV}$ (in red) and indicates that severe radiation risk stems from SEP events originating from the central part of the visible solar disk. This result is consistent with the picture introduced by Smart et al. (2006), according to which there should be two basic categories of events: the near-Sun injection source events and the interplanetary shock dominated ones. The former refer to the well-connected SEP events, originating in the western part of the visible solar disk. Particles in these events arrive rather promptly - given the good connection - and the SEP event is brief due to the westward motion of the associated CME. As a result the influence time of the intense particle flux and the corresponding accumulated fluence is also brief. The latter category of events requires the presence of a fast interplanetary shock that continuously feeds the magnetic field lines that connect the shock to the observer with accelerated particles. This lasts for the whole time that is required for the shock to reach and passes beyond the observer point (e.g., near-Earth spacecraft). As a result, given the poor connection, the particle flux at the spacecraft is initially small but it picks up as the shock approaches the observing point. Such events are long lasting in terms of duration. In particular, in our database the only event in the first category is no. 177 (08 November, 2000), which was associated with an M7.0 solar flare at N10W77. In the second category, there are four events: no. 59 (19 October, 1989; associated with an X13 SF at S27E10), no. 169 (14 July, 2000; associated with an X5.0 SF at N22W07 and a Halo CME with a speed of $1674 \mathrm{~km} \mathrm{~s}^{-1}$ ), no. 205 (04 November, 2001; associated with an X1.0 SF at N06W18 and a Halo CME with a speed of $1810 \mathrm{Km} \mathrm{s}^{-1}$ ) and no. 238 (28 October, 2003; associated with an X17.2 SF and a Halo CME with a speed of $2459 \mathrm{Km} \mathrm{s}^{-1}$ ).

\subsubsection{CME characteristics}

Our results (not shown) indicate that fast CMEs are associated with enhanced fluence levels. In particular, if a threshold of $>10^{6} \mathrm{~cm}^{-2} \mathrm{sr}^{-1}$ is assumed for the fluence of the SEP events, most of the events - at all energies (e.g., $E>10 \mathrm{MeV} \mathrm{53/71}$ (75\%), $E>30 \mathrm{MeV} 33 / 37$ (89\%), $E>60 \mathrm{MeV} \quad 19 / 19$ $(100 \%)$ and $E>100 \mathrm{MeV} 9 / 9(100 \%))$ - are associated with fast CMEs (e.g., $\geq 1000 \mathrm{Km} \mathrm{s}^{-1}$ ). Therefore, a significant CME velocity would most probably lead to enhanced radiation levels and constitutes a factor of enhanced radiation risk. Furthermore, most of these fast CMEs have an angular width of $360^{\circ}$ (i.e. $E>10 \mathrm{MeV} 42 / 53$ (79\%), $E>30 \mathrm{MeV} 27 / 33$ $(82 \%), E>60 \mathrm{MeV} 14 / 19(74 \%)$ and $E>100 \mathrm{MeV} 7 / 9$ $(78 \%))$. As a result, fast CMEs with an angular width of $360^{\circ}$ constitute a significant radiation threat.

\section{Origin of SEP events}

Element abundances in SEP events constitute a valuable tool for the study of the underlying physical processes of particle acceleration and release (Reames 1995; Cliver 1996; Reames 1999, 2015). In particular, gradual SEP events have an $\mathrm{Fe} / \mathrm{O}$ ratio $\sim 0.1$, similar to the value in the ambient corona and solar wind (Cane et al. 2010), and are well associated with interplanetary shocks. On the other hand, impulsive SEP events are $\mathrm{Fe}$-rich with $\mathrm{Fe} / \mathrm{O}$ ratios greater than those of the corona and solar wind $(\mathrm{Fe} / \mathrm{O} \sim 1)$ and are not associated with shocks. Such enhanced abundances are interpreted in terms of an acceleration process in SFs, with the particles interacting with resonant waves (Reames 1999; Cane et al. 2010). Furthermore, impulsive SEP events should (in theory) lack CMEs and corresponding shock signatures (e.g., type II radio bursts), with the dominant characteristic being the presence of type III radio bursts that signify the escape of electrons into open magnetic field lines (Cliver 1996; Reames 1999; Cane et al. 2010). Finally, given the large-scale shock acceleration, gradual $S E P$ events are expected to be seen from anywhere on the solar disk, in contrast to impulsive SEP events that would originate in a limited longitude range of a few tens of degrees wide (Cane et al. 2010).

Recently, Cane et al. (2010) used the Fe/O ratio (0.3-0.6, 1.3-3.1, 14 and $34 \mathrm{MeV} /$ nucleon) at the event onset, together with, the time profiles of the SEP events and their abundances, as well as, the ratio of electrons $(\approx 0.5 \mathrm{MeV})$ to protons $(\approx 25 \mathrm{MeV})(e / p)$, to divide the SEP events of their sample into five different groups. First, we mapped the events of our catalogue, with respect to their grouping, for the time span 1997-2006 (this is the range of the Cane et al. (2010) catalogue 
(A)

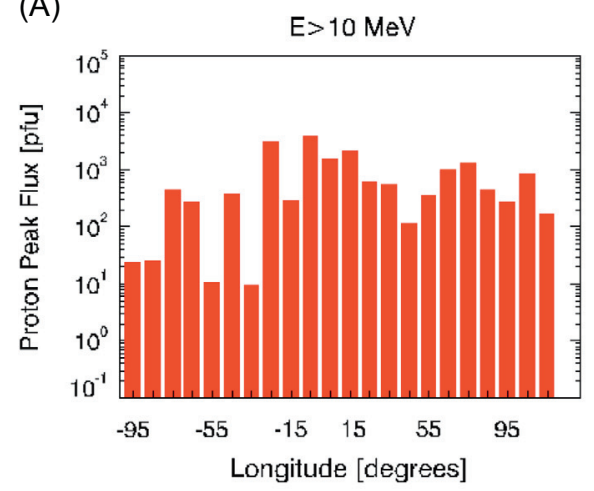

(C)

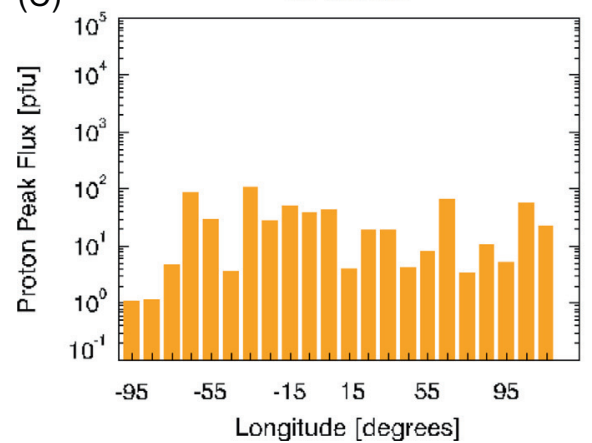

(B)

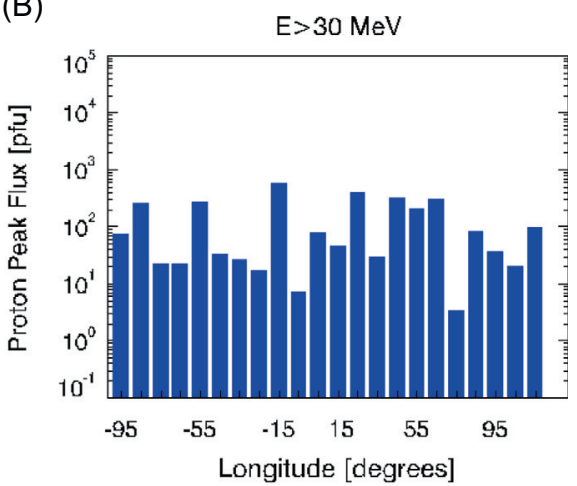

(D)

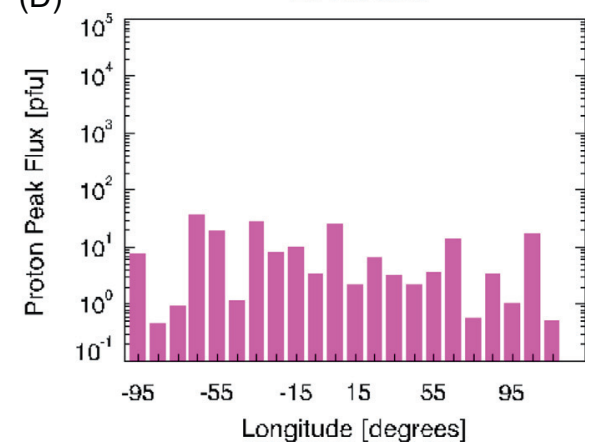

Fig. 11. The proton peak fluxes (in pfu) at several integral energies $(E>10 ;>30 ;>60$ and $>100 \mathrm{MeV})$, as a function of the longitude of the associated SF (in degrees). Each integral energy is color coded as in Figure 8.

of SEP events). There are 117 SEP events in common in our lists, distributed per group, as follows:

- Group 1 (Gl): Fe-rich, e-rich, p-poor: 12 events,

- Group 2 (G2): Fe-rich, p-rich: 37 events,

- Group 3 (G3): Fe-poor, p-rich, no shock passage: 13 events,

- Group 4 (G4): Fe-poor, p-rich, shock passage: 26 events,

- Group 5 (G5): slow rise, Fe-poor, peak at the shock passage: 20 events.

There were also nine events in common that are not included in any of the above groups. Those events have few ions or are preceded by another event producing a high background, according to Cane et al. (2010).

During the SOHO era (1997-2013), there are 174 SEP events reported in the SEP part of our database. 1/174 $(0.5 \%)$ has no identification of a parent solar event, 22/174 $(12.8 \%)$ are associated with a CME and not to a SF, $7 / 174(4 \%)$ are associated with a SF and not to a CME and $144 / 174(82.7 \%)$ are associated with both SFs and CMEs. It seems that the occurrence of an SEP event is related to both SFs and CMEs on a, roughly, equal basis. In order to test this we examine element abundances, electron measurements, radio burst data, the timing with respect to the Neupert effect and the location of the SFs related to the SEP events.

\subsection{Element abundances}

As a first step, we used element abundances data to shed light on the role of SFs and CME-driven shocks in the acceleration and release processes of SEP events. We utilized data from the Ultra Low Energy Spectrometer (ULEIS; Mason et al. 1998) and the Solar Isotope Spectrometer (SIS; Stone et al. 1998b) onboard the Advanced Composition Explorer (ACE; Stone et al. 1998a). In particular, the Fe and O intensities as well as their corresponding ratio were used to distinguish impulsive SEP events rather than ${ }^{3} \mathrm{He}$, since: [a] the threshold of ${ }^{3} \mathrm{He}$ in the SIS instrument is too high (Cane et al. 2010) and [b] the corresponding ratio of ${ }^{3} \mathrm{He} /{ }^{4} \mathrm{He}$ is "poorly" defined (Reames 2015). Similar to Cane et al. (2010), we inspected the time profiles of $\mathrm{Fe}$ and $\mathrm{O}$ intensities for all 117 SEP events and calculated the corresponding $\mathrm{Fe} / \mathrm{O}$ ratio $(0.32-0.64$, $34 \mathrm{MeV} /$ nucleon). Furthermore, we expanded our search up until the end of our catalogue (year 2013), adding identifications for another 12 SEP events. Therefore, a total of 120 SEP events (108 by Cane et al. 2010 plus 12) were distributed among the different groups, as follows: G1-13/120; G2-44/ 120; G3-15/120; G4-28/120 and G5-20/120.

Figure 16 illustrates the $\mathrm{Fe} / \mathrm{O}$ ratio at $\sim 34 \mathrm{MeV} / \mathrm{n}$ against that at $0.32-0.64 \mathrm{MeV} / \mathrm{n}$. The figure includes $57 \mathrm{SEP}$ events from 1998 to 2012 from $G 1$ to G5. This number corresponds to the SEP events that extended up to this high energy for ACE/SIS recordings. The ratios are for the early intensities as described in Cane et al. (2010). It can be seen that there are clear tendencies for the SEP events in the different groups to appear in the different parts of the scatter plot. The average SEP abundance ratio of 0.134 for gradual SEP events (Reames $1995)$ is indicated on each axis. The composition appears to be Fe-rich, at both low and higher energies. Note that for all but eight events (out of the 57), the $\mathrm{Fe} / \mathrm{O}$ is greater than 0.134 at low energies. In addition to the supply of Fe-rich suprathermals at impulsive SEP events, it seems that the material from gradual events is also Fe-rich. Furthermore, it seems that G2 events are all in the upper right part, i.e. those identified to have high ratios at high energies, have high ratios at low 
(A)

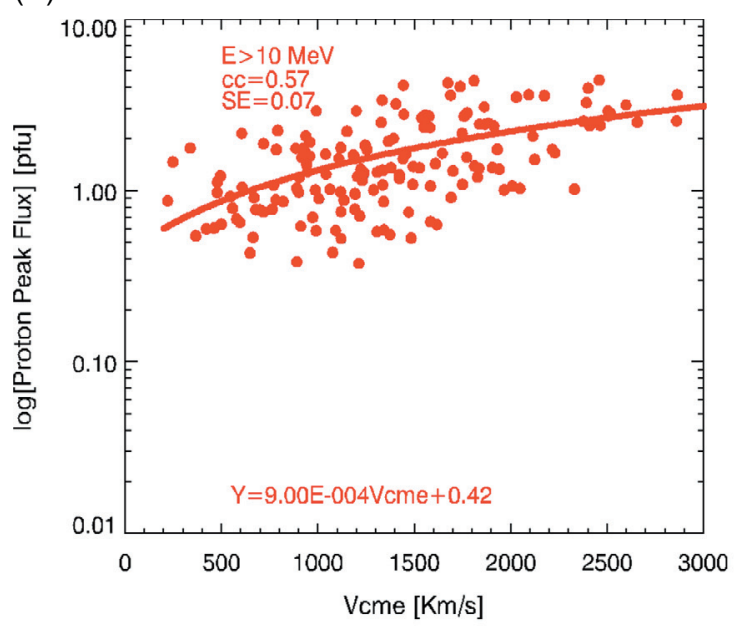

(C)

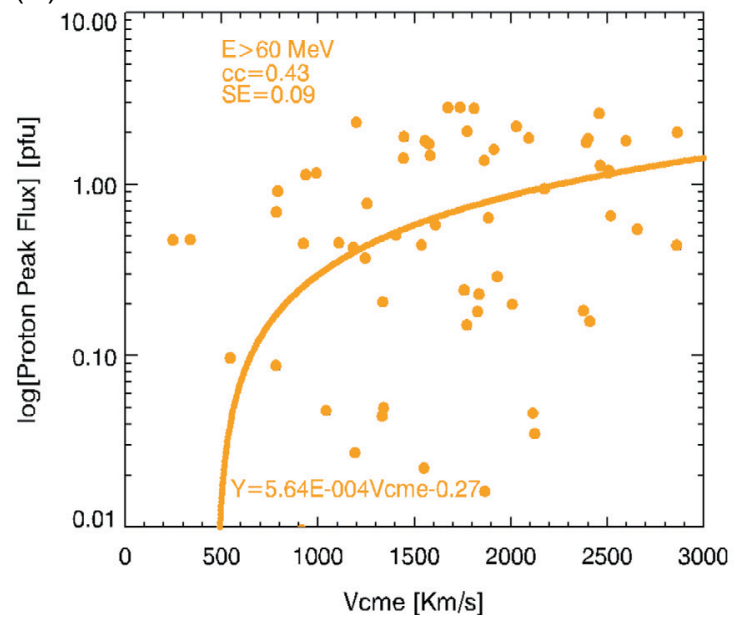

(B)

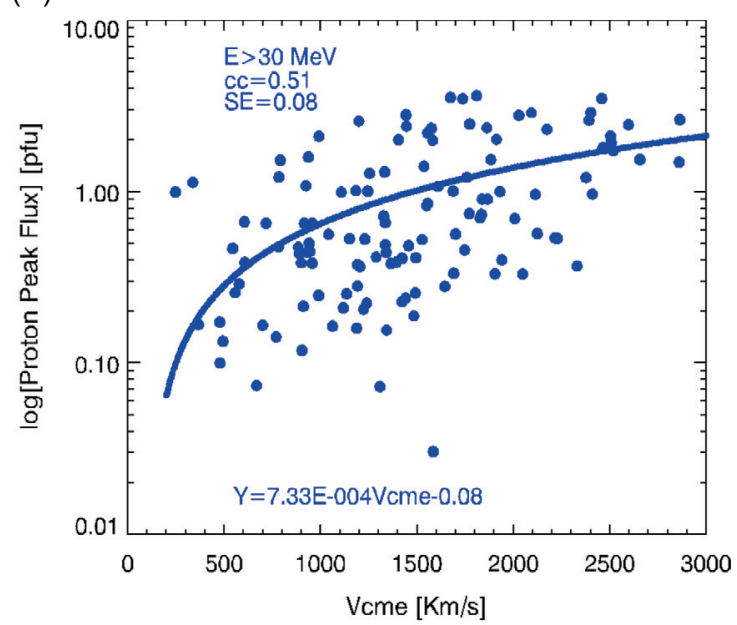

(D)

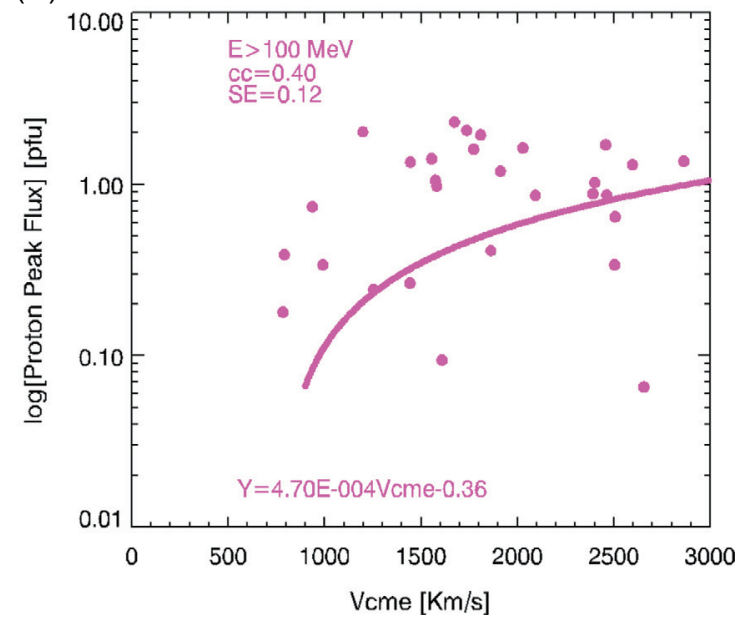

Fig. 12. The logarithm of the proton peak fluxes (in pfu) at several integral energies $(E>10[\mathrm{~A}] ;>30[\mathrm{~B}] ;>60[\mathrm{C}]$ and $>100 \mathrm{MeV}[\mathrm{D}])$ as a function of the plane-of-sky speed for the CMEs associated with SEP events in the SEP part of the database. The lines at each panel represent the linear fit to the data. Each panel, further, presents the correlation coefficient $(c c)$, the respective standard error $(S E)$ and the linear fit to the data.

energies as well. On the other hand, G4 events, i.e. the Fe-poor at high energies, can be Fe-rich at lower energies.

\subsection{Electron measurements}

Next, in order to complement our analysis on the proton recordings of the SEP events, we examined the electron data recorded onboard ACE/Electron Proton and Alpha Monitor (EPAM; Gold et al. 1998), from 1997 to 2013. In particular, we made use of the deflected electron (DE) recordings, at four energy channels ranging from 0.038 to $0.315 \mathrm{MeV}$ made available from the SEPServer. ${ }^{14}$ The parallel scanning of high time resolution (1 min) electron recordings from EPAM showed that the vast majority of the SEP events of our catalogue within the SOHO era (167/174 events, 96\%) also presented electron signatures. $e / p$ ratios defined as the ratio of the peak intensities of $\approx 0.24 \mathrm{MeV}$ electrons (mean energy of DE4)-P4 GOES/ EPS protons (mean energy of $24.5 \mathrm{MeV}$ ) show a continuum of values, with no distinct groups of relatively electron-rich and electron-poor events, in line with the findings of Cane et al. (2010). We have chosen to use a criterion of $e / p \geq 7.5 \times 10^{4}$, which would just exclude the event no. 146 of our catalogue from group 1, similar to Cane et al. (2010).

\footnotetext{
$\overline{14} \mathrm{http} / / /$ server.sepserver.eu
}

\subsection{Radio bursts}

Subsequently, we turned to radio data. There are 144/174 (83\%) SEP events for which we identified type III bursts. Furthermore, 129/174 (74\%) SEP events present clear signatures of shocks (e.g., type II bursts), while another 10/174 (5\%) events present indications of type II bursts. There are $127 / 174(73 \%)$ SEP events that have both type III and type II radio bursts. Finally, 62/174 (35\%) SEP events are associated with type IV radio bursts with an additional 13/174 (7\%) events, also, most probably related to type IV radio bursts.

First we subtracted the onset time of the type III burst $\left(t_{\text {onsettIII }}\right)$ from the time of the peak SF flux $\left(t_{\text {peak }_{\text {flare }}}\right)$ and obtained $\Delta t_{1}=t_{\text {onset }_{\text {III }}}-t_{\text {peak }_{\text {fare }}}$ which resulted in the distribution presented in Figure 17. Note that five events were discarded from the distribution since those provided a timing that seemed unphysical (i.e. $\Delta t_{1} \geq 5 \mathrm{~h}$ ). The ordinate presents the number of the events, while the abscissa gives the time difference obtained by the subtraction $\left(\Delta t_{1}\right)$ in minutes. Events to the left of the vertical dashed line had associated type III bursts that started only before the maximum of the flare intensity was achieved (i.e., $\Delta t_{1}<0$ ), whereas events to the right of the vertical dashed line had associated type III bursts that started after the SF maximum (i.e. $\Delta t_{1}>0$ ). Second, we applied the 
(A)
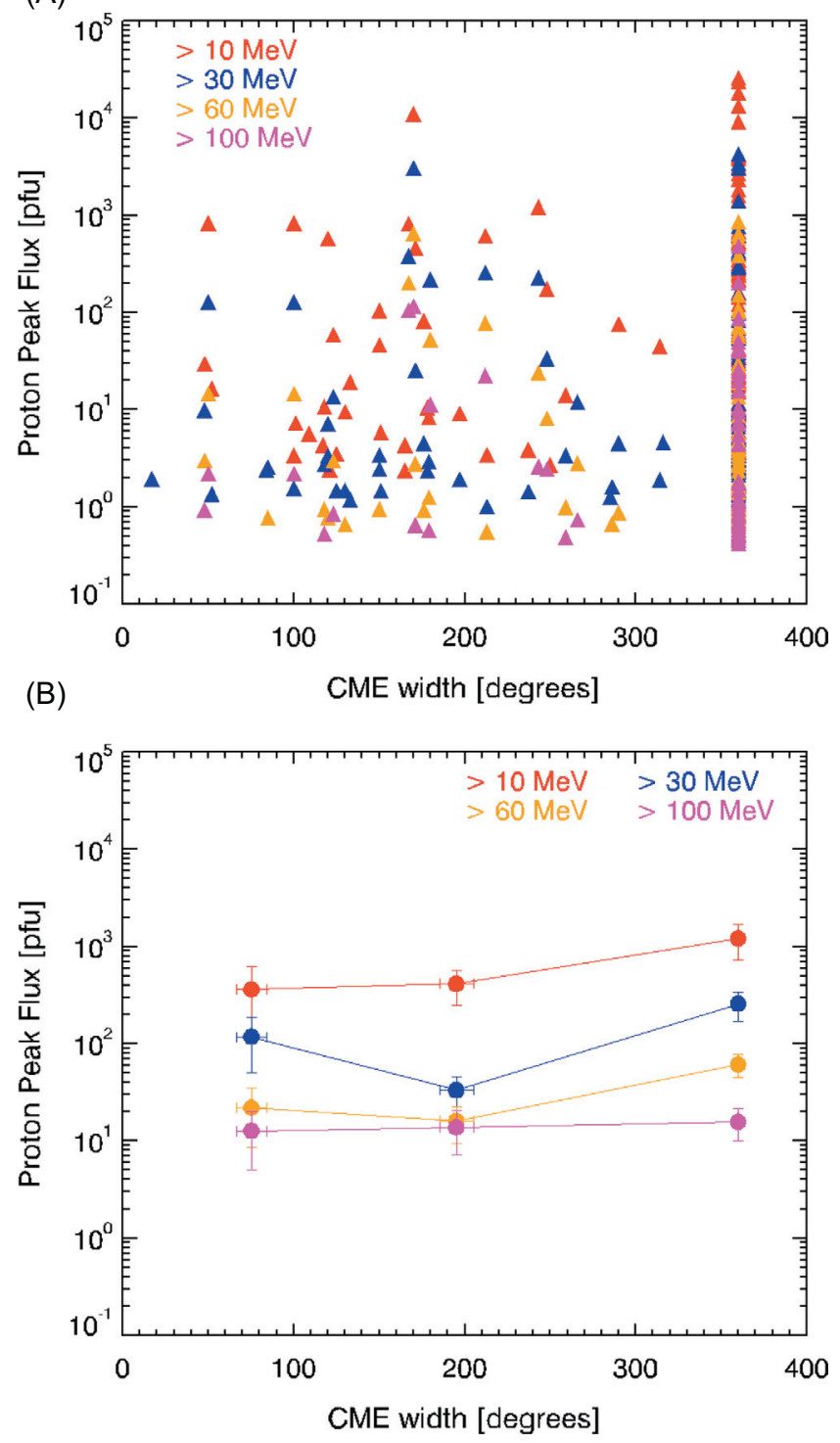

Fig. 13. (A) Scatter plot of the proton peak fluxes (in pfu) at several integral energies $(E>10 ; 30 ; 60$ and $100 \mathrm{MeV})$ as a function of the CME width (in degrees) for the CMEs associated with SEP events in the SEP part of the database. (B) Mean values of the proton peak flux (in pfu) and their respective standard deviation of the mean, as a function of the CME width (in degrees). Each integral energy is color coded as in Figure 8.

grouping of Cane et al. (2010) (e.g., G1-G5) and produced bar plots (Fig. 18) for the SEP events per group, against the relative timing $\Delta t_{1}$. One event that was included in the G5 group had an unphysical $\Delta t_{1}$ and thus was discarded from the analysis (hence, for G5-19/120 events were used). Following a counterclockwise direction, the first panel on the top left corresponds to G1 (Fe-rich, e-rich and p-poor) SEP events, labelled with red colour. The following panel corresponds to G2 (Fe-rich, p-rich) SEP events, labelled with orange colour. Under it the following panel corresponds to G3 (p-rich, Fe-poor, no shock) SEP events, labelled with blue colour. The next panel in the counterclockwise direction corresponds to G4 (p-rich, Fe-poor, shock) SEP events, labelled with magenta colour and right above it the last plot depicts the SEP events of G5 (slow rise, peak at the shock) (cyan colour).
It appears (Fig. 17) that the start of the type III burst most often (128/139 events; 92\%) precedes the maximum of the SF. Furthermore, the analysis of the timing of $\Delta t_{1}$ per group (Fig. 18) verifies the previous result but at the same time provides useful insight. The events of group 1 (G1) are, by definition, Fe-rich, electron-rich and p-poor. All of the events of G1 are associated with type III radio bursts. Hence, most of them could be labelled as impulsive SEP events. At the other end, the events of groups 4 and $5^{15}$ (G4 and G5) are considered to be Fe-poor, p-rich and in essence are gradual SEP events. Figure 18 shows that for all groups (even for the gradual SEP events), the onset time of the identified type III burst was marked prior to the maximum of the SXR peak flux and thus during the impulsive phase of the associated SF. Additionally, the largest mean $\Delta t_{1}$ is marked for G2 $(-19.04 \pm 2.96 \mathrm{~min})$ and the shortest one for G3 $(-11.80 \pm$ $3.84 \mathrm{~min}$ ). Finally, there is only one event in each of the G1 and G3 groups, two events in G2 and three events in G4 that present a positive $\Delta t_{1}$, while all events of G5 have a negative $\Delta t_{1}$.

\subsection{The Neupert effect}

Moreover, we calculated the maximum in the derivative of the SXR flux, as well as the time that this was marked. In this part, we used the time of the maximum in order to test whether the release of the particles into open magnetic field lines is empirically associated with the maximum in the derivative of the SXR flux (i.e. Neupert effect).

The simultaneous observation of hard X-rays (HXR) and metric/decimetric radio emission is commonplace during SFs and the relationship between type III bursts and HXR emissions has been studied for many years (Reid \& Ratcliffe 2014). Bursts of type III radio emissions have been found to temporally correlate with bursts in HXRs (Pick \& Vilmer 2008). In this part of the study, we applied the grouping of the SEP events (i.e. G1-G5) and performed a timing analysis, similar to Section 4.3. We first establish the time difference $\Delta t_{2}=t_{\text {onset }}$ III $_{\text {Newpert }}$, where $t_{\text {Neupert }}$ is the time of maximum of the derivative of the SXR flux. Figure 19 depicts $\Delta t_{2}$ for each group. Furthermore, it was interesting to see if the impulsive SEP events have the shorter time difference, as opposite to the gradual SEP events. Our results in Figure 19 show that on average, the type III burst falls very close to the derivative of the SXR flux in all groups. The shorter mean time difference is marked for G1 (Fe-rich, e-rich and p-poor; $-1.69 \pm 3.34$ ) and the larger one for G5 (slow rise, peak at the shock passage; $-8.47 \pm 4.08)$. Moreover, there are only four events $(4 / 13$, $31 \%)$ in G1 that have an absolute $\Delta t_{2} \geq 4 \mathrm{~min}$. This percentage is $39 \%$ (17/44 events) for G2, $60 \%$ (9/15) for G3, $57 \%$ (16/28) for $\mathrm{G} 4$ and $53 \%(10 / 19)$ for $\mathrm{G} 5$.

\subsection{Location of the SEP event related solar flares}

Figure 20 shows the distribution of the flare latitude with respect to the longitudinal distance of the solar flare from the footpoint of the Parker spiral leading to the observing point $(\Delta \Phi)$, as obtained from the solar-wind speed observed during the SEP event onset (Vainio et al. 2013). In particular, the equation for the solar longitude of the connection point (i.e. Earth) is (Nolte \& Roelof 1973):

\footnotetext{
${ }^{15}$ Note that we currently use $19 / 20$ G5 events. This is because there was one event in this group for which $\Delta t_{1}>400 \mathrm{~min}$ and thus was dropped from the analysis.
} 
(A)

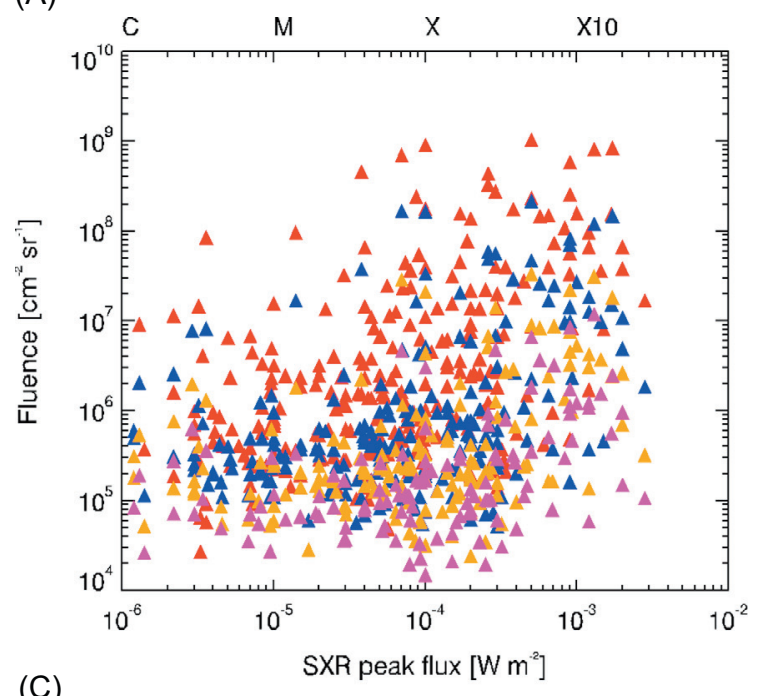

(C)

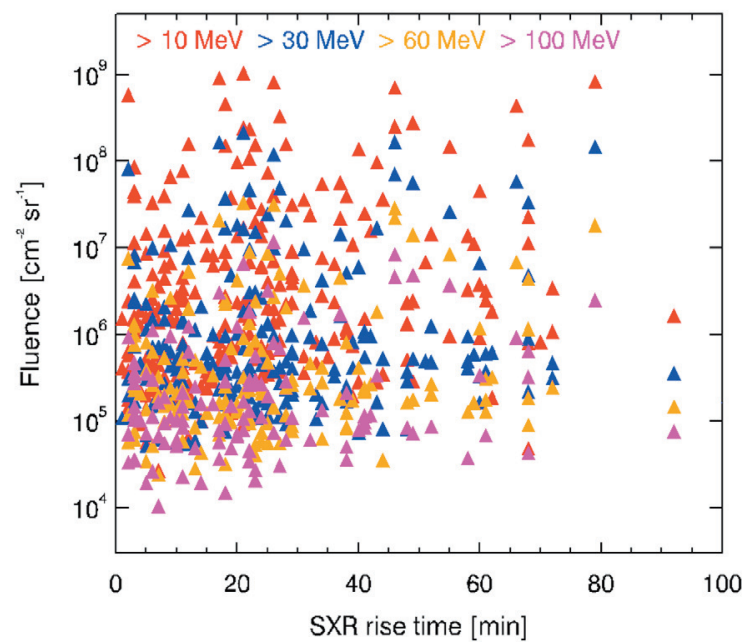

(B)

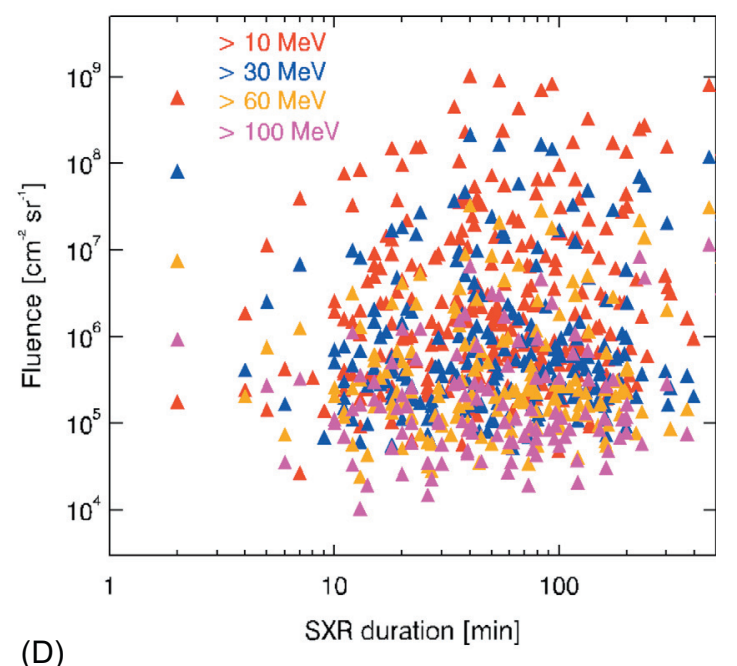

(D)

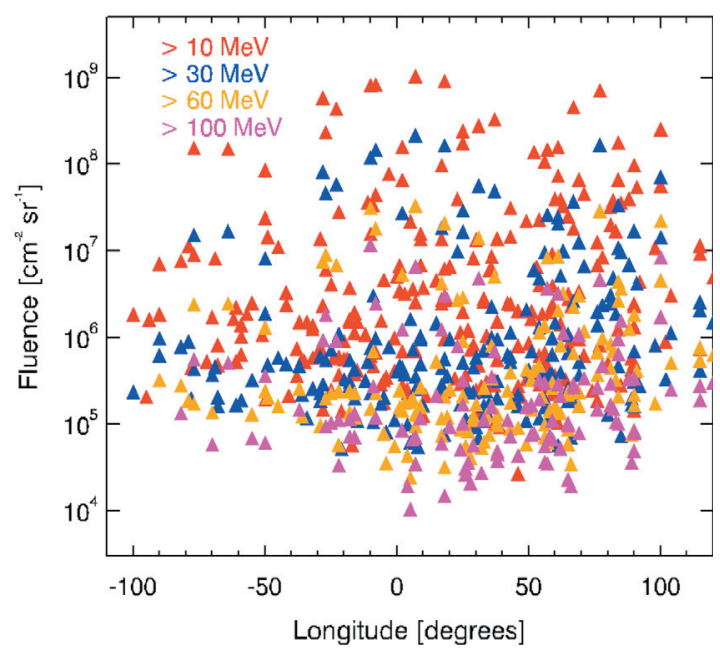

Fig. 14. The fluence (in $\mathrm{cm}^{-2} \mathrm{sr}^{-1}$ ) of the SEP events in the SEP part of the database for various integral energies (>10;30;60 and $\left.100 \mathrm{MeV}\right)$ as a function of the SXR peak flux (in $\mathrm{W} / \mathrm{m}^{2}$ ) (A); the duration of the associated parent solar flares (in minutes) (B); the rise time of the associated parent solar flares (in minutes) (C) and the longitude of the parent solar flare (in $\mathrm{W} / \mathrm{m}^{2}$ ) (D). (A) Incorporates the class, i.e. C, M, X and X10, of solar flares on its top part.

$$
\phi_{0}=\frac{\omega r}{u_{\mathrm{sw}}}\left(1-\frac{R_{S}}{r}\right)
$$

$r$ being the distance from the Sun centre to the connected point (in our case, $212 R_{\odot}$ for the Sun-Earth L1 point), $\omega$, the angular speed of the solar rotation (i.e. the equatorial solar rotation period of 24.47 days or $\omega=2.97 \times 10^{6}$ ) $\left.\mathrm{rad} / \mathrm{sec}\right), \quad R_{\mathrm{s}}=2.5 R_{\odot}$, the heliocentric distance beyond which the magnetic field is assumed to have the form of the Parker spiral, and $u_{\mathrm{sw}}$ the radial solar-wind speed, assumed constant in space. For the solar-wind speed, we used data from ACE/Solar Wind Electron Proton and Alpha Monitor (SWEPAM; McComas et al. 1998). ${ }^{16}$ Furthermore, the longitudinal distance of the solar flare from the footpoint of the Parker spiral leading to L1 was defined as: $\Delta \Phi=\phi_{\text {flare }}-\phi_{0}$.

Figure 20 depicts the groups G1-G5 in a colour code. For G1 (Fe-rich, e-rich, p-poor) SEP events (red colour),

\footnotetext{
${ }^{16} \mathrm{http}: / /$ www.srl.caltech.edu/ACE/ASC/level2/lvl2DATA_SWEPAM. $\mathrm{html}$
}

it seems that most of the events fall within a cone of approximately $\pm 50^{\circ}$ around the flare site, hereafter cone of observations, $(9 / 12,75 \%)$. However, there are a few exceptions $(3 / 12,25 \%)$ where G1 SEP events are visible up to an angular distance of $\sim 150^{\circ}$. The majority of the events of the G2 group (Fe-rich, p-rich) (orange colour) lie within the cone of observations $(26 / 37,70 \%)$, while several of them $(11 / 37,30 \%)$ are outside the cone, also extending up to $\sim 150^{\circ}$. Additionally, G3 (p-rich, Fe-poor, no shock) SEP events (blue colour) are mostly present within the cone of observations $(9 / 13,69 \%)$, with an extension of up to $\sim 80^{\circ}$. Moreover, almost all (26/27, 96\%) of the G4 (p-rich, Fe-poor, shock) SEP events (magenta colour) are within the cone of observations, with one event $(1 / 27,4 \%)$ extending to $90^{\circ}$. Finally, the G5 (slow rise, peak at the shock) SEP events (cyan colour) are also present within the cone $(16 / 20,80 \%)$, with several other events $(4 / 20,20 \%)$ extending up to $\sim 130^{\circ}$. As a result, a significant part of the coronal propagation is generally limited to a cone of approximately $\pm 50^{\circ}$ around the flare site for every group (i.e. G1-G5) of the SEP events. Additionally, the SEP events that fall into groups G2 and G5 extend up to $-150^{\circ}$. 
(A)
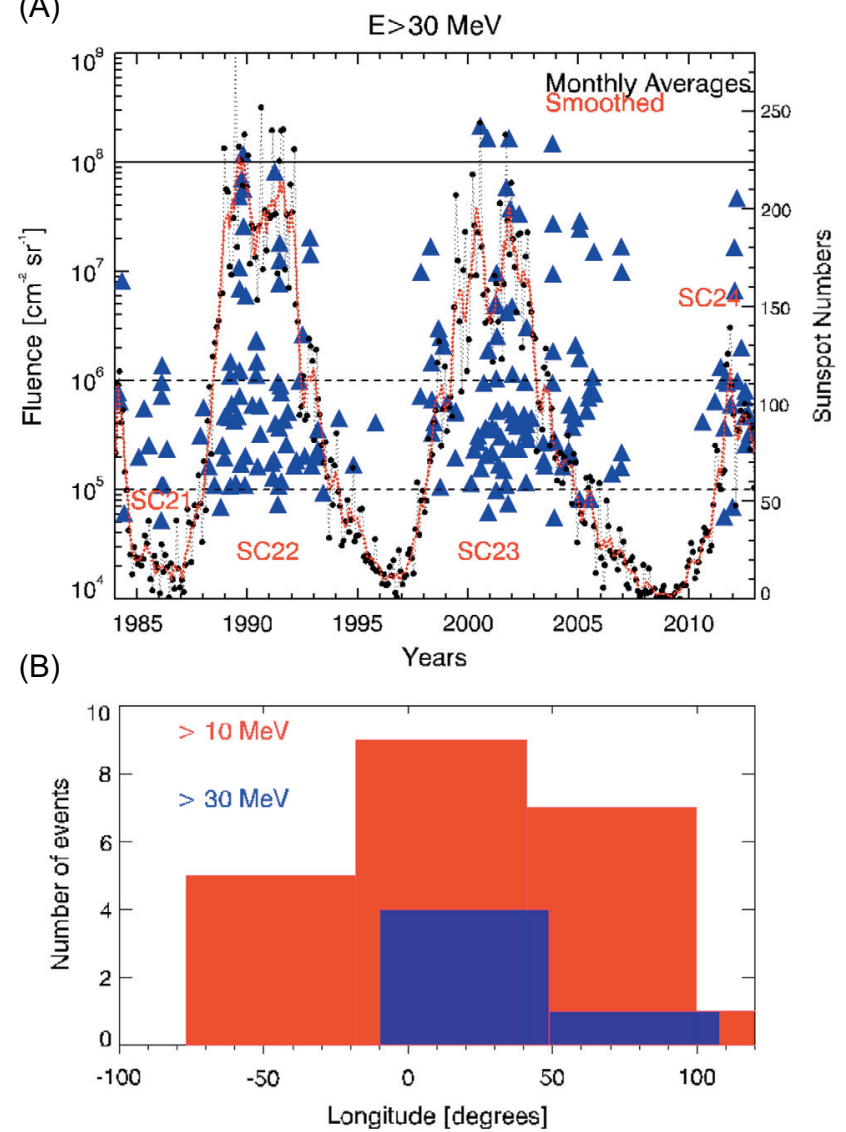

Fig. 15. (A) The fluence of the SEP events at $E>30 \mathrm{MeV}$ observed from 1984 to 2013 as a function of time. The dashed horizontal lines indicate the zone of the most commonly identified fluence values that falls between $10^{5}$ and $10^{6} \mathrm{~cm}^{-2} \mathrm{sr}^{-1}$. The solid horizontal black line marks an extreme fluence level at $10^{8} \mathrm{~cm}^{-2} \mathrm{sr}^{-1}$. The black filled circles correspond to the monthly averaged sunspot numbers and the red smoothed curve depicts the evolution of the solar cycles from 19842013. Each solar cycle is marked on the plot. (B) Distribution of the SEP events with fluence $\geq 10^{8} \mathrm{~cm}^{-2} \mathrm{sr}^{-1}$ at an integral energy of $E>10 \mathrm{MeV}$ (red color) and $>30 \mathrm{MeV}$ (blue color) as a function of the longitude of the associated solar flares (in degrees).

\subsection{Dependencies}

\subsubsection{Correlation coefficients}

Table 4 presents the Pearson's correlation coefficients between the logarithm of the proton peak fluxes at $E>10 ;>30,>60$ and $>100 \mathrm{MeV}$, the logarithm of the fluence at the same energies and the basic characteristics of the parent solar events: SFs and CMEs parameters - logarithm of the SXR peak flux, (log SXRs); the SXR fluence, $\left(I_{\mathrm{SXR}}\right)$; the maximum flux value of the time derivative of the SXRs (i.e. "Neupert effect") $\left(N_{\mathrm{e}}\right)$; longitude of the associated flare, (Lon); flare rise time, (RT); flare duration, $(D)$; velocity of the CME, $(u)$ and size of the CME, $(s)$. The number of pairs for the calculation of each coefficient is presented in parentheses next to the coefficients at each case. The confidence interval around a Pearson's coefficient is based on the so-called Fisher's $r$-to- $z$ transformation (Fisher 1921). For a sample of $N X$-Y pairs with

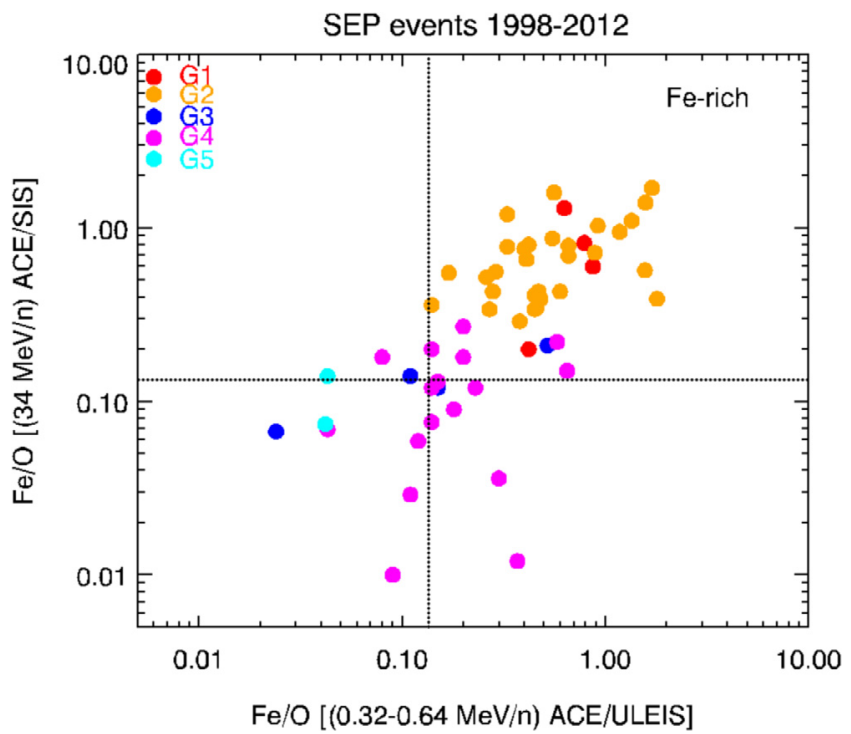

Fig. 16. The $\mathrm{Fe} / \mathrm{O}$ ratio at $34 \mathrm{MeV} / \mathrm{n}$ against that at 0.32 $0.64 \mathrm{MeV} / \mathrm{n}$ for the SEP events from 1998-2012. The dashed black lines indicate the average $\mathrm{Fe} / \mathrm{O}$ value of 0.134 (Reames 1995). The $34 \mathrm{MeV} / \mathrm{n}$ data are from ACE/SIS and the $0.32-0.64 \mathrm{MeV} / \mathrm{n}$ data from ACE/ULEIS. The five groups, i.e. G1-G5, as defined by Cane et al. (2010), are color coded on the plot. The events attributed to each group follow the same color code. The plot extends from 1998 to 2012. There are two events in our catalogue, identified in 1997 and another two events in 2013. However, in the first case ACE/ ULEIS presented data gaps while in the second one, there is a data gap of Fe at ACE/SIS at $34 \mathrm{MeV} / \mathrm{n}$.

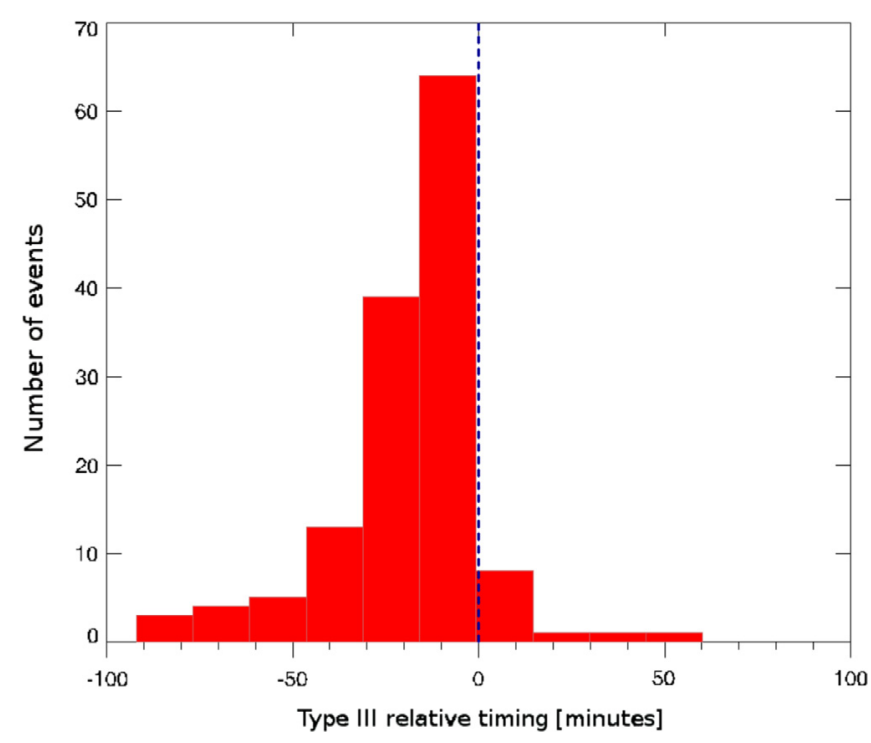

Fig. 17. Distribution of the SEP events with respect to $\Delta t_{1}=t_{\text {onset III }_{\text {II }}}-t_{\text {peak }_{\text {flare }}}$. Events to the left of the vertical dashed blue line had associated type III events that started prior to the time of the SXR peak flux, while events to the right of the vertical dashed blue line had associated type III events that occurred after the time of the SXR peak flux.

a Pearson's coefficient of $r$, the following transformation applies:

$$
z=0.5 \ln \left(\frac{1+r}{1-r}\right)
$$


A. Papaioannou et al.: SEP events and their parent solar sources
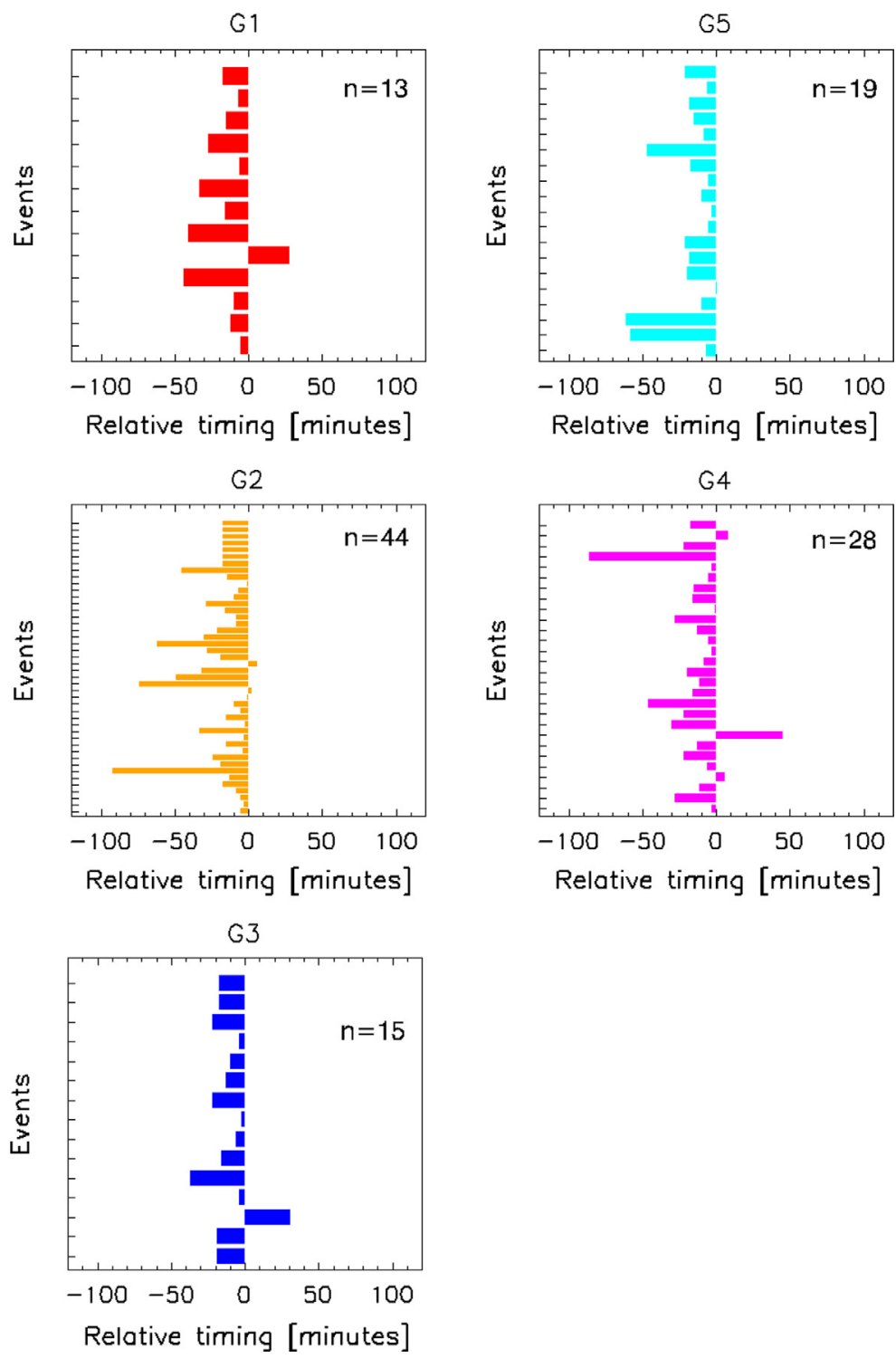

Fig. 18. Distribution of the SEP events with respect to $\Delta t_{1}=t_{\text {onset III }}-t_{\text {peak }}$ flare , for the five groups, i.e. G1-G5, as defined by Cane et al. (2010). Events with a negative $\Delta t_{1}$ had associated type III events that started prior to the time of SXR peak flux, while events with a positive $\Delta t_{1}$ had associated type III events that occurred after the time of the SXR peak flux.

For the transformed $z$, the approximate variance $V(z)=1 /$ $(N-3)$ and the corresponding standard deviation $\sigma_{z}=1 / \sqrt{N-3}$ are independent of the correlation. Furthermore, even the distribution of $z$ is not strictly normal; it tends to be normal rapidly as the sample size increases for any value of $r$ (Shen \& Lu 2006). The confidence intervals (CI) of a correlation coefficient $r$ are computed based on the sample mean and its standard deviation. Since the variance is dependent on both sample size and $r$ size, the CI cannot be computed directly. However, Fisher transformation makes it possible to calculate the CI indirectly. In particular, the upper and lower CI limits of the Pearson's correlation $r$ are defined as: $\mathrm{z}_{(u / l)}=z \pm z_{1-\alpha / 2} \sigma_{z}$ with $z_{1-\alpha / 2}=100(1-a / 2)$ being the percentage point of the standard normal distribution. $\alpha$ stands for the significance level and thus the probability of error (Shen \& Lu 2006). Therefore, when choosing the desired CI (e.g., 95\% corresponding to $\alpha=0.05$ ) it is possible to mark the upper and lower limits of the correlation for the specified CI. Under each correlation coefficient of
Table 4 we present the upper and lower limits for a $\mathrm{CI}=90 \%$. In addition, we calculated the $p$-values ( $p$-value is the probability of obtaining a result as extreme as, or more extreme than, the result actually obtained when the null hypothesis is true) for each correlation coefficient (Fenton \& Neil 2012) and all $p$-values are included in Table 4. The most significant correlation coefficients are emphasized with bold fonts. The number in parentheses corresponds to the number of events used for the establishment of the correlation coefficients, their upper and lower CI and the $p$-value.

It appears that the correlation of the proton peak flux with the velocity of the CME is clearly the most prominent one, with a tendency to decrease when moving to higher energies. The second most significant correlation coefficient is the one derived from the proton peak fluxes and the SXR peak flux. This coefficient seems to remain fairly stable at all energies. The correlation between the proton peak flux and the SXR fluence presents a gradual decrease from $E>10 \mathrm{MeV}$ to $E>100 \mathrm{MeV}$. The calculated fluence of the SEP events 

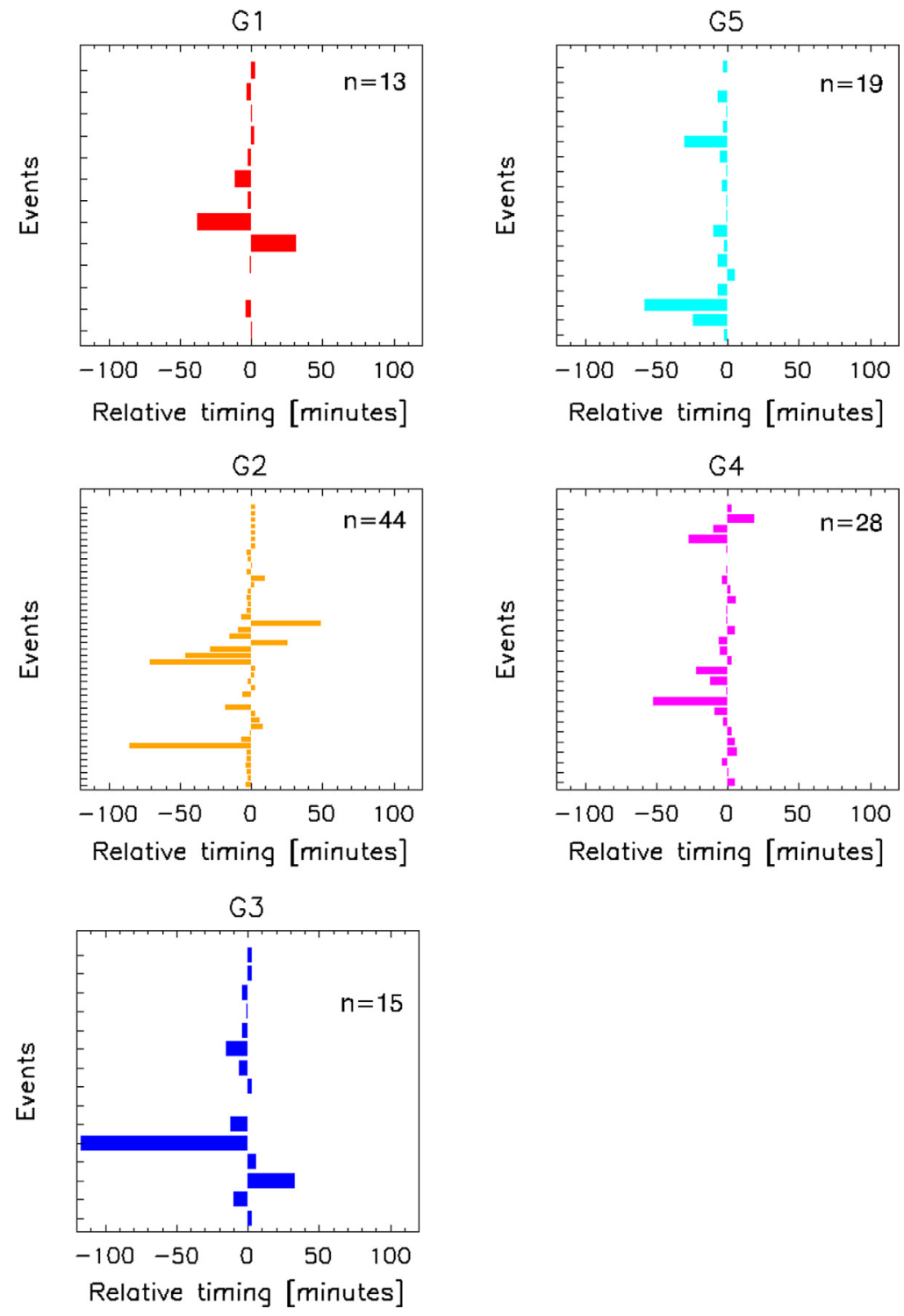

Fig. 19. Distribution of the SEP events with respect to $\Delta t_{2}=t_{\text {onset }}-t$, for the five groups, i.e. G1-G5, as defined by Cane et al. (2010). Events with a positive $\Delta t_{2}$ had associated time of maximum of the derivative of the SXRs that started prior to the onset time of the type III bursts, while events with a negative $\Delta t_{2}$ had associated time of maximum of the derivative of the SXRs that occurred after the onset time of the type III bursts.

presents a similar tendency in the correlations (see Tab. 4), with the most prominent one derived for the fluence of the SEP events and the velocity of the CME $(0.60$ for $E>10 \mathrm{MeV})$, followed by the one obtained for the SXR peak flux $(0.48$ for $E>10 \mathrm{MeV})$ and for the SXR fluence $(0.49$ for $E>10 \mathrm{MeV})$. Again, SFs seem to have a stable role in the achieved fluence at all energies while the velocity of the CME presents slightly higher coefficients with the same tendency as with the proton peak fluxes. Finally, the correlation of the SXR fluence to the proton peak flux slightly decreases with the increasing integral energy. However, the correlation of the SXR fluence to the fluence of the SEP events seems to be stronger at low energies. Finally, the duration, rise time, longitude and the time derivative of the SXR flare are not significantly correlated either to the proton peak flux or to the fluence of the SEP events at all energies, i.e. none of these parameters alone seem to be critical for the severity of an SEP event. Hence, an analysis of properly

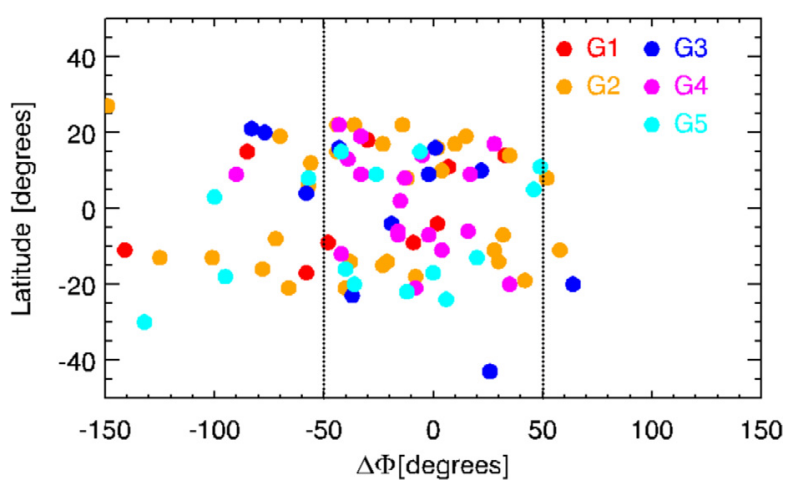

Fig. 20. Distribution of the flare locations with respect to the longitudinal distance of the solar flare from the footpoint of the Parker spiral leading to L1 point $(\Delta \Phi)$. The dashed lines indicate a "cone of observations" within $\left[-50^{\circ}, 50^{\circ}\right]$. The five groups, i.e. G1-G5, are color coded on the plot. The events attributed to each group follow the same color code. 
A. Papaioannou et al.: SEP events and their parent solar sources

Table 4. Pearson's correlation coefficients between the logarithm of the proton peak fluxes $(E>10 ;>30 ;>60$ and $>100 \mathrm{MeV})$ (upper part of the Table), the logarithm of the fluence (lower part of the table) and the basic characteristics of the parent solar events: flare (CME) parameters - logarithm of the SXR peak flux, $\left(\log\right.$ SXRs); SXR fluence, $\left(I_{\mathrm{SXR}}\right)$; the maximum flux value of the time derivative of the SXRs (i.e. "Neupert effect") $\left(N_{e}\right)$; longitude of the associated flare, $(L o n)$; flare rise time, $(R T)$; flare duration, $(D)$; velocity of the CME, $(u)$ and the angular width of the CME, $(s)$. The number of pairs for the calculation of each coefficient are presented in the parenthesis next to the coefficient at each case. The most significant coefficients are emphasized with bold fonts.

\begin{tabular}{|c|c|c|c|c|c|c|c|c|}
\hline \multirow{2}{*}{$\begin{array}{l}\text { Peak flux } \log 10 \\
\text { ([pfu]) }\end{array}$} & \multicolumn{6}{|c|}{ Solar flare } & \multicolumn{2}{|c|}{ CME } \\
\hline & $\log [S X R s]$ & $I_{S X R}$ & $N$ & Lon & $R T$ & $D$ & $u$ & $s$ \\
\hline$E>10 \mathrm{MeV}$ & $0.49(284)$ & $0.47(146)$ & 0.23 (149) & $0.11(262)$ & 0.07 (279) & $0.13(280)$ & $\mathbf{0 . 5 7}(158)$ & $0.21(158)$ \\
\hline $90 \%$ CI & {$[0.42,0.55]$} & {$[0.37,0.56]$} & $\begin{array}{l}{[0.11} \\
0.34]\end{array}$ & $\begin{array}{l}{[0.02} \\
0.20]\end{array}$ & $\begin{array}{c}{[-0.017} \\
0.16]\end{array}$ & $\begin{array}{c}{[0.046} \\
0.22]\end{array}$ & {$[0.49,0.65]$} & $\begin{array}{c}{[0.09} \\
0.32]\end{array}$ \\
\hline$p$-value & $<1 \times 10^{-6}$ & $1.34 \times 10^{-9}$ & 0.005 & 0.072 & 0.234 & 0.024 & $3.55 \times 10^{-15}$ & 0.008 \\
\hline$E>30 \mathrm{MeV}$ & $\mathbf{0 . 4 7}(207)$ & $\mathbf{0 . 4 9}(119)$ & $0.25(121)$ & 0.22 (194) & 0.05 (204) & $0.09(205)$ & $\mathbf{0 . 5 1}(127)$ & 0.09 (127) \\
\hline $90 \%$ CI & {$[0.39,0.55]$} & {$[0.38,0.59]$} & $\begin{array}{c}{[0.12} \\
0.37]\end{array}$ & $\begin{array}{c}{[0.12} \\
0.32]\end{array}$ & $\begin{array}{c}{[-0.05} \\
0.15]\end{array}$ & $\begin{array}{c}{[-0.009} \\
0.19]\end{array}$ & {$[0.40,0.59]$} & $\begin{array}{c}{[-0.04} \\
0.22]\end{array}$ \\
\hline$p$-value & $5.28 \times 10^{-13}$ & $1.62 \times 10^{-8}$ & 0.006 & 0.0017 & 0.46 & 0.18 & $1.33 \times 10^{-9}$ & 0.30 \\
\hline$E>60 \mathrm{MeV}$ & $\mathbf{0 . 4 8}(157)$ & $0.44(88)$ & $0.11(89)$ & $0.23(147)$ & 0.07 (155) & 0.13 (157) & 0.44 (93) & $-0.01(93)$ \\
\hline $90 \%$ CI & {$[0.39,0.57]$} & {$[0.30,0.55]$} & $\begin{array}{c}{[-0.04} \\
0.27]\end{array}$ & $\begin{array}{l}{[0.11} \\
0.34]\end{array}$ & $\begin{array}{c}{[-0.05} \\
0.19]\end{array}$ & $\begin{array}{c}{[0.017,} \\
0.25]\end{array}$ & {$[0.30,0.55]$} & $\begin{array}{c}{[-0.166} \\
0.15]\end{array}$ \\
\hline$p$-value & $1.39 \times 10^{-10}$ & $2.21 \times 10^{-5}$ & 0.285 & 0.0045 & 0.39 & 0.09 & $1.63 \times 10^{-5}$ & 0.90 \\
\hline$E>100 \mathrm{MeV}$ & $\mathbf{0 . 4 9}(118)$ & $0.42(64)$ & $0.13(64)$ & 0.19 (112) & 0.18 (116) & 0.19 (118) & $\mathbf{0 . 4 0}(62)$ & $-0.01(62)$ \\
\hline $90 \%$ CI & {$[0.38,0.59]$} & {$[0.25,0.56]$} & $\begin{array}{c}{[-0.06} \\
0.30]\end{array}$ & $\begin{array}{c}{[0.05} \\
0.32]\end{array}$ & $\begin{array}{c}{[0.047} \\
0.31]\end{array}$ & $\begin{array}{c}{[0.05} \\
0.31]\end{array}$ & {$[0.23,0.55]$} & $\begin{array}{c}{[-0.20 .} \\
0.18]\end{array}$ \\
\hline$p$-value & $1.77 \times 10^{-8}$ & $<5 \times 10^{-4}$ & 0.308 & 0.044 & 0.048 & 0.042 & 0.001 & 0.911 \\
\hline $\begin{array}{c}\text { Fluence } \log 10 \\
\left(\left[\mathrm{~cm}^{-2} \mathrm{sr}^{-1}\right]\right)\end{array}$ & $\log [S X R s]$ & $I_{S X R}$ & $N$ & Lon & $R T$ & $D$ & $u$ & $s$ \\
\hline$E>10 \mathrm{MeV}$ & $0.48(284)$ & 0.49 (146) & 0.21 (149) & $\begin{array}{l}0.058 \\
(262)\end{array}$ & 0.08 (279) & $0.15(280)$ & $0.60(158)$ & $0.20(158)$ \\
\hline $90 \% \mathrm{CI}$ & {$[0.41,0.54]$} & {$[0.39,0.58]$} & $\begin{array}{l}{[0.09} \\
0.32]\end{array}$ & $\begin{array}{c}{[-0.03} \\
0.15]\end{array}$ & $\begin{array}{c}{[-0.007,} \\
0.19]\end{array}$ & $\begin{array}{l}{[0.06} \\
0.23]\end{array}$ & {$[0.52,0.67]$} & $\begin{array}{c}{[0.089,} \\
0.31]\end{array}$ \\
\hline$p$-value & $<1 \times 10^{-6}$ & $2.72 \times 10^{-10}$ & 0.008 & 0.347 & 0.177 & 0.014 & $<1 \times 10^{-6}$ & 0.0096 \\
\hline$E>30 \mathrm{MeV}$ & $\mathbf{0 . 4 2}(207)$ & $\mathbf{0 . 4 9}(119)$ & $0.20(121)$ & 0.13 (194) & $\begin{array}{l}0.098 \\
(204)\end{array}$ & 0.13 (205) & $\mathbf{0 . 5 3}(127)$ & $0.11(127)$ \\
\hline $90 \% \mathrm{CI}$ & {$[0.33,0.50]$} & {$[0.37,0.58]$} & $\begin{array}{l}{[0.06,} \\
0.32]\end{array}$ & $\begin{array}{c}{[0.027,} \\
0.23]\end{array}$ & $\begin{array}{c}{[-0.005} \\
0.20]\end{array}$ & $\begin{array}{l}{[0.03,} \\
0.23]\end{array}$ & {$[0.43,0.62]$} & $\begin{array}{c}{[-0.021} \\
0.24]\end{array}$ \\
\hline$p$-value & $2.72 \times 10^{-10}$ & $2.27 \times 10^{-8}$ & 0.03 & 0.065 & 0.163 & 0.059 & $1.52 \times 10^{-10}$ & 0.217 \\
\hline$E>60 \mathrm{MeV}$ & $\mathbf{0 . 4 1}(157)$ & $\mathbf{0 . 3 5}(88)$ & $0.08(89)$ & $0.11(147)$ & $0.10(155)$ & $0.17(157)$ & $\mathbf{0 . 4 5}(93)$ & $0.03(93)$ \\
\hline $90 \%$ CI & {$[0.31,0.51]$} & {$[0.21,0.48]$} & $\begin{array}{c}{[-0.08} \\
0.23]\end{array}$ & $\begin{array}{c}{[-0.01} \\
0.23]\end{array}$ & $\begin{array}{c}{[-0.017} \\
0.22]\end{array}$ & $\begin{array}{c}{[0.06} \\
0.29]\end{array}$ & {$[0.32,0.56]$} & $\begin{array}{c}{[-0.13} \\
0.18]\end{array}$ \\
\hline$p$-value & $6.74 \times 10^{-8}$ & $<1 \times 10^{-3}$ & 0.47 & 0.184 & 0.207 & 0.0288 & $6.7 \times 10^{-6}$ & 0.80 \\
\hline$E>100 \mathrm{MeV}$ & $\mathbf{0 . 4 2}(118)$ & $\mathbf{0 . 3 8}(64)$ & $0.13(64)$ & 0.07 (112) & $0.20(116)$ & $0.25(118)$ & $\mathbf{0 . 4 6}(62)$ & $0.04(62)$ \\
\hline $90 \%$ CI & {$[0.30,0.53]$} & {$[0.20,0.57]$} & $\begin{array}{c}{[-0.064,} \\
0.30]\end{array}$ & $\begin{array}{c}{[-0.07} \\
0.21]\end{array}$ & $\begin{array}{c}{[0.06} \\
0.33]\end{array}$ & $\begin{array}{c}{[0.11} \\
0.37]\end{array}$ & {$[0.30,0.60]$} & $\begin{array}{c}{[-0.16} \\
0.22]\end{array}$ \\
\hline$p$-value & $2.4 \times 10^{-6}$ & $<1 \times 10^{-3}$ & 0.333 & 0.449 & 0.03 & 0.007 & $<1 \times 10^{-4}$ & 0.789 \\
\hline
\end{tabular}

combined pairs of parameters is performed in the following section.

\subsubsection{Dependence of SEP characteristics on pairs of parent solar source characteristics}

Since all of the aforementioned correlation coefficients are implemented on 2-D space (see Sect 4.6.1), the next step was to investigate the possible 3-D relationships among three numeric variables projected in two dimensions (2-D). Such an approach is in need of a complete, parametric space, as expressed by the basic characteristics of the database; see Table 4. Therefore, a total of $126 / 314$ SEP events presenting complete information - in terms of the parametric space - have been used in this part of the analysis. Contour plots, which depict the variation of the calculated logarithm of the proton peak flux at $E>10 \mathrm{MeV}$ (in pfu), that is considered as the "response", are plotted over the $Y X X$ space, which in turn is implemented by different pairs of variables/precursors. Identical plots were implemented also for the fluence of the SEP events at an integral energy of $E>10 \mathrm{MeV}$, leading to similar results and thus are not shown.

Figure 21A presents the variation of the logarithm of the proton peak flux as a function of the velocity of the CME, (u) and the logarithm of the SXR peak flux $(\log (\mathrm{SXRs}))$. It seems that intense proton peak fluxes $(\geq S 2)$ occur when the velocity of the CME ranges from $u>2400$, in $\mathrm{km} \mathrm{s}^{-1}$ and the associated solar flares are relatively strong, i.e. $\geq \mathrm{M} 2.0$. Furthermore, events that result in low proton peak fluxes $(<$ S1) are associated with CMEs with a velocity $u<500 \mathrm{~km} \mathrm{~s}^{-1}$ and extend from C3.0 to M9.0 solar flares. This means that strong solar flares and fast CMEs result in enhanced radiation storms, as opposite to weak solar flares and slow CMEs. Moreover, another area that concentrates enhanced proton peak fluxes $(\geq S 3)$ is identified at a CME velocity of $2000<u<2500$, in $\mathrm{km} \mathrm{s}^{-1}$ and a corresponding 
(A)

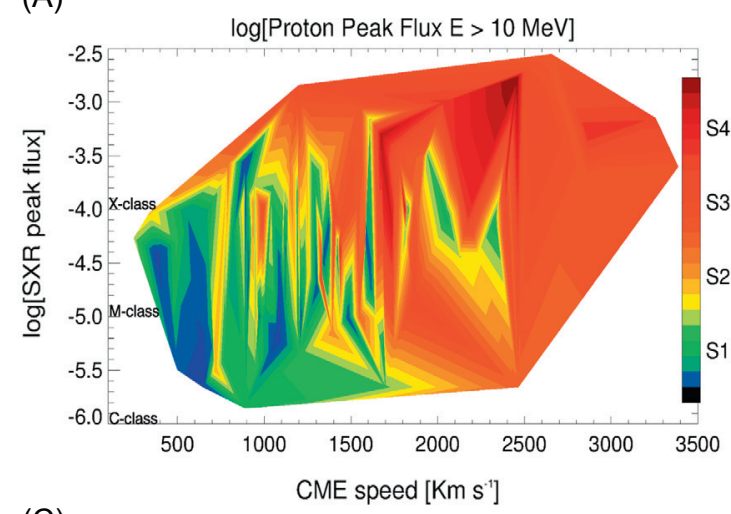

(C)

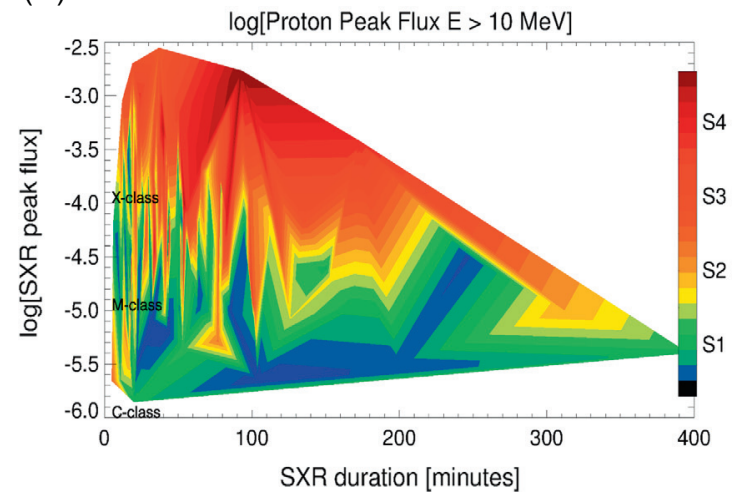

(B)

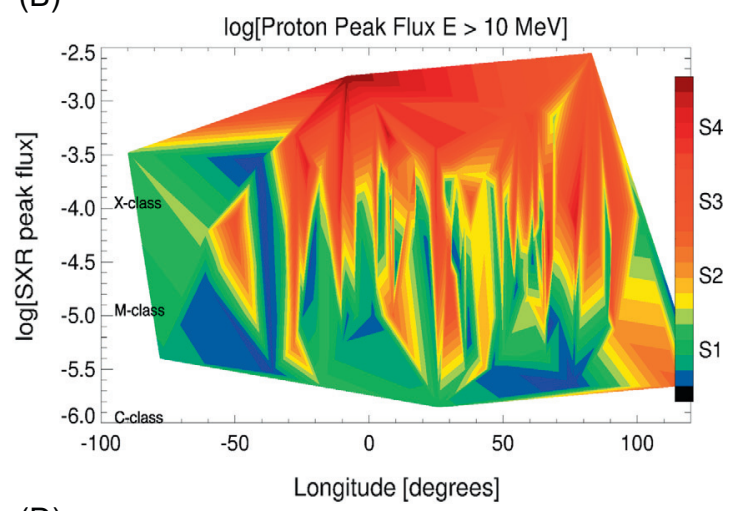

(D)

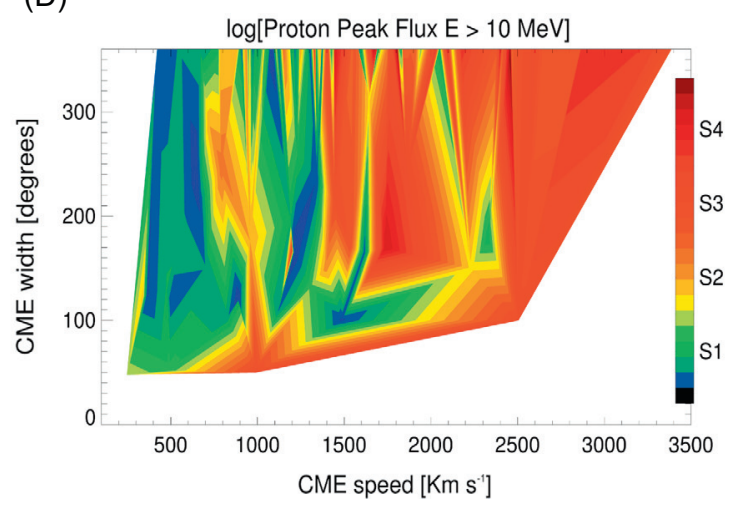

Fig. 21. Contour plots of the logarithm of the proton peak flux at $E>10 \mathrm{MeV}$ as a function of different pairs of variables. In particular, the variation of the logarithm of the proton peak flux at $E>10 \mathrm{MeV}$, as a function of the speed of the CME and the logarithm of the SXR peak flux is depicted at (A). (B) The variation, as a function of the longitude and the magnitude (SXR peak flux) of the parent solar flare. (C) The dependence of the logarithm of the proton peak flux at $E>10 \mathrm{MeV}$, as a function of the duration and the magnitude (e.g. SXR peak flux) of the parent solar flare and (D) presents the dependence, as a function of the speed and the width of the associated CME event. The color bar at each panel marks the different levels of radiation storms, as those are defined by NOAA, labelled with S1 to S4. See text for details.

range of the solar flare magnitude from M9.0 to X10.0. What is noteworthy, though, is a relatively narrow area of the velocity of the CME, ranging from $1600<u<1800$, in $\mathrm{km} \mathrm{s}^{-1}$ and the associated solar flares range, in magnitude, from M1.0 to X7.0 which also results in enhanced radiation levels $(\geq S 3)$. Both areas point to the conclusion that enhanced proton peak fluxes at $E>10 \mathrm{MeV}$ occur in concomitance with very fast CMEs and strong solar flares, which in turn constitute a direct danger for severe radiation storms.

Moreover, Figure 21B displays the variation of the logarithm of the proton peak flux as a function of the longitude of the associated solar flare and the logarithm of the SXR peak flux. One may notice the extension of the enhanced proton peak fluxes $(\geq S 3)$ over a broad longitudinal distribution for solar flares $\geq \mathrm{X} 4.0$. Nonetheless, $\geq \mathrm{M} 1.0 \mathrm{SFs}$ distributed from -60 to $100^{\circ}$ in longitude also lead to significant radiation storms. This result is consistent with the severe danger that stems from the SEP events that arise from the central part of the visible solar disk and are associated with CMEs, leading to a significant radiation risk, in agreement with the category of the interplanetary shock dominated events, as discussed earlier.

Furthermore, Figure 21C depicts the dependence of the logarithm of the proton peak flux at $E>10 \mathrm{MeV}$ as a function of the duration of the associated SF and the logarithm of the SXR peak flux. As it can be seen, enhanced radiation storms $(\geq$ S3) are marked for a SF of X5.0 or higher class and a SXR duration of $<60 \mathrm{~min}$. At the same time SFs with a
SXR duration of $\geq 60 \mathrm{~min}$ are associated with strong radiation storms ( $\geq \mathrm{S} 3)$ even for solar flares with a magnitude of M5.0. Nonetheless, all $\geq X 1.0$ solar flares lasting up to $200 \mathrm{~min}$ lead to enhanced radiation levels $(\geq \mathrm{S} 2)$.

Finally, Figure 21D presents the variation of the logarithm of the proton peak flux at $E>10 \mathrm{MeV}$ as a function of the velocity and the width of the associated CMEs. As it can be seen CMEs with a velocity of $u>2500 \mathrm{~km} \mathrm{~s}^{-1}$ will result in a significant radiation storm $(\geq \mathrm{S} 3)$ even for $\mathrm{CME}$ widths $\geq 80^{\circ}$. On the other hand, there are several more areas of enhanced radiation levels $(\geq \mathrm{S} 3)$ for velocities $u=1500$ $1700 \mathrm{~km} \mathrm{~s}^{-1}$ for CME widths $\geq 130^{\circ}$. Halo CMEs (with a width of $360^{\circ}$ ) associated with velocities $u>1400 \mathrm{~km} \mathrm{~s}^{-1}$ lead to radiation storms of $\geq \mathrm{S} 2$.

\section{Discussion and conclusions}

The motivation of the study was to extract empirical relations of SEP events to their parent solar sources and to explore further, the physical interpretations that have been proposed so far for the birth, acceleration and propagation of SEP events. Within the present study, we analysed the relationship between the proton peak fluxes and fluences for $E>10 ; 30 ; 60$ and $100 \mathrm{MeV}$ and the parameters that describe the parent solar activity from 1984 to 2013 . Our study included a total number of 314 SEP events. The solar parameters considered in this study are: the CME velocity, the peak SXR flare flux, the 
fluence and the location of the parent SF, the CME size expressed by its width, the duration and rise time of the SFs, the maximum of the derivative of the SXRs flux as well as its timing, the onset time of type III radio bursts, the occurrence (or not) of type II and type IV radio bursts, the element abundances and complementary electron recordings of the SEP events.

The main findings of this study are summarized as follows:

\subsection{A new catalogue of SEP events with solar associations}

We reported on the implementation of a coherent SEP event list based on GOES particle proton data, covering roughly three solar cycles from 1984 to 2013, including a total of 314 SEP events with their solar associations. For each of the events we have performed VDA, in conjunction with an extended literature survey and comparative observational identifications (see Fig. 3), in order to identify the possible release time range of the particles and correspondingly to be led to their parent solar event(s).

The new SEP events catalogue, presented in full in Table 2 (supplementary online material) of this paper, has four basic merits:

- It covers a large time span facilitating the exploitation of its contents for both scientific and forecasting/nowcasting purposes.

- It utilizes re-defined energies $\left(E_{\text {effective }}\right)$ for the VDA calculations, per energy channel.

- It provides re-checked and improved solar associations of the SEP events to their parent solar events.

- It is built upon a reference SEP event list, SEPEM, and thus can be considered as a complementary extension to an already standard SEP event list.

The implementation of this new database, the incorporation of the $E_{\text {effective }}$ in the VDA method and the cross-checking with several other available lists, allowed us to obtain careful reconciled associations of the SEP events to their parent solar sources covering a large time span. As a result, the SEP events list that was presented in this work can be considered as a standardized database that can further be used for both the testing of SEP events forecasting/nowcasting models and for the derivation of solid refined empirical physical relations that can be included in such forecasting efforts.

\subsection{A hybrid mixed CME-SF causation of SEP events?}

Concerning the origin of the particles that result in the recorded SEP events, the majority of the SEP events in our database are associated with both SFs and CMEs (208/314) (see Tab. 3, supplementary online material). One may also note that the lack of CMEs from 1984 to 1997 is mainly due to the absence of continuous CME monitoring and not because of the absence of CME events. Furthermore, we examined the abundance variations of 174 SEP events in our list from 1997 to 2013. Out of these, 120 events were divided into five groups based on the abundances and the particle profiles (Cane et al. 2010). The most important result is that there seems to be a continuum of event properties. For example, the events of group 1 (G1) are Fe-rich, electron-rich and p-poor, all of which (13/13) are associated with type III bursts, whereas half of them do not present shock signatures (e.g., type II bursts) (6/13). The majority of the events of group $1(10 / 13)$ have a coronal foot point within the "cone of observations" of $\left[-50^{\circ}, 50^{\circ}\right]$ (Fig. 20), consistently with the results of Kallenrode et al. (1992) for impulsive events. Moreover, 7/13 are associated with impulsive SFs, according to the classification by Park et al. (2010). Hence, most of them could be labelled as impulsive SEP events (Cliver 1996). However, events of all other groups are also associated with similar solar events. In addition, almost all SEP events (158/174) had associated CMEs. Although Kahler (2001) reported that impulsive SEP events are associated with relatively narrow CMEs $\left(10^{\circ}-40^{\circ}\right)$ and Cane et al. (2010) found CMEs associated with such events with sizes $\geq 50^{\circ}$, our sample shows that the mean size of the CME for impulsive SEP events is significantly larger (i.e. $\sim 109^{\circ}$ ).

Figure 16 shows that the composition in the low energy range appears to be Fe-rich, with respect to the average SEP value of $\mathrm{Fe} / \mathrm{O}$ (0.134, Reames 1995). Furthermore, several SEP events from groups 3 and 4, which are defined to be p-rich and Fe-poor and in essence are gradual SEP events, seem to be also Fe-rich at low energies. Therefore, besides the Fe-rich material from impulsive SEP events it is possible that CMEdriven shocks accelerate an Fe-rich composition (Mewaldt et al. 2006). Additional Fe might come from ions accelerated by the associated SF that are then re-accelerated by the shock. In addition, there is evidence that larger SEP events often result when a fast CME follows a slower CME (Gopalswamy et al. 2002). Whether this is a result of "CME interactions" (Gopalswamy et al. 2002) or "preconditioning" of the medium by the preceding CME (Kahler \& Vourlidas 2014) is still unclear, but in this situation the shock from the second CME may pass through freshly ejected CME material, which often has highly-ionized $\mathrm{Fe}$ and other anomalous abundances (Mewaldt et al. 2006).

At the same time, it becomes apparent that, although, the $\mathrm{Fe} / \mathrm{O}$ ratios are a useful tool and a clustering of the events as a function of their grouping is evident, a clear-cut distinction of the SEP events (Fig. 16) cannot be achieved based solely on the $\mathrm{Fe} / \mathrm{O}$ ratio.

For the part of our catalogue that covers solar cycle 23 and the ascending phase of solar cycle 24 (1997-2013), 73\% (127/ 174) SEP events are clearly associated with both type III and type II radio bursts. Moreover, for every group of SEP events, the onset time of the identified type III burst was marked prior to the maximum of the SF flux and thus during the impulsive phase of the SF (Figs. 18 and 17), with only a few exceptions. As already discussed in Section 1, there are two basic accelerators: SFs and CME-driven shocks. If the connection between the parent solar source and the observing point does exist, particles shall be routed to and recorded by a particle detector. There is a physical limitation that can lead to the exclusion of particles accelerated by SFs to result in an SEP event, provided that a flare did occur. That is the so-called confinement of the particles in magnetic structures of the low corona (Klein et al. 2005, 2010, 2011; Trottet et al. 2015). Since most of the SEP events in our database were associated with a SF, and the majority of the well-observed events - from solar cycle 23 onwards - is associated with type III radio bursts, it is suggested that the majority of particles accelerated in the SEP associated SF are not confined. However, in certain cases, if no meaningful association with type III bursts could be identified and if the flux of the SEP event was rather low, such confined SFs cannot be excluded. The cone of $\pm 50^{\circ}$ for 
$\Delta \Phi$ which holds events from every group (Fig. 20) is difficult to be reconciled only with a "localized" release of particles. At the same time $87 \%(68 / 78)$ of these events, within the cone, are associated with type II radio bursts and thus support the argument of shock acceleration (Kallenrode et al. 1992). Further work should focus on the effect of $\Delta \Phi$ on the derived SEP flux (Lario et al. 2013), given the fact that, two critical $\Delta \Phi$ at about $30^{\circ}$ and $50^{\circ}$, delimiting three zones for which the importance of the longitudinal gradient is different from one another greatly affect the flux variability of SEP events (Gardini et al. 2011).

The question that arises is if we can classify the events as mixed and/or hybrid, in accordance with the proposition of Kallenrode (2003) and Kocharov \& Torsti (2002) and the expanded classification of SEP events (Cliver 1996). Groups 1 and 2 being Fe-rich, e-rich and p-poor would consist primarily of particles accelerated at SFs, whereas the SEP events of groups $3-5$, which are related to shocks, would be composed primarily of particles accelerated at the CME-driven shock. First, we note that broad limits define the classification of hybrid events using radio diagnostics (e.g., type II-IV radio bursts), the duration of the SFs, abundance ratios (e.g., $\mathrm{Fe} / \mathrm{O}$ ), recordings of electrons, widths of CMEs and the longitudinal cone, in line with the proposed expanded SEP classification system by Cliver 1996 (their Tab. 2 (supplementary online material)). Nevertheless, it was not possible to strictly attribute the events to each of the proposed categories (i.e. pure impulsive, mixed impulsive, mixed gradual, gradual). This is because: [a] all but nine events in our sample was associated with a CME, including $11 / 13$ events of group 1 ; [b] the $\mathrm{Fe} / \mathrm{O}$ ratios are generally high, although, as expected, they demonstrate a clustering at higher values for the events of groups 1 and 2 (which are found to be Fe-rich at both high and low energies), whereas they are lower for the events of groups 4 and 5; albeit, at low energies the Fe-rich character is dominant even for the events of group 4. At the same time, it is evident that most of the SEP events in our catalogue do not conform to a simple two-class paradigm, exhibiting both impulsive phase (type III radio bursts) and shock (type II radio bursts) emissions. As a result, it is not possible to isolate the causative of an SEP event, i.e. SFs and/or CMEs in our database since both tracers of flare and shock acceleration are present. In conclusion, our results point to a continuum of SEP events, following the broad lines of the proposed scheme for hybrid and/or mixed SEP events. However, no clear-cut indication of specific parameters that distinguish the events per sub-category (Cliver 1996) of the hybrid SEP events could be achieved, in line with the proposition of Cane et al. (2010). Nonetheless, Table 2 (supplementary online material) indicates the SEP events attributed to each of the five groups with group 1 being closer by definition to the "pure impulsive" sub-category, followed by group 2 and group 3 (e.g., "mixed impulsive" and "mixed gradual", respectively) and finally group 4 and group 5 that are closest to the sub-category of "gradual" SEP events.

\subsection{Statistical analysis}

\section{- Energy-dependent contributions of SFs and CMEs in SEP events}

We showed that the proton peak flux and fluence of the SEP events vary as a function of both SF and CME properties, examined in this work. However, the correlations range from low to moderate, with the significant ones marked for the speed of the CME, the magnitude and the fluence of the SF (see Tab. 2 (supplementary online material)). In particular, the correlation between the velocity of the $\mathrm{CME}$ and the proton peak flux appears to decrease with the increasing integrated particle energy (column 8 of Tab. 2 (supplementary online material)), but at the same time the correlation of the logarithm of the SXR peak flux to the proton peak flux appears to remain relatively stable $(\approx 0.47)$ from $E>10$ to $E>100 \mathrm{MeV}$. Additionally, for $E>10 \mathrm{MeV}$ the correlation between the proton peak flux and the CME velocity seems to be stronger (0.57) compared to the correlation with the logarithm of the SXR peak flux (0.49), with a reverse situation being present at higher energies (for $E>100 \mathrm{MeV}$ the proton peak flux presents a correlation coefficient of 0.40 with the $\mathrm{CME}$ velocity and of 0.49 with the logarithm of the SXR peak flux). The transition is spotted for $E>60 \mathrm{MeV}$ (see Tab. 2 (supplementary online material)). Furthermore, the correlation of the proton peak flux to the fluence of the flare seems to be fairly stable, similar to the obtained correlation of the logarithm of the SXR peak flux. This is also the outcome obtained from the correlations with the fluence of the SEP event. Nonetheless, the correlation coefficients for the fluence of the flare seem to drop with respect to the increasing integrated particle energy (column 2 of Tab. 2 (supplementary online material)), with the transition spotted at $E>60 \mathrm{MeV}$. These results possibly suggest that at low energies, CMEs have a dominant role, not excluding, also, the contribution of particles accelerated by SFs (Cane et al. 2002; Dierckxsens et al. 2015; Trottet et al. 2015). The former result is apparent in Figures 12A and $12 \mathrm{~B}$, which illustrate the higher correlation of the $\mathrm{CME}$ speed to the obtained proton peak fluxes at $E>10$ and $E>30 \mathrm{MeV}$ compared to the achieved correlation for higher energies (Figs. 12C and 12D). At the same time, gradual $S E P$ events (group 4 and group 5) present evidence of a coronal propagation within $\pm 50^{\circ}$ (Fig. 20) and are associated with type III radio bursts (Fig. 18). Therefore, the possibility that SF acceleration is, also, involved in these events, together with the dominant acceleration by CMEs, cannot be excluded.

\section{- Factors relevant to significant radiation risk}

The mapping of the logarithm of the proton peak flux at $E>10 \mathrm{MeV}$ on different pairs of parent solar source characteristics clearly illustrated its dependence on strong solar flares and fast CMEs that result in enhanced radiation storms, as opposite to weak solar flares and slow CMEs. It was shown that enhanced proton peak fluxes (e.g., $\geq \mathrm{S} 3$ ) are present over a broad longitudinal distribution for SFs $\left(-70\right.$ to $\left.70^{\circ}\right)$. It was also shown that SFs that last $\geq 60 \mathrm{~min}$ are also associated with strong radiation storms $(\geq \mathrm{S} 3)$ even for SFs with a magnitude of M5.0, while all $\geq X 1.0$ SFs lasting up to $200 \mathrm{~min}$ lead to enhanced radiation levels $(\geq S 2)$. These findings are also consistent with those obtained by investigating the fluence of the SEP events in our catalogue. It was demonstrated that significant radiation risk emerges under three basic conditions: (a) strong SFs ( $\geq$ X5.0 lasting even less than $60 \mathrm{~min}$ or $\geq$ M5.0 lasting more than $60 \mathrm{~min}$ ) (Fig. 21C); (b) significant CME speed (Fig. 21D) and (c) events whose source is situated on the central part of the visible solar disk (Fig. 15). Conditions (b), (c) and the second part of condition (a) are consistent with the scenario of a fast CME-driven shock that continuously feeds the magnetic field lines connecting the shock to the observer with accelerated particles (interplanetary shock 
dominated SEP events). These factors signify the important role of SEP events associated with central solar sources. In conclusion, it is noteworthy that this mapping helps to understand how does the logarithm of the proton peak flux at $E>10 \mathrm{MeV}$ (a crucial SEP metric) change as a function of different pairs of variables defined by its parent solar events and at the same time quantifies the combined effect of the variables upon the expected proton peak flux.

Acknowledgements. Part of this work was supported through the ESA Contract no. 4000109641/13/NL/AK "Improvement of Solar Particle Events and Flare Prediction" and the "SPECS: Solar Particle Events and foreCasting Studies" research grant of the National Observatory of Athens. Furthermore, this work was also performed in the framework of PROTEAS project within GSRTs KRIPIS action, funded by Greece and the European Regional Development Fund of the European Union under the O.P. Competitiveness and Entrepreneurship, NSRF 2007-2013 and the Regional Operational Program of Attica. The authors would like to thank the SEPEM Team for the provision of the cleaned GOES data and to further acknowledge Angels Aran (University of Barcelona, Spain) for the provision of her SEP catalogue, based on SEPEM. AP would also like to gratefully acknowledge the guidance of Karl-Ludwig Klein, Anatoly Belov and Eugenia Eroshenko concerning data sources prior to 1997 and the technical advice of John Dimitroulakos. The authors would further like to thank Bernd Heber and another anonymous referee for a critical and constructive reading of the manuscript, as well as Rami Vainio for valuable comments that greatly improved the context of the paper. The research leading to these results has utilized SEPServer, a European research Project that received funding from the European Community's Seventh Framework Programme (FP7/ 2007-2013) under Grant Agreement no. 262773. The CME Catalog used in this work is generated and maintained at the CDAW Data Center by NASA and The Catholic University of America in cooperation with the Naval Research Laboratory. SOHO is a project of international cooperation between ESA and NASA. The use of the online SMM catalogues is courtesy of $\mathrm{HAO} / \mathrm{SMM} \mathrm{C} / \mathrm{P}$ project team and NASA. HAO is a division of the National Center for Atmospheric Research, which is supported by the National Science Foundation. The use of the MLSO CME data archives is courtesy of the Mauna Loa Solar Observatory, operated by the High Altitude Observatory, as part of the National Center for Atmospheric Research (NCAR). NCAR is supported by the National Science Foundation. The editor thanks Bernd Heber and another anonymous referee for their assistance in evaluating this paper.

\section{References}

Agueda, N., K.-L. Klein, N. Vilmer, R. Rodríguez-Gasén, O. Malandraki, et al. Release timescales of solar energetic particles in the low corona. $A \& A, \mathbf{5 7 0}, \mathrm{A} 5,2014$, DOI: $10.1051 / 0004-6361 / 201423549$.

Anastasiadis, A. Acceleration of solar energetic particles: the case of solar flares. J. Atmos. Sol. Terr. Phys., 64(5), 481-488, 2002, DOI: $10.1051 / 0004-6361 / 201423549$.

Aschwanden, M.J. Particle acceleration and kinematics in solar flares - a synthesis of recent observations and theoretical concepts (Invited Review). Space Sci. Rev., 101(1-2), 1-227, 2002, DOI: $10.1023 / \mathrm{A}: 1019712124366$.

Balch, C.C. SEC proton prediction model: verification and analysis. Radiat. Meas., 30(3), 231-250, 1999,

DOI: $10.1016 / \mathrm{S} 1350-4487(99) 00052-9$.

Balch, C.C. Updated verification of the Space Weather Prediction Center's solar energetic particle prediction model. Space Weather, 6, S01001, 1-13, 2008, DOI: 10.1029/2007SW000337.

Belov, A., H. Garcia, V. Kurt, H. Mavromichalaki, and M. Gerontidou. Proton enhancements and their relation to the
X-ray flares during the three last solar cycles. Sol. Phys., 229 (1), 135-159, 2005, DOI: 10.1007/s11207-005-4721-3.

Benz, A.O. Flare observations. Living Rev. Sol. Phys., 5 (1), 1-64, 2008, DOI: $10.12942 /$ lrsp-2008-1.

Bougeret, J.-L., M. Kaiser, P. Kellogg, R. Manning, K. Goetz, et al. Waves: The radio and plasma wave investigation on the Wind spacecraft. Space Sci. Rev., 71 (1-4), 231-263, 1995, DOI: $10.1007 / \mathrm{BF} 00751331$.

Bougeret, J.-L., K. Goetz, M. Kaiser, S. Bale, P. Kellogg, et al. S/ WAVES: The radio and plasma wave investigation on the STEREO mission. Space Sci. Rev., 136 (1-4), 487-528, 2008, DOI: $10.1007 / \mathrm{s} 11214-007-9298-8$.

Brueckner, G., R. Howard, M. Koomen, C. Korendyke, D. Michels, et al. The large angle spectroscopic coronagraph (LASCO). Sol. Phys., 162 (1-2), 357-402, 1995, DOI: 10.1007/BF00733434.

Cane, H., and D. Lario. An introduction to CMEs and energetic particles. Space Sci. Rev., 123 (1-3), 45-56, 2006, DOI: $10.1007 / \mathrm{s} 11214-006-9011-3$.

Cane, H.V., W. Erickson, and N. Prestage. Solar flares, type III radio bursts, coronal mass ejections, and energetic particles. J. Geophys. Res. [Space Phys.], 107 (A10), SSH-14, 2002, DOI: 10.1029/2001JA000320.

Cane, H. V., I. G. Richardson, and T. T. von Rosenvinge (2010), A study of solar energetic particle events of 1997-2006: Their composition and associations, J. Geophys. Res., 115, A08101, 1-18.

Cliver, E. Solar flare gamma-ray emission and energetic particles in space. In: High energy solar physics, vol. 374, AIP Publishing, Melville, New York, pp. 45-60, 1996, DOI: 10.1063/1.50980.

Colaninno, R.C., and A. Vourlidas. First determination of the true mass of coronal mass ejections: a novel approach to using the two STEREO viewpoints. Astrophys. J., 698 (1), 852, 2009,

URL http://stacks.iop.org/0004-637X/698/i=1/a=852.

Crosby, N., D. Heynderickx, P. Jiggens, A. Aran, and B. Sanahuja. SEPEM: a tool for statistical modelling the solar energetic particle environment. Space Weather, 13, 406-426, 2015, DOI: 10.1002/2013SW001008.

Dennis, B.R., and D.M. Zarro. The Neupert effect: what can it tell us about the impulsive and gradual phases of solar flares? Sol. Phys., 146 (1), 177-190, 1993, DOI: 10.1007/BF00662178.

Dierckxsens, M., K. Tziotziou, S. Dalla, I. Patsou, M. Marsh, N. Crosby, O. Malandraki, and G. Tsiropoula. Relationship between solar energetic particles and properties of flares and CMEs: statistical analysis of solar cycle 23 events. Sol. Phys., 290 (3), 841-874, 2015, DOI: 10.1007/s11207-014-0641-4.

Dougherty, B.L., H. Zirin, and K. Hsu. Statistical correlations between solar microwave bursts and coronal mass ejections. Astrophys. J., 577 (1), 457, 2002, DOI: 10.1086/342162.

Dresing, N., R. Gómez-Herrero, A. Klassen, B. Heber, Y. Kartavykh, and W. Dröge. The large longitudinal spread of solar energetic particles during the 17 January 2010 solar event. Sol. Phys., 281 (1), 281-300, 2012, DOI: 10.1007/s11207-012-0049-y.

Dröge, W., Y. Kartavykh, N. Dresing, B. Heber, and A. Klassen. Wide longitudinal distribution of interplanetary electrons following the 7 February 2010 solar event: observations and transport modeling. J. Geophys. Res. [Space Phys.], 119 (8), 6074-6094, 2014, DOI: 10.1002/2014JA019933.

Fenton, N., and M. Neil. Risk assessment and decision analysis with Bayesian networks, CRC Press/Taylor \& Francis Group, Boca Raton/London/New York, 2012.

Fisher, R. On the probable error of a coefficient of correlation deduced from a small sample. Metron, 1, 3-32, 1921.

Garcia, H.A. Forecasting methods for occurrence and magnitude of proton storms with solar hard X rays. Space Weather, 2, S06003, 1-10, 2004a, DOI: 10.1029/2003SW000035.

Garcia, H.A. Forecasting methods for occurrence and magnitude of proton storms with solar soft X rays. Space Weather, 2, S02002, 1-10, 2004b, DOI: 10.1029/2003SW000001.

Gardini, A., M. Laurenza, and M. Storini. SEP events and multispacecraft observations: constraints on theory. Adv. Space Res., 47 (12), 2127-2139, 2011, DOI: 10.1016/j.asr.2011.01.025. 
Georgoulis, M.K. Magnetic complexity in eruptive solar active regions and associated eruption parameters. Geophys. Res. Lett., 35, L06S02, 1-5, 2008, DOI: 10.1029/2007GL032040.

Gold, R., S. Krimigis, S. Hawkins III, D. Haggerty, D. Lohr, E. Fiore, T. Armstrong, G. Holland, and L. Lanzerotti. Electron, proton, and alpha monitor on the advanced composition explorer spacecraft. Space Sci. Rev., 86 (1-4), 541-562, 1998, DOI: $10.1023 / \mathrm{A}: 1005088115759$.

Gopalswamy, N. Energetic particle and other space weather events of solar cycle 24. AIP Conference Proceedings, 1500, 14-19, 2012, DOI: $10.1063 / 1.4768738$.

Gopalswamy, N., S. Yashiro, G. Michałek, M. Kaiser,R. Howard, D. Reames, R. Leske, and T. Von Rosenvinge. Interacting coronal mass ejections and solar energetic particles. Astrophys. J. Lett., 572 (1), L103, 2002, DOI: 10.1086/341601.

Gopalswamy, N., S. Yashiro, A. Lara, M. Kaiser, B.J. Thompson, P.T. Gallagher, and R.A. Howard. Large solar energetic particle events of cycle 23: a global view. Geophys. Res. Lett., 30 (12), 8015-8018, 2003, DOI: 10.1029/2002GL016435.

Gopalswamy, N., S. Yashiro, S. Krucker, G. Stenborg, and R.A. Howard. Intensity variation of large solar energetic particle events associated with coronal mass ejections. J. Geophys. Res. [Space Phys.], 109, A12105, 1-18, 2004,

DOI: $10.1029 / 2004 J A 010602$.

Gopalswamy, N., S. Yashiro, G. Michalek, G. Stenborg, A. Vourlidas, S. Freeland, and R. Howard. The SOHO/LASCO CME catalog. Earth Moon Planet, 104, 295-313, 2009, DOI: $10.1007 / \mathrm{s} 11038-008-9282-7$.

Kahler, S.W. The role of the big flare syndrome in correlations of solar energetic proton fluxes and associated microwave burst parameters. J. Geophys. Res. [Space Phys.], 87 (A5), 3439-3448, 1982, DOI: 10.1029/JA087iA05p03439.

Kahler, S.W. The correlation between solar energetic particle peak intensities and speeds of coronal mass ejections: effects of ambient particle intensities and energy spectra. J. Geophys. Res. [Space Phys.], 106 (A10), 20947-20955, 2001, DOI: 10.1029/2000JA002231.

Kahler, S.W. Characteristic times of gradual solar energetic particle events and their dependence on associated coronal mass ejection properties. Astrophys. J., 628 (2), 1014, 2005, DOI: $10.1086 / 431194$.

Kahler, S.W., and A. Vourlidas. A comparison of the intensities and energies of gradual solar energetic particle events with the dynamical properties of associated coronal mass ejections. Astrophys. J., 769 (2), 143, 2013, DOI: $10.1088 / 0004-637 X / 769 / 2 / 143$.

Kahler, S.W., and A. Vourlidas. Do interacting coronal mass ejections play a role in solar energetic particle events? Astrophys. $J ., 784$ (1), 47, 2014, DOI: 10.1088/0004-637X/784/1/47.

Kallenrode, M.-B. Current views on impulsive and gradual solar energetic particle events. J. Phys. G: Nucl. Part. Phys., 29 (5), 965, 2003, DOI: 10.1088/0954-3899/29/5/316.

Kallenrode, M.-B., E. Cliver, and G. Wibberenz. Composition and azimuthal spread of solar energetic particles from impulsive and gradual flares. Astrophys. J., 391, 370-379, 1992.

Klein, K.-L., and A. Posner. The onset of solar energetic particle events: prompt release of deka-MeV protons and associated coronal activity. $A \& A, \mathbf{4 3 8}(\mathbf{3}), 1029-1042,2005$, DOI: $10.1051 / 0004-6361: 20042607$.

Klein, K.-L., S. Krucker, G. Trottet, and S. Hoang. Coronal phenomena at the release of solar energetic electron events. $A \& A$, 431 (3), 1047-1060, 2005, DOI: 10.1051/0004-6361:20041258.

Klein, K.-L., G. Trottet, and A. Klassen. Energetic particle acceleration and propagation in strong CME-less flares. Sol. Phys., 263 (1-2), 185-208, 2010, DOI: $10.1007 / \mathrm{s} 11207-010-9540-5$.

Klein, K.-L., G. Trottet, S. Samwel, and O. Malandraki. Particle acceleration and propagation in strong flares without major solar energetic particle events. Sol. Phys., 269 (2), 309-333, 2011, DOI: $10.1007 / \mathrm{s} 11207-011-9710-0$.
Kocharov, L., and J. Torsti. Hybrid solar energetic particle events observed on board SOHO. Sol. Phys., 207 (1), 149-157, 2002, DOI: 10.1023/A:1015540311183.

Kouloumvakos, A., A. Nindos, E. Valtonen, C.E. Alissandrakis, O. Malandraki, P. Tsitsipis, A. Kontogeorgos, X. Moussas, and A. Hillaris. Properties of solar energetic particle events inferred from their associated radio emission. $A \& A, \mathbf{5 8 0}$, A80, 2015, DOI: 10.1051/0004-6361/201424397.

Kurt, V., A. Belov, H. Mavromichalaki, and M. Gerontidou. Statistical analysis of solar proton events. Ann. Geophys., 22 (6), 2255-2271, 2004, DOI: 10.5194/angeo-22-2255-2004.

Lario, D., A. Aran, R. Gómez-Herrero, N. Dresing, B. Heber, G. Ho, R. Decker, and E. Roelof. Longitudinal and radial dependence of solar energetic particle peak intensities: STEREO, ACE, SOHO, GOES, and MESSENGER observations. Astrophys. J., 767 (1), 41, 2013, DOI: 10.1088/0004-637X/767/1/41.

Lario, D., N. Raouafi, R.-Y. Kwon, J. Zhang, R. Gómez-Herrero, N. Dresing, and P. Riley. The solar energetic particle event on 2013 April 11: an investigation of its solar origin and longitudinal spread. Astrophys. J., 797 (1), 8, 2014, DOI: $10.1088 / 0004-637 X / 797 / 1 / 8$.

Lario, D., R.-Y. Kwon, A. Vourlidas, N. Raouafi, and D. Haggerty. Longitudinal properties of a widespread solar energetic particle event on 2014 February 25: evolution of the associated CME shock. Astrophys. J., 819 (1), 72, 2016 DOI: $10.3847 / 0004-637 \mathrm{X} / 819 / 1 / 72$.

Laurenza, M., E. Cliver, J. Hewitt, M. Storini, A. Ling,C. Balch, and M. Kaiser. A technique for shortterm warning of solar energetic particle events based on flare location, flare size, and evidence of particle escape. Space Weather, 7 (4), 2009, DOI: 10.1029/2007SW000379.

Lintunen, J., and R. Vainio. Solar energetic particle event onset as analyzed from simulated data. $A \& A, 420$ (1), 343-350, 2004, DOI: 10.1051/0004-6361:20034247.

Mason, G., R. Gold, S. Krimigis, J. Mazur, G. Andrews, et al. The ultra-low-energy isotope spectrometer (ULEIS) for the ACE spacecraft. In: The advanced composition explorer mission, Springer Science+Business Media B.V., Dordrecht, Netherlands, pp. 409-448, 1998, DOI: 10.1007/978-94-011-4762-0_16.

Maunder, E.W. Note on the distribution of sun-spots in heliographic latitude, 1874-1902. Mon. Not. R. Astron. Soc., 64, 747-761, 1904.

McComas, D., S. Bame, P. Barker, W. Feldman, J. Phillips, P. Riley, and J. Griffee. Solar wind electron proton alpha monitor (SWEPAM) for the Advanced Composition Explorer. In: The advanced composition explorer mission, Springer Science+ Business Media B.V., Dordrecht, Netherlands, 563-612, 1998, DOI: 10.1007/978-94-011-4762-0_20.

Mewaldt, R., C. Cohen, and G. Mason. The source material for large solar energetic particle events. In:N. Gopalswamy, R. Mewaldt, and J. Torsti, Editors. Solar Eruptions and Energetic Particles, Wiley Online Library, 115-125, 2006, DOI: 10.1029/165GM12.

Neupert, W.M. Comparison of solar X-ray line emission with microwave emission during flares. Astrophys. J., 153, L59, 1968.

Nolte, J., and E. Roelof. Large-scale structure of the interplanetary medium. Sol. Phys., 33 (2), 483-504, 1973, DOI: $10.1007 / \mathrm{BF} 00152435$.

Núñez, M. Predicting solar energetic proton events $(E>10 \mathrm{MeV})$. Space Weather, 9, S07003, 1-28, 2011, DOI: $10.1029 / 2010$ SW000640.

Onsager, T., R. Grubb, J. Kunches, L. Matheson, D. Speich, R.W. Zwickl, and H. Sauer. Operational uses of the GOES energetic particle detectors. In:SPIE's 1996 International Symposium on Optical Science, Engineering, and Instrumentation, International Society for Optics and Photonics, Bellingham, Washington, pp. 281-290, 1996, DOI: 10.1117/12.254075.

Owens, M., and P. Cargill. Predictions of the arrival time of Coronal Mass Ejections at 1AU: an analysis of the causes of errors. Ann. Geophys., 22 (2), 661-671, 2004, DOI: 10.5194/angeo-22-661-2004 
Papaioannou, A., O. Malandraki, N. Dresing, B. Heber, and K.-L. Klein. SEPServer catalogues of solar energetic particle events at 1 AU based on STEREO recordings: 2007-2012. A\&A, 569, A96, 2014a, DOI: 10.1051/0004-6361/201323336.

Papaioannou, A., G. Souvatzoglou, P. Paschalis, M. Gerontidou, and H. Mavromichalaki. The first ground-level enhancement of solar cycle 24 on 17 May 2012 and its real-time detection. Sol. Phys., 289 (1), 423-436, 2014b, DOI: 10.1007/s11207-013-0336-2.

Papaioannou, A., A. Anastasiadis, I. Sandberg, M. Georgoulis, G. Tsiropoula, K. Tziotziou, P. Jiggens, and A. Hilgers. A novel forecasting system for solar particle events and flares (FORSPEF). J. Phys: Conf. Ser., 632 (1), 012075, 2015, DOI: $10.1088 / 1742-6596 / 632 / 1 / 012075$.

Park, J., Y.-J. Moon, D. Lee, and S. Youn. Dependence of solar proton events on their associated activities: Flare parameters. J. Geophys. Res. [Space Phys.], 115, A10105, 1-6, 2010, DOI: 10.1029/2010JA015330.

Park, J., Y.-J. Moon, and N. Gopalswamy. Dependence of solar proton events on their associated activities: coronal mass ejection parameters. J. Geophys. Res. [Space Phys.], 117, A08108, 1-7, 2012, DOI: $10.1029 / 2011$ JA017477.

Parker, E. Dynamical theory of the solar wind. Space Sci. Rev., 4 (5-6), 666-708, 1965, DOI: 10.1007/BF00216273.

Pick, M., and N. Vilmer. Sixty-five years of solar radioastronomy: flares, coronal mass ejections and Sun - Earth connection. Astron. Astrophys. Rev., 16 (1), 1-153, 2008, DOI: $10.1007 / \mathrm{s} 00159-008-0013-x$.

Posner, A. Up to 1-hour forecasting of radiation hazards from solar energetic ion events with relativistic electrons. Space Weather, 5, S05001, 1-28, 2007, DOI: 10.1029/2006SW000268.

Reames, D. Coronal abundances determined from energetic particles. Adv. Space Res., 15 (7), 41-51, 1995, DOI: $10.1016 / 0273-1177(94) 00018-\mathrm{V}$.

Reames, D.V. Particle acceleration at the Sun and in the heliosphere. Space Sci. Rev., 90 (3-4), 413-491, 1999 ,

DOI: $10.1023 / \mathrm{A}: 1005105831781$

Reames, D.V. Magnetic topology of impulsive and gradual solar energetic particle events. Astrophys. J. Lett., 571 (1), L63, 2002, DOI: $10.1086 / 341149$.

Reames, D.V. The two sources of solar energetic particles. Space Sci. Rev., 175 (1-4), 53-92, 2013, DOI: $10.1007 / \mathrm{s} 11214-013-9958-9$.

Reames, D.V. What are the sources of solar energetic particles? Element abundances and source plasma temperatures. Space Sci. Rev., 194 (1-4), 303-327, 2015, DOI: $10.1007 / \mathrm{s} 11214-015-0210-7$.

Reid, H.A.S., and H. Ratcliffe. A review of solar type III radio bursts. Res. Astron. Astrophys., 14 (7), 773, 2014, DOI: $10.1088 / 1674-4527 / 14 / 7 / 003$.

Richardson, I., T. von Rosenvinge, H. Cane, E. Christian, C. Cohen, et al. $>25 \mathrm{MeV}$ proton events observed by the high energy telescopes on the STEREO A and B spacecraft and/or at Earth during the first $\approx$ seven years of the STEREO Mission. Sol. Phys., 289 (8), 3059-3107, 2014, DOI: $10.1007 / \mathrm{s} 11207-014-0524-8$.

Rodriguez, J., J. Krosschell, and J. Green. Intercalibration of GOES 8-15 solar proton detectors. Space Weather, 12 (1), 92-109, 2014, DOI: 10.1002/2013SW000996.

Rotter, T., A. Veronig, M. Temmer, and B. Vršnak. Real-time solar wind prediction based on SDO/AIA coronal hole data. Sol. Phys., 290 (5), 1355-1370, 2015, DOI: 10.1007/s11207-015-0680-5.

Rouillard, A., N. Sheeley, A. Tylka, A. Vourlidas, C. Ng, et al. The longitudinal properties of a solar energetic particle event investigated using modern solar imaging. Astrophys. J., 752 (1), 44, 2012, DOI: 10.1088/0004-637X/752/1/44.
Sáiz, A., P. Evenson, D. Ruffolo, and J.W. Bieber. On the estimation of solar energetic particle injection timing from onset times near Earth. Astrophys. J., 626 (2), 1131, 2005, DOI: 10.1086/430293.

Sandberg, I., P. Jiggens, D. Heynderickx, and I. Daglis. Cross calibration of NOAA GOES solar proton detectors using corrected NASA IMP-8/GME data. Geophys. Res. Lett., 41 (13), 4435-4441, 2014, DOI: 10.1002/2014GL060469.

Sheeley, N., J. Walters, Y.-M. Wang, and R. Howard. Continuous tracking of coronal outflows: two kinds of coronal mass ejections. J. Geophys. Res. [Space Phys.], 104 (A11), 24739-24767, 1999, DOI: 10.1029/1999JA900308.

Shen, D., and Z. Lu. Computation of correlation coefficient and its confidence interval in SAS. SUGI: Paper 170-31, SUGI 31 Proceedings, March 26-29,San Francisco, CA, 2006.

Smart, D., and M. Shea. PPS-87: a new event oriented solar proton prediction model. Adv. Space Res., 9 (10), 281-284, 1989, DOI: 10.1016/0273-1177(89)90450-X.

Smart, D., M. Shea, H.E. Spence, and L. Kepko. Two groups of extremely large $>30 \mathrm{MeV}$ solar proton fluence events. $A d v$. Space Res., 37 (9), 1734-1740, 2006, DOI: 10.1016/j.asr.2005.09.008.

Stone, E., A. Frandsen, R. Mewaldt, E. Christian, D. Margolies, J. Ormes, and F. Snow. The advanced composition explorer, Springer Science+Business Media B.V., Dordrecht, Netherlands, pp. 1-22, 1998a, DOI: 10.1007/978-94-011-4762-01.

Stone, E.C., C. Cohen, W. Cook, A. Cummings, and B. Gauld. The solar isotope spectrometer for the advanced composition explorer. In: The advanced composition explorer mission, Springer Science+Business Media B.V., Dordrecht, Netherlands, pp. 357-408, 1998b, DOI: 10.1007/978-94-011-4762-015.

Trottet, G., S. Samwel, K.-L. Klein, T.D. de Wit, and R. Miteva. Statistical evidence for contributions of flares and coronal mass ejections to major solar energetic particle events. Sol. Phys., 290 (3), 819-839, 2015

Unzicker, A., and R.F. Donnelly. Calibration of X-ray ion chambers for the Space Environment Monitoring System. Technical report COM-75-10667. In: National Oceanic and Atmospheric Administration, Boulder, Colo. (USA). Space Environment Lab, 1974.

Vainio, R., N. Agueda, A. Aran, and D. Lario. Modeling of solar energetic particles in interplanetary space. In: J. Lilensten, Editor. Space weather: research towards applications in Europe, Springer, Dordrecht, The Netherlands, 27-37, ISBN: 978-14020-5446-4, 2007, DOI: 10.1007/1-4020-5446-74.

Vainio, R., L. Desorgher, D. Heynderickx, M. Storini, E. Flückiger, et al. Dynamics of the Earths particle radiation environment. Space Sci. Rev., 147 (3-4), 187-231, 2009, DOI: $10.1007 / \mathrm{s} 11214-009-9496-7$.

Vainio, R., E. Valtonen, B. Heber, O.E. Malandraki, A. Papaioannou, et al. The first SEPServer event catalogue $\sim 68-\mathrm{MeV}$ solar proton events observed at 1 AU in 1996-2010. J. Space Weather Space Clim., 3, A12, 2013, DOI: 10.1051/swsc/2013030.

Vršnak, B., D. Ruždjak, D. Sudar, and N. Gopalswamy. Kinematics of coronal mass ejections between 2 and 30 solar radii. $A \& A$, 423 (2), 717-728, 2004, DOI: 10.1051/0004-6361:20047169.

Vršnak, B., D. Sudar, and D. Ruždjak. The CME-flare relationship: are there really two types of CMEs? $A \& A, 435$ (3), 1149-1157, 2005, DOI: 10.1051/0004-6361:20042166.

Wiedenbeck, M., G. Mason, C. Cohen, N. Nitta, R. Gómez-Herrero, and D. Haggerty. Observations of solar energetic particles from 3 He-rich events over a wide range of heliographic longitude. Astrophys. J., 762 (1), 54, 2012, DOI: $10.1088 / 0004-637 \mathrm{X} / 762 / 1 / 54$.

Youssef, M. On the relation between the CMEs and the solar flares. NRIAG Journal of Astronomy and Geophysics, 1, 172-178, 2012, DOI: 10.1016/j.nrjag.2012.12.014.

Cite this article as: Papaioannou A, Sandberg I, Anastasiadis A, Kouloumvakos A, Georgoulis MK, et al. Solar flares, coronal mass ejections and solar energetic particle event characteristics. J. Space Weather Space Clim., 6, A42, 2016, DOI: 10.1051/swsc/2016035. 